\title{
INVARIANT EIGENDISTRIBUTIONS ON A SEMISIMPLE LIE GROUP
}

\author{
BY \\ HARISH-CHANDRA
}

1. Introduction. Let $G$ be a connected semisimple Lie group and 3 the algebra of all differential operators on $G$ which commute with both left and right translations of $G$. One of the main objects of this paper is to show that every invariant eigendistribution $T$ of 3 on $G$, is actually a locally summable function $F$ which is analytic on the regular set $G^{\prime}$ of $G$ (Theorem 2). In particular, this implies that the character of an irreducible unitary representation of $G$ is a function.

In the second part we examine the behavior of $F$ around the singular points of $G$ (see $\S \S 19,20)$. This is done by applying the results of $[4(\mathrm{n}), \S \S 8,9]$. The third part is devoted to the detailed study of an invariant integral on $G$, which had been introduced in [4(h), Theorem 2]. Here we have to make use of [4(m), Theorem 1]. The full significance of Theorem 3 for harmonic analysis on $G$ will appear only in later papers. Roughly speaking, it is the group analogue of $[4(\mathrm{~g})$, Theorem 3].

Our methods are substantially the same as those introduced in [4(1)] and [4(n)], although they have now to be applied to the group $G$ instead of its Lie algebra g. Here Theorem 2 of [4(1)] gets replaced by Lemma 22, which is based on Theorem 1 and this, in its turn, depends on Theorem 5 of [4(n)]. The results of $\$ 3$ enable us to limit ourselves to the semisimple points of $G$ and the reduction procedure, outlined above, can be applied to any such point $a$ provided it does not lie in the center $Z$ of $G$. However, if $a \in Z$, the translation by $a^{-1}$ reduces the problem to the case $a=1$. Then we use the results of $\S 14$ to transform it, by means of the exponential mapping, into an analogous question on $\mathfrak{g}$ around zero, which has already been discussed in [4(n)]. This general pattern of proof applies to most results of this paper (e.g., Theorems 1 and 2). However, sometimes it is more convenient to reduce the problem around $a$ directly to the corresponding question around zero on the centralizer $z$ of $a$ in $g$ (see, for example, the proofs of Lemmas 31, 35, 37 and 40).

Theorem 3 is proved by making use of the elementary solution of a certain elliptic "Laplacian" and imitating the argument of [4(g), pp. 208-211]. The Appendix contains a few simple lemmas which are needed in the proof of this theorem.

Received by the editors June 2, 1964. 
This work was partially supported by grants from the Sloan and the National Science Foundations.

2. The mapping $\Gamma_{x}$. Let $G$ be a Lie group, $g$ its Lie algebra over $R$ and $\mathfrak{G}$ the universal enveloping algebra of $\mathfrak{g}_{c}$. For any $X \in \mathfrak{g}_{c}$, let $L_{X}$ and $R_{X}$, respectively, denote the endomorphisms $g \rightarrow X g$ and $g \rightarrow g X(g \in(5)$ of (5. Fix $x \in G$ and define $\left({ }^{1}\right)$ (cf. [4(e), p. 114])

$$
\sigma_{x}(X)=L_{x-1 X}-R_{X} \quad\left(X \in \mathfrak{g}_{c}\right) .
$$

Note that $L_{X}$ and $R_{Y}$ commute and $\left({ }^{2}\right)$

$$
\left[L_{X}, L_{Y}\right]=L_{[X, Y]}, \quad\left[R_{X}, R_{Y}\right]=-R_{[X, Y]}
$$

for $X, Y \in \mathfrak{g}_{c}$. Hence it follows immediately that $\sigma_{x}$ is a representation of $\mathfrak{g}_{c}$ on $\mathfrak{b}$. It may, therefore, be extended (uniquely) to a representation of $(\mathfrak{b}$ which we shall again denote by $\sigma_{x}$. Let $\Gamma_{x}$ denote the linear mapping of $\mathfrak{G} \otimes(\mathfrak{b}$ into (5 such that

$$
\Gamma_{x}\left(g_{1} \otimes g_{2}\right)=\sigma_{x}\left(g_{1}\right) g_{2} \quad\left(g_{1}, g_{2} \in(\mathfrak{F}) .\right.
$$

Let $\lambda$ denote the canonical mapping (see [4(b), p. 192]) of $S\left(\mathfrak{g}_{c}\right)$ onto $(\mathfrak{b}$. We say that an element $g \in \mathfrak{G}$ is homogeneous of degree $d$ if $g \in\left(\mathfrak{F}_{d}=\lambda\left(S_{d}\left(\mathfrak{g}_{c}\right)\right)\right.$ in the notation of $[4(\mathrm{k}), \S 6]$. Put

$$
{ }_{d} \mathfrak{G}=\sum_{0 \leqq m \leqq d} \mathfrak{G}_{m} .
$$

Then it is obvious that $\Gamma_{x}$ defines a linear mapping of $\mathfrak{F}_{d_{1}} \otimes \mathfrak{F}_{d_{2}}$ into $d_{d_{1}+d_{2}}(\mathfrak{F}$.

Let $x \rightarrow x^{a}(x \in G)$ be an automorphism of $G$. Then it defines an automorphism of $\mathfrak{g}$ which can be extended uniquely to an automorphism $g \rightarrow g^{a}(g \in \mathfrak{G})$ of $g$.

LEMMA 1. For any $x \in G$ and $g_{1}, g_{2} \in \mathbb{F}$,

$$
\Gamma_{x^{a}}\left(g_{1}^{a} \otimes g_{2}^{a}\right)=\left(\Gamma_{x}\left(g_{1} \otimes g_{2}\right)\right)^{a} .
$$

Let $A$ denote the automorphism $g \rightarrow g^{a}$ of $(5$. Then one verifies from the definitions that

$$
\sigma_{x^{a}}\left(X^{a}\right)=A \sigma_{x}(X) A^{-1}
$$

for $X \in \mathfrak{g}$. Our assertion is an immediate consequence of this fact.

Lemma 2. Suppose $X_{i}$ and $Y_{j}(1 \leqq i \leqq r, 1 \leqq j \leqq s)$ are elements in $g_{c}$. Fix $x \in G$ and put $X_{i}^{\prime}=x^{-1} X_{i}-X_{i}(1 \leqq i \leqq r)$. Then

$\Gamma_{x}\left(\lambda\left(X_{1} X_{2} \cdots X_{r}\right) \otimes \lambda\left(Y_{1} Y_{2} \cdots Y_{s}\right)\right) \equiv \lambda\left(X_{1}^{\prime} X_{2}^{\prime} \cdots X_{r}^{\prime} Y_{1} Y_{2} \cdots Y_{s}\right) \bmod _{(r+s-1)}(5$.

It follows by an easy induction on $r$ that

(1) As usual $x X=X^{x}=\operatorname{Ad}(x) X$ for $x \in G$ and $X \in \mathfrak{g}_{\mathrm{c}}$.

(2) $[A, B]=A B-B A$ for two endomorphisms $A$ and $B$ of a vector space. 


$$
\Gamma_{x}\left(X_{1} X_{2} \cdots X_{r} \otimes Y_{1} Y_{2} \cdots Y_{s}\right) \equiv X_{1}^{\prime} X_{2}^{\prime} \cdots X_{r}^{\prime} Y_{1} Y_{2} \cdots Y_{s} \bmod _{(r+s-1)}(\mathfrak{G},
$$

where all the products are in $(5$. Hence our assertion is an immediate consequence of the following well-known fact (see [4(a), p. 902]).

LEMMA 3. Let $Z_{1}, \cdots, Z_{d}$ be elements of $g_{c}$ and $\left(i_{1}, i_{2}, \cdots, i_{d}\right)$ a permutation of $(1,2, \cdots, d)$. Then

$$
Z_{1} Z_{2} \cdots Z_{d}-Z_{i} Z_{i_{2}} \cdots Z_{i}, \epsilon_{(d-1)}^{(\mathfrak{G} .}
$$

As usual we regard elements of $\mathfrak{G}$ as left-invariant differential operators on $G$. Moreover, for every $X \in \mathfrak{g}$, let $\rho(X)$ denote the right-invariant vector-field on $G$ given by (3)

$$
f(x ; \rho(X))=(d f(\exp t X \cdot x) / d t)_{t=0} \quad\left(x \in G, f \in C^{\infty}(G)\right) .
$$

A simple argument shows that $[\rho(X), \rho(Y)]=-\rho([X, Y])$ and therefore $\rho$ can be extended uniquely to an anti-homomorphism of $(5$ into the algebra of all differential operators on $G$. We define

$$
f(g ; x)=f(x ; \rho(g))
$$

for $x \in G, g \in \mathbb{G}$ and $f \in C^{\infty}(G)$. If $X_{1}, \cdots, X_{r} \in \mathfrak{g}$, then(4)

$$
\begin{aligned}
f\left(X_{1} X_{2} \cdots X_{r} ; x\right) & =f\left(x ; \rho\left(X_{1} X_{2} \cdots X_{r}\right)\right)=f\left(x ; \rho\left(X_{r}\right) \cdots \rho\left(X_{2}\right) \rho\left(X_{1}\right)\right) \\
& =\left\{\partial^{r} f\left(\exp t_{1} X_{1} \cdots \exp t_{r} X_{r} \cdot x\right) / \partial t_{1} \cdots \partial t_{r}\right\}_{t_{1}=\cdots=t_{r}=0} \\
& =f\left(x ;\left(X_{1} X_{2} \cdots X_{r}\right)^{x^{-1}}\right)
\end{aligned}
$$

since $\exp t_{1} X_{1} \cdots \exp t_{r} X_{r} \cdot x=x\left(\exp t_{1} X_{1} \cdots \exp t_{r} X_{r}\right)^{x^{-1}}$. Therefore

$$
f(g ; x)=f\left(x ; g^{x^{-1}}\right) \quad(g \in(5) .
$$

It is obvious that $X$ and $\rho(Y)(X, Y \in \mathfrak{g})$ commute (in the algebra of differential operators on $G)$ and therefore $g_{1}$ and $\rho\left(g_{2}\right)\left(g_{1}, g_{2} \in(\mathfrak{F})\right.$ also commute.

Now $G$ operates on itself by means of inner automorphisms so that $y^{x}=x y x^{-1}$ $(x, y \in G$, see $[4(\mathrm{k}), \S 5])$. Let $\Omega_{0}$ and $G_{0}$ be two open sets in $G$ and $f$ a $C^{\infty}$-function on $\Omega=\Omega_{0}{ }^{G_{0}}$. Put $f(x: y)=f\left(y^{x}\right)\left(x \in G_{0}, y \in \Omega_{0}\right)$. The significance of the mapping $\Gamma_{y}$ arises from the following lemma.

Lemma 4. Let $g_{1}, g_{2} \in(5$. Then

$$
f\left(x ; g_{1}: y ; g_{2}\right)=f\left(x: y ; \Gamma_{y}\left(g_{1} \otimes g_{2}\right)\right)
$$

for $x \in G_{0}$ and $y \in \Omega_{0}$.

(3) We use here the notation of [4(k), §2].

(4) For any $x \in G$, we extend $\operatorname{Ad}(x)$ to an automorphism $g \rightarrow g^{x}$ of $(5$ and define $y^{x}=x y x^{-1}(y \in G)$. 
If $X \in \mathfrak{g}$, it is clear that

$$
y^{\exp t X}=y \exp \left(t X^{y^{-1}}\right) \exp (-t X) \quad(y \in G, t \in R) .
$$

On the other hand if $x \in G_{0}$ and $y \in \Omega_{0}$, it is clear that

$$
f(x \exp t X: y)=f\left(x: y^{\exp t X}\right)
$$

provided $|t|$ is small. Therefore

$$
f(x ; X: y)=f\left(x: y ; X^{y^{-1}}-X\right)=f(x: y ; \rho(X)-X) .
$$

Since $\rho(X)$ commutes with $g_{2}$, it follows by differentiation with respect to $y$ that

$$
\begin{aligned}
f\left(x ; X: y ; g_{2}\right) & =f\left(x: y ; \rho(X) \circ g_{2}-g_{2} X\right) \\
& =f\left(x: y ; X^{y^{-1}} g_{2}-g_{2} X\right)=f\left(x: y ; \sigma_{y}(X) g_{2}\right) .
\end{aligned}
$$

Hence if $X_{1}, \cdots, X_{r} \in \mathrm{g}$ and $g_{1}=X_{1} X_{2} \cdots X_{r}$, it follows by induction on $r$ that

$$
f\left(x ; g_{1}: y ; g_{2}\right)=f\left(x: y ; \sigma_{y}\left(g_{1}\right) g_{2}\right) .
$$

The statement of the lemma is now obvious.

3. Completely invariant sets. Assume that $G$ is connected. Consider the polynomial

$$
\operatorname{det}(t+1-\operatorname{Ad}(x))=\sum_{0 \leqq j \leqq n} t^{j} D_{j}(x) \quad(x \in G),
$$

where $t$ is an indeterminate and $n=\operatorname{dim} G$. Then $D_{j}$ are analytic functions on $G$ and $D_{n}=1$. Let $l$ be the least integer such that $D_{l} \neq 0$. Then $l=\operatorname{rank} G=\operatorname{rank} g$ and an element $x \in G$ is called singular or regular according as $D_{l}(x)=0$ or not. Let $G^{\prime}$ be the set of all regular elements. Then it is obvious that $G^{\prime}$ is open and dense in $G$ and the set of singular elements is of measure zero with respect to the leftinvariant Haar measure of $G$.

We say that $G$ is reductive if $\mathfrak{g}$ is reductive. An element $x \in G$ is called semisimple if the endomorphism $\operatorname{Ad}(x)$ of $g$ is semisimple. Let $z_{x}$ denote the centralizer of $x$ in $g$. We assume, from now on, that $G$ is reductive.

LEMMA 5. Let $x$ be an element of $G$. Then $x \in G^{\prime}$ if and only if $z_{x}$ is a Cartan subalgebra of $\mathfrak{g}$. Moreover, if $x$ is semisimple then $z_{x}$ is reductive in $\mathfrak{g}$ and rank $z_{x}=$ rank $\mathfrak{g}$. Finally, a regular element is always semisimple.

It is clearly enough to consider the case when $g$ is semisimple. The first and last statements follow from [4(e), Lemma 5].Put $B(X, Y)=\operatorname{tr}(\operatorname{ad} X$ ad $Y)(X, Y \in g)$ and let $B_{x}$ denote the restriction of the bilinear form $B$ on $z_{x}$. Now assume that $X$ is semisimple. An elementary argument (see $\left[2\right.$, p. 391]) shows that $B_{x}$ is non- 
degenerate. Hence it follows from [2, Proposition 3.4] and [3] that $z_{x}$ is reductive in $\mathfrak{g}$. Finally rank $z_{x}=\operatorname{rankg}$ from [2, Proposition 4.6].

Corollary. If $x$ is semisimple, it is contained in a Cartan subgroup of $G$.

Let $\mathfrak{h}$ be a Cartan subalgebra of $z_{x}$ and $A$ the centralizer of $\mathfrak{h}$ in $G$. Since rank $3_{x}=\operatorname{rank} g, A$ is a Cartan subgroup of $G$ and $x \in A$.

Let $\mathscr{N}$ denote the set of all nilpotent elements (see $[4(\mathrm{n}), \S 3]$ ) of $\mathrm{g}$. Put $\mathscr{N}_{G}=\exp \mathscr{N} \subset G$. The mapping $X \rightarrow \operatorname{expad} X(X \in \mathscr{N})$ is known (see $\left.[4(\mathrm{~h}), \S 3]\right)$ to be univalent on $\mathscr{N}$.

LEMMA 6. Every $x \in G$ can be written uniquely in the form $x=h n$, where $h$ is a semisimple element of $G, n \in \mathscr{N}_{G}$ and $h, n$ commute with each other. Let $Z_{x}$ denote the centralizer of $x$ in $G$. Then $h$ and $n$ lie in the center of $Z_{x}$.

It is obviously enough to consider the case when $g$ is semisimple and $G$ is the connected component of 1 in the adjoint group $G_{0}$ of $\mathfrak{g}$. Then $G_{0}$ is the set of all real points of a linear algebraic group defined over $\boldsymbol{R}$. Therefore the lemma follows from well-known results on algebraic groups (see, for example, $[1, \S 8]$ ). $h$ and $n$, respectively, are called the semisimple and unipotent components of $x$.

Corollary(5). $h \in \mathrm{Cl}\left(x^{G}\right)$.

Choose $X \in \mathscr{N}$ such that $n=\exp X$ and let $z$ denote the centralizer of $h$ in $\mathfrak{g}$. Then $X \in 3$. We may obviously assume that $X \neq 0$. Then by the JacobsonMorosow theorem, we can choose $H \in\{$ such that $[H, X]=2 X$ (see $[4(\mathrm{n}), \S 3]$ ). Put $y_{t}=\exp (-t H)$. Then

$$
x^{y_{t}}=(h \exp X)^{y_{t}}=h \exp \left(e^{-2 t} X\right) \rightarrow h
$$

as $t \rightarrow+\infty$. This proves the corollary.

Let $U$ be a subset of $G$. We say that $U$ is completely invariant (cf. [4(n), §3]) if it has the following property. If $C$ is any compact subset of $U$, then $\mathrm{Cl}\left(C^{G}\right) \subset U$.

LEMMA 7. Let $U$ be a completely invariant subset of $G$ and $V$ an invariant subset of $U$ which is closed in $U$. Then if $V$ contains no semisimple element of $U, V$ is empty.

Suppose $x \in V$. Then it follows from the corollary of Lemma 6 that the semisimple component of $x$ also lies in $V$. Hence the lemma.

Now assume that $\mathfrak{g}$ is semisimple. For any $c>0$, let $g(c)$ denote the set of all $X \in \mathfrak{g}$ such that (6) $|\operatorname{Im} \lambda|<c$ for every eigenvalue $\lambda$ of $\operatorname{ad} X$. Clearly $\mathfrak{g}(c)$ is an open and completely invariant neighborhood of zero in $\mathfrak{g}$ and $\mathscr{N} \subset \mathfrak{g}(c)$. Moreover, if $X \in \mathfrak{g}(c)$ then $t X \in \mathfrak{g}(c)$ for $0 \leqq t \leqq 1$. Hence $\mathfrak{g}(c)$ is connected.

(5) As usual $\mathrm{Cl} X$ and ${ }^{\mathrm{c}} X$, respectively, denote the closure and the complement of a subset $X$,

( $\left.{ }^{\circ}\right) \operatorname{Im} \lambda$ denotes, as usual, the imaginary part of $\lambda$. 
Lemma 8. Assume that $c \leqq \pi$. Then the exponential mapping from $g$ into $G$ is everywhere regular and univalent on $\mathrm{g}(c)$. Moreover, exp $\mathrm{g}(c)$ is completely invariant in $G$.

The proof of the first part is the same as that of [4(h), Lemma 11]. In order to obtain the second part we use the notation of [4(h), Lemma 12] and first prove the following lemma.

Lemma 9. Assume $c \leqq \pi$ and let $X_{r}(r \geqq 1)$ be a sequence in $g(c)$. Then if $\left\|\exp X_{r}\right\|$ remains bounded, the same holds for $\left\|X_{r}\right\|$.

We keep to the notation of the proof of [4(h), Lemma 12]. Then $X_{r}=\operatorname{Ad}\left(u_{r}\right) Y_{r}$, $Y_{r}=H_{r}+Z_{r}$ and therefore $\left|\operatorname{Im} \alpha\left(H_{r}\right)\right|<c \leqq \pi$ for any $\alpha \in P$. On the other hand $\left\|\exp X_{r}\right\|=\left\|\exp Y_{r}\right\|$ and ad $Y_{r}$ has the same eigenvalues as $\operatorname{ad} H_{r}$. This shows that $\left|e^{\left.x_{(} H_{r}\right)}\right|$ remains bounded for every $\alpha \in P$. In view of the above result this implies that $\left\|H_{r}\right\|$ itself remains bounded. The rest of the proof now goes through in the same way as for Lemma 12 of [4(h)].

Now fix a compact set $C$ in $V=\operatorname{expg}(c)$. We have to show that $\mathrm{Cl}\left(C^{G}\right) \subset V$. Let $X_{k}$ and $x_{k}(k \geqq 1)$ be sequences in $g(c)$ and $G$ respectively such that $\exp X_{k} \in C$ and $\left(\exp X_{k}\right)^{x_{k}}$ converges to some point $y$ in $G$. We have to verify that $y \in V$. Let $\log$ denote the inverse mapping from $V$ to $g(c)$. Then $\log C$ is compact. Hence, in view of Lemma 9, we can, by choosing suitable subsequences, arrange that $X_{k} \rightarrow X$ and $x_{k} X_{k} \rightarrow Y$, where $X \in \log C$ and $Y \in \mathfrak{g}$. But it is obvious that ad $X$ and $\operatorname{ad} Y$ have the same eigenvalues. Hence $Y \in \mathfrak{g}(c)$ and therefore $y=\exp Y \in V$. This completes the proof of Lemma 8.

4. Some algebraic results $\left({ }^{7}\right)$. We return again to the case when $g$ is reductive. Fix a semisimple element $a \in G$ and let $z=z(a)$ denote the centralizer of $a$ in $g$ and $\Xi=\Xi(a)$ the analytic subgroup of $G$ corresponding to $z$. Put

$$
v_{a}(y)=\operatorname{det}\left(\operatorname{Ad}(a y)^{-1}-1\right)_{\mathfrak{g} / \mathbf{3}} \quad(y \in \Xi) .
$$

Then $v_{a}$ is an analytic function on $\Xi$ and $v_{a}(1) \neq 0$. Let $\Xi^{\prime}=\Xi^{\prime}(a)$ be the set of all points $y \in \Xi$, where $v_{a}(y) \neq 0$. Then $\Xi^{\prime}$ is an open neighborhood of 1 in $\Xi$.

In view of Lemma 5,3 satisfies the conditions of [4(1), §2]. Define $q$ as in [4(1), §2] and put $\mathfrak{Q}=\mathfrak{S}\left(\mathfrak{q}_{c}\right)$ and $\mathfrak{Q}_{+}=\mathfrak{S}_{+}\left(\mathfrak{q}_{c}\right)$ in the notation of [4(k), §7].

Lemma 10. Fix $y \in \Xi^{\prime}$. Then $\Gamma_{a y}$ defines a bijective mapping of $\mathbb{Q} \otimes \mathfrak{S}\left(z_{c}\right)$ onto (5. Moreover,

$$
\sum_{d_{1}+d_{2} \leqq d} \Gamma_{a y}\left(\Im_{d_{1}}\left(\mathfrak{q}_{c}\right) \otimes \mathfrak{S}_{d_{2}}\left(\mathfrak{z}_{c}\right)\right)={ }_{d}(\mathfrak{G} \quad(d \geqq 0) .
$$

Put $W_{d}=\Sigma_{d_{1}+d_{2} \leqq d} \Im_{d_{1}}\left(\mathfrak{q}_{c}\right) \otimes \mathfrak{S}_{d_{2}}\left(\mathfrak{z}_{c}\right)$. Since $\mathfrak{g}$ is the direct sum of $\mathfrak{q}$ and $\mathfrak{z}$, it is clear that $\operatorname{dim} W_{d}=\operatorname{dim}_{d}(\mathfrak{G}$. Hence it would be sufficient to prove that

(7) The results of this section are similar to those of [4(1), §2]. See also [4(e)]. 
$\Gamma_{a y}\left(W_{d}\right)={ }_{d}\left(\mathfrak{F}\right.$. We do this by induction on $d$. It is obvious that $\Gamma_{a y}\left(W_{d}\right) \subset_{d}(\mathfrak{b}$. Hence it would be sufficent to show that

$$
{ }_{d}\left(\mathfrak{5} \subset \Gamma_{a y}\left(W_{d}\right)+{ }_{(d-1)}(\mathfrak{5} .\right.
$$

Fix two integers $d_{1}, d_{2} \geqq 0$ such that $d_{1}+d_{2}=d$ and suppose $Y_{i} \in \mathfrak{q}\left(1 \leqq i \leqq d_{1}\right)$ and $Z_{j} \in z\left(1 \leqq j \leqq d_{2}\right)$. Let $q=Y_{1} Y_{2} \cdots Y_{d_{1}} \in S\left(\mathfrak{q}_{c}\right)$ and $z=Z_{1} Z_{2} \cdots Z_{d_{2}} \in S\left(z_{c}\right)$. (Here we have to take $q=1$ if $d_{1}=0$ and $z=1$ if $d_{2}=0$.) Define $\lambda$ as in $\S 2$. Then it would be enough to verify that

$$
\lambda(q z) \in \Gamma_{a y}\left(W_{d}\right)+{ }_{(d-1)}(\mathfrak{b} .
$$

If $d_{1}=0$, this is obvious since $\Gamma_{a y}(1 \otimes \lambda(z))=\lambda(z)$. Hence we may assume $d_{1}>0$. Now $a y$ commutes with $a$ and therefore $z^{a y}=z$ and $\mathfrak{q}^{a y}=\mathfrak{q}$ (see [4(1), §2]). Therefore since $v_{a}(y) \neq 0$, we can choose $Y_{i}^{\prime} \in \mathfrak{q}$ such that $\left(\operatorname{Ad}(a y)^{-1}-1\right) Y_{i}^{\prime}=Y_{i}$ $\left(1 \leqq i \leqq d_{1}\right)$. Put $q^{\prime}=Y_{1}^{\prime} \cdots Y_{r}^{\prime} \in S\left(\mathfrak{q}_{c}\right)$. Then

$$
\Gamma_{a y}\left(\lambda\left(q^{\prime}\right) \otimes \lambda(z)\right) \equiv \lambda(q z) \bmod (d-1)(\mathfrak{b}
$$

from Lemma 2. This proves the lemma.

Corollary 1. Fix $g \in \mathfrak{G}$. Then for any $y \in \Xi^{\prime}$, there exist unique elements $\alpha_{y}(g) \in \mathfrak{S}\left(z_{c}\right)$ and $\beta_{y}(g) \in \mathfrak{Q}_{+} \otimes \mathfrak{S}\left(z_{c}\right)$ such that

$$
g=\alpha_{y}(g)+\Gamma_{a y}\left(\beta_{y}(g)\right) \text {. }
$$

Moreover, if $g \in{ }_{d}\left(\mathfrak{F}\right.$, then $d^{0} \alpha_{y}(g) \leqq d$ and

$$
\beta_{y}(g) \in \sum_{d_{2} \geqq 0} \sum_{1 \leqq d_{1} \leqq d-d_{2}} \Im_{d_{1}}\left(\mathfrak{q}_{c}\right) \otimes \mathfrak{S}_{d_{2}}\left(z_{c}\right) .
$$

This is obvious from Lemma 10.

Let $M$ be an analytic manifold and $f$ a mapping of $M$ into a complex vector space $V$. We say that $f$ is analytic if the subspace $U$ of $V$ spanned by the image $f(M)$ is of finite dimension and $f$, viewed as a mapping of $M$ into $U$, is analytic in the usual sense.

COROLlaRY 2. Given $g \in(5$, we can choose an integer $r \geqq 0$ such that the mappings $y \rightarrow v_{a}(y)^{r} \alpha_{y}(g)$ and $y \rightarrow v_{a}(y)^{r} \beta_{y}(g)\left(y \in \Xi^{\prime}\right)$ can be extended to analytic mappings of $\Xi$ into $\mathfrak{S}\left(\mathfrak{z}_{c}\right)$ and $\mathfrak{Q}_{+} \otimes \mathfrak{S}\left(\mathfrak{z}_{c}\right)$, respectively.

Let $d=d^{0} g$. If $d=0$ our statement is obvious. So we assume that $d \geqq 1$ and use induction. We may obviously assume that $g=\lambda(q z)$ in the notation of the proof of Lemma 10. Let $A(y)$ denote the restriction of $\left(\operatorname{Ad}(a y)^{-1}-1\right)$ on $q(y \in \Xi)$. Then if $t$ is an indeterminate,

$$
\operatorname{det}(t-A(y))=\sum_{0 \leqq k \leqq m} D_{k}(y) t^{k}
$$

Here $m=\operatorname{dim} q, D_{k}(0 \leqq k \leqq m)$ are analytic functions on $\Xi, D_{m}=1$ and 
Therefore

$$
D_{0}(y)=(-1)^{m} \operatorname{det} A(y)=(-1)^{m} v_{a}(y) .
$$

where

$$
v_{a}(y)=B(y) A(y)=A(y) B(y),
$$

$$
B(y)=(-1)^{m+1} \sum_{0 \leqq k<m} D_{k+1}(y) A(y)^{k} .
$$

Put $Y_{i}(y)=B(y) Y_{i}\left(1 \leqq i \leqq d_{1}\right)$ and $q(y)=\prod_{1 \leqq i \leqq d_{1}} Y_{i}(y) \in S\left(\mathfrak{q}_{c}\right)(y \in \Xi)$. Then it follows from Lemma 2 that

$$
v(y)=\Gamma_{a y}(\lambda(q(y)) \otimes z)-v_{a}(y)^{d_{1}} \lambda(q z) \epsilon_{(d-1)}(\mathfrak{G} .
$$

Therefore

$$
v(y)=\sum_{1 \leqq i \leqq r} a_{i}(y) v_{i}
$$

where $a_{i}$ are analytic functions on $\Xi$ and $d^{0} v_{i}<d$. The required result now follows immediately by applying the induction hypothesis to $v_{i}(1 \leqq i \leqq r)$.

COROLlaRY 3. Let $Z_{a}$ denote the centralizer of $a$ in $G$. Then if $x \in Z_{a}, y \in \Xi^{\prime}$ and $g \in \mathfrak{G}$, we have

$$
\alpha_{x y x-1}\left(g^{x}\right)=\left(\alpha_{y}(g)\right)^{x} .
$$

This follows immediately from Lemma 1 .

5. The mapping $\delta_{a, G / \Xi}$. We keep to the notation of $\S 4$. Let $U_{G}$ be an open neighborhood of $a$ in $G$. Put $U_{\Xi}=\Xi^{\prime} \cap\left(a^{-1} U_{G}\right)$. Then $U_{\Xi}$ is an open neighborhood of 1 in $\Xi$. For any differential operator $D$ on $U_{G}$, we define a differential operator $\Delta(D)$ on $U_{\Xi}$ as follows:

$$
(\Delta(D))_{y}=\alpha_{y}\left(D_{a y}\right) \quad\left(y \in U_{\Xi}\right) .
$$

Here $D_{a y}$ and $(\Delta(D))_{y}$ denote, as usual, the local expressions (see [4(e), p. 112]) of $D$ at $a y$ and $\Delta(D)$ at $y$, respectively. Corollary 2 of Lemma 10 insures that there does exist a differential operator $\Delta(D)$ on $U_{\Xi}$ satisfying the above relation and it is analytic if $D$ is analytic. Finally, if we assume that $U_{G}$ and $D$ are invariant under $G$ (see [4(j), §2]), it follows from Corollary 3 of Lemma 10 that $U_{\Xi}$ and $\Delta(D)$ are invariant under $Z_{a}$. We shall denote the mapping $D \rightarrow \Delta(D)$ by $\delta_{a}$ or, if necessary, by $\delta_{a, G / \Xi}$.

Let $b$ be an element of $U_{\Xi}$ which is regular in $\Xi$ and let $\mathfrak{h}$ denote the centralizer of $b$ in $\mathfrak{z}$. Then $\mathfrak{h}$ is a Cartan subalgebra of $\mathfrak{z}$ and therefore also of $\mathfrak{g}$ (see Lemma 5). Let $A_{\mathfrak{h}}$ denote the Cartan subgroup of $G$ corresponding to $\mathfrak{h}$ (see [4(m), 85$]$ ). Then $a, b$ are in $A_{\mathfrak{h}}$. Let $A$ be the connected component of 1 in $A_{\mathfrak{h}}$.

LeMma 11. The following two conditions on an element $c$ of $A$ are mutually equivalent.

(1) $c \in b^{-1} U_{3}$ and $\operatorname{det}\left(\operatorname{Ad}(b c)^{-1}-1\right)_{3 / \mathfrak{b}} \neq 0$.

(2) $c \in(a b)^{-} U_{G}$ and $\operatorname{det}\left(\operatorname{Ad}(a b c)^{-1}-1\right)_{\mathbf{g} / \mathfrak{b}} \neq 0$. 
Since $\operatorname{Ad}(a)=1$ on 3 , it is clear that

$$
\operatorname{det}\left(\operatorname{Ad}(a b c)^{-1}-1\right)_{\mathfrak{g} / \mathfrak{h}}=\operatorname{det}\left(\operatorname{Ad}(a b c)^{-1}-1\right)_{\mathfrak{g} / \mathfrak{z}} \operatorname{det}\left(\operatorname{Ad}(b c)^{-1}-1\right)_{3 / \mathfrak{h}}
$$

for $c \in A$. Now suppose (1) holds. Then $b c \in U_{\Xi}$ and therefore $a b c \in U_{G}$ and $\operatorname{det}\left(\operatorname{Ad}(a b c)^{-1}-1\right)_{\mathfrak{g} / 3} \neq 0$. Therefore (1) implies (2). Conversely, assume that (2) holds. Then $b c \in a^{-1} U_{G}$ and

$$
v_{a}(b c) \operatorname{det}\left(\operatorname{Ad}(b c)^{-1}-1\right)_{3 / \mathfrak{b}} \neq 0 .
$$

Hence $b c \in U_{\Xi}$ and (1) holds.

COROLlaRY. $a b$ is regular in $G$ and $\mathfrak{h}$ is the centralizer of $a b$ in $\mathfrak{g}$.

Take $c=1$ in Lemma 11. Then condition (1) obviously holds and therefore $\operatorname{det}\left(\operatorname{Ad}(a b)^{-1}-1\right)_{\mathfrak{g} / \mathfrak{h}} \neq 0$ by (2). Since $\operatorname{Ad}(a b)=1$ on $\mathfrak{h}$, it follows that $\mathfrak{h}$ is the centralizer of $a b$ in $\mathrm{g}$. Therefore $a b$ is regular in $G$ by Lemma 5 .

Let $U_{A}$ be the set of all $c \in A$ satisfying the conditions of Lemma 11 .

LEMMA 12. Let $D$ be a differential operator on $U_{G}$. Then( $\left(^{8}\right)$

$$
\delta_{a b, G / A}(D)=\delta_{b, \Xi / A}\left(\delta_{a, G / \Xi}(D)\right) .
$$

It follows from Lemma 11 that both sides are differential operators on $U_{A}$. Let $\Delta_{1}=\delta_{a, G / \Xi}(D), \Delta_{2}=\delta_{b, \Xi / A}\left(\Delta_{1}\right)$ and $\Delta=\delta_{a b, G / A}(D)$. We have to prove that $\Delta_{2}=\Delta$. Let $\mathfrak{m}=[\mathfrak{h}, \mathfrak{z}]$ and $\mathfrak{p}=\mathfrak{q}+\mathfrak{m}$. Then $\mathfrak{g}=\mathfrak{h}+\mathfrak{p}$ and $\mathfrak{z}=\mathfrak{h}+\mathfrak{m}$ where both sums are direct.

Fix $h \in U_{A}$. Then

$$
\left(\Delta_{2}\right)_{h}-\left(\Delta_{1}\right)_{b h} \in \Gamma_{b h}\left(\Im_{+}\left(m_{c}\right) \otimes \Im\left(\mathfrak{h}_{c}\right)\right) .
$$

On the other hand $b h \in U_{\Xi}$ and therefore

$$
\left(\Delta_{1}\right)_{b h}-D_{a b h} \in \Gamma_{a b h}\left(\Im_{+}\left(\mathfrak{q}_{c}\right) \otimes \Im\left(z_{c}\right)\right) .
$$

Since $\operatorname{Ad}(a)=1$ on 3 , it is clear that

and therefore

$$
\sigma_{a b h}(z)=\sigma_{b h}(z) \quad\left(z \in \Im\left(z_{c}\right)\right)
$$

$$
\left(\Delta_{2}\right)_{h}-D_{a b h} \in \Gamma_{a b h}\left(\mathfrak{G}_{+} \otimes \mathfrak{S}\left(\mathfrak{z}_{c}\right)\right),
$$

where $\mathfrak{G}_{+}=\mathfrak{S}_{+}\left(\mathfrak{g}_{c}\right)$. But

$$
\Gamma_{a b h}\left(\mathfrak{S}\left(\mathfrak{m}_{c}\right) \otimes \mathfrak{S}\left(\mathfrak{h}_{c}\right)\right)=\Gamma_{b h}\left(\mathfrak{S}\left(\mathfrak{m}_{c}\right) \otimes \mathfrak{S}\left(\mathfrak{h}_{c}\right)\right)=\mathfrak{S}\left(\mathfrak{z}_{c}\right)
$$

from Lemma 10 (applied to $(\Xi, b)$ instead of $(G, a))$, since $\operatorname{det}\left(\operatorname{Ad}(b h)^{-1}-1\right)_{3 / \mathfrak{h}} \neq 0$. Hence

$$
\begin{aligned}
\Gamma_{a b h}\left(\mathfrak{G}_{+} \otimes \mathfrak{S}\left(\mathfrak{z}_{c}\right)\right) & =\sigma_{a b h}\left(\mathfrak{G}_{+}\right) \mathfrak{S}\left(\mathfrak{z}_{c}\right) \\
& =\sigma_{a b h}\left(\mathfrak{G}_{+}\right) \sigma_{a b h}\left(\mathfrak{S}\left(\mathfrak{m}_{c}\right)\right) \Im\left(\mathfrak{h}_{c}\right) \\
& =\sigma_{a b h}\left(\mathfrak{G}_{+}\right) \Im\left(\mathfrak{h}_{c}\right) .
\end{aligned}
$$

(8) Cf. [4(1), Lemma 11]. 
But $\mathfrak{b}=\mathfrak{S}\left(\mathfrak{p}_{c}\right) \mathfrak{S}\left(\mathfrak{h}_{c}\right)$ and since $\mathfrak{h}$ is abelian, it is clear that

Therefore since

$$
\sigma_{a b h}\left(\mathfrak{S}_{+}\left(\mathfrak{h}_{c}\right)\right) \Im\left(\mathfrak{h}_{c}\right)=\{0\} .
$$

we conclude that

$$
\mathfrak{G}_{+}=\mathfrak{S}_{+}\left(\mathfrak{p}_{c}\right)+\mathfrak{S}\left(\mathfrak{p}_{c}\right) \mathfrak{S}_{+}\left(\mathfrak{h}_{c}\right)
$$

This shows that

$$
\Gamma_{a b h}\left(\mathfrak{G}_{+} \otimes \mathfrak{S}\left(\mathfrak{z}_{c}\right)\right)=\Gamma_{a b h}\left(\mathfrak{S}_{+}\left(\mathfrak{p}_{c}\right) \otimes \mathfrak{S}\left(\mathfrak{h}_{c}\right)\right)
$$

$$
\left(\Delta_{2}\right)_{h}-D_{a b h} \in \Gamma_{a b h}\left(\mathfrak{S}_{+}\left(\mathfrak{p}_{c}\right) \otimes \mathfrak{S}\left(\mathfrak{h}_{c}\right)\right)
$$

and therefore $\left(\Delta_{2}\right)_{h}=\Delta_{h}$ from the definition of $\Delta$.

6. The case when $a$ is regular. Let 3 be the algebra of all differential operators on $G$ which are invariant under both left and right translations of $G$. It is obvious that 3 consists of the center of $\mathbb{6}$ and therefore 3 is abelian.

Let $G^{\prime}$ be the set of all regular elements of $G$. Fix $a \in G^{\prime}$ and let $\mathfrak{h}$ denote the centralizer of $a$ in $\mathfrak{g}$ and $A$ the analytic subgroup of $G$ corresponding to $\mathfrak{h}$. Then $\mathfrak{h}$ is a Cartan subalgebra of $\mathfrak{g}$ and

$$
v_{a}(h)=\operatorname{det}\left(\operatorname{Ad}(a h)^{-1}-1\right)_{\mathfrak{g} / \mathfrak{h}} \quad(h \in A) .
$$

Hence $A^{\prime}=A \cap\left(a^{-1} G^{\prime}\right)$ is the set of all points $h \in A$ where $v_{a}(h) \neq 0$. Let $W$ be the Weyl group of $\left(\mathfrak{g}_{c}, \mathfrak{h}_{c}\right)$. Then $W$ operates on $\mathfrak{S}\left(\mathfrak{h}_{c}\right)$. Let $I\left(\mathfrak{h}_{c}\right)$ be the algebra of all invariants of $W$ in $\mathfrak{S}\left(\mathfrak{h}_{c}\right)$. We have a canonical isomorphism $\gamma$ of 3 onto $I\left(\mathfrak{h}_{c}\right)$ (see [4(e), Lemma 19]). Thus for every $z \in \mathcal{Z}, \gamma(z)$ is a differential operator on $A$ which is invariant under the translations of $A$.

LEMMA 13. $\delta_{a, G / A}(z)=\left|v_{a}\right|^{-1 / 2} \gamma(z) \circ\left|v_{a}\right|^{1 / 2}$ on $A^{\prime}$ for any $z \in 3$.

This is substantially the same as the first statement of [4(e), Theorem 2, p. 125].

7. Application to invariant distributions( $\left({ }^{9}\right)$. Fix a semisimple element $a \in G$ and define $\Xi$ and $\Xi^{\prime}$ as in $\$ 4$.

LEMMA 14. Consider the mapping $\phi:(x, y) \rightarrow(a y)^{x}$ of $G \times \Xi$ into $G$. Then if $n=\operatorname{dim} G, \phi$ is everywhere of rank $n$ on $G \times \Xi^{\prime}$.

We identify the tangent space of $G \times \Xi$ at a point $(x, y)$ with $g \times z$ in the usual way. Then a simple calculation shows that

$$
(d \phi)_{x, y}(X, Z)=\left(Z+\left(\operatorname{Ad}(a y)^{-1}-1\right) X\right)^{x}
$$

for $X \in \mathfrak{g}$ and $Z \in \mathfrak{z}$. But

$$
\mathfrak{z}+\left(\operatorname{Ad}(a y)^{-1}-1\right) \mathfrak{q}=\mathfrak{z}+\mathfrak{q}=\mathfrak{g}
$$

if $y \in \Xi^{\prime}$ and therefore our assertion is obvious.

(9) The results of this section are similar to those of [4(1), §7]. 
Let $d x$ denote the Haar measure on $G$. We orient $G$ and fix a left-invariant differential form $\omega_{G}>0$ of degree $n$ on $G$, corresponding to the measure $d x$. Then the set up of $[4(\mathrm{k}), \S 5]$ is applicable to $M=G$, if we define $y^{x}=x y x^{-1}(x, y \in G)$ as above.

Let $U$ be an open neighborhood of 1 in $\Xi^{\prime}$ which is invariant under $\Xi$ (i.e., $U^{y}=U$ for $\left.y \in \Xi\right)$. Put $\Omega=\phi(G \times U)=(a U)^{G}$. Then by Lemma $14, \Omega$ is open in $G$. Let $d x, d y$ denote the Haar measures on $G$ and $\Xi$, respectively. Now take $M=G \times U, N=\Omega, \pi=\phi$ in Theorem 1 of $[4(\mathrm{k})]$ and let $\omega_{M}$ and $\omega_{N}$ be the differential forms corresponding to the measures $d x d y$ and $d x$, respectively. Let $\alpha \rightarrow f_{\alpha}$ denote the corresponding mapping of $C_{c}^{\infty}(G \times U)$ onto $C_{c}^{\infty}(\Omega)$.

LEMMA 15. Let $T$ be an invariant distribution on $\Omega$. Then there exists a unique distribution $\sigma_{T}$ on $U$ such that $T\left(f_{\alpha}\right)=\sigma_{T}\left(\beta_{\alpha}\right)\left(\alpha \in C_{c}{ }^{\infty}(G \times U)\right)$, where

$$
\beta_{\alpha}(y)=\int \alpha(x: y) d x \quad(y \in U) .
$$

Moreover, $\sigma_{T}$ is invariant under $\Xi$ and $\sigma_{T}=0$ implies that $T=0$.

Define $T^{\prime}(\alpha)=T\left(f_{\alpha}\right)\left(\alpha \in C_{c}{ }^{\infty}(G \times U)\right)$. Then (see [4(k), Lemma 5]) $T^{\prime}$ is a distribution on $G \times U$. Fix $x_{0} \in G$ and let $\alpha_{x_{0}}$ denote the function $(x, y) \rightarrow \alpha\left(x_{0} x: y\right)$ on $G \times U$. We claim that $T^{\prime}(\alpha)=T^{\prime}\left(\alpha_{x_{0}}\right)$. For if $F \in C_{c}{ }^{\infty}(\Omega)$, we have

$$
\begin{aligned}
\int f_{\alpha_{x_{0}}} F d x & =\int \alpha_{x_{0}}(x: y) F\left((a y)^{x}\right) d x d y=\int \alpha(x: y) F^{x_{0}}\left((a y)^{x}\right) d x d y \\
& =\int f_{\alpha} F^{x_{0}} d x=\int f_{\alpha}^{x_{0}-1} F d x .
\end{aligned}
$$

Hence $f_{\alpha_{x_{0}}}=f_{\alpha}^{x_{0}-1}$ and therefore $T^{\prime}\left(\alpha_{x_{0}}\right)=T\left(f_{\alpha_{x_{0}}}\right)=T\left(f_{\alpha}\right)=T^{\prime}(\alpha)$. Now fix $\beta \in C_{c}{ }^{\infty}(U)$ and put $T_{\beta}{ }^{\prime}(\gamma)=T^{\prime}(\gamma \times \beta)\left(\gamma \in C_{c}{ }^{\infty}(G)\right)$. Then $T_{\beta}{ }^{\prime}$ is a distribution on $G$ which is invariant under the left translations of $G$. Hence $T_{\beta}{ }^{\prime}=c(\beta)$, where $c(\beta)$ is a constant (see [4(k), Lemmas 6 and 7]). Now select $\gamma_{0} \in C_{c}{ }^{\infty}(G)$ such that $\int \gamma_{0} d x=1$. Then

$$
c(\beta)=T_{\beta}{ }^{\prime}\left(\gamma_{0}\right)=T^{\prime}\left(\gamma_{0} \times \beta\right) \quad\left(\beta \in C_{c}{ }^{\infty}(U)\right) .
$$

This shows that the mapping $\beta \rightarrow c(\beta)$ is a distribution on $U$ which we denote by $\sigma_{T}$. Then

$$
T^{\prime}(\gamma \times \beta)=\sigma_{T}(\beta) \int \gamma d x \quad\left(\gamma \in C_{c}{ }^{\infty}(G), \beta \in C_{c}{ }^{\infty}(U)\right)
$$

and therefore we conclude from [4(k), Lemma 3] that $T^{\prime}(\alpha)-\sigma_{T}\left(\beta_{\alpha}\right)=0$ for $\alpha \in C_{c}{ }^{\infty}(G)$. Since $\beta_{\alpha}=\beta$ for $\alpha=\gamma_{0} \times \beta$, the mapping $\alpha \rightarrow \beta_{\alpha}$ of $C_{c}{ }^{\infty}(G \times U)$ into $C_{c}{ }^{\infty}(U)$ is surjective. Finally the mapping $\alpha \rightarrow f_{\alpha}$ of $C_{c}{ }^{\infty}(G \times U)$ into $C_{c}{ }^{\infty}(\Omega)$ is also surjective (see [4(k), Theorem 1]) and so all the statements of the lemma, except the invariance of $\sigma_{T}$ under $\Xi$, are now obvious. 
Fix $\xi \in \Xi$ and define $\alpha^{\xi}(x: y)=\alpha\left(x: y^{\xi-1}\right)$. Then we claim that $T^{\prime}(\alpha)=T^{\prime}\left(\alpha^{\xi}\right)$ for $\alpha \in C_{c}{ }^{\infty}(G \times U)$. This is seen as follows.

$$
\begin{aligned}
\int f_{\alpha^{\xi}} F d x & =\int \alpha\left(x: y^{\xi^{-1}}\right) F\left((a y)^{x}\right) d x d y=\int \alpha(x: y) F\left((a y)^{x \xi}\right) d x d y \\
& =\int \alpha\left(x \xi^{-1}: y\right) F\left((a y)^{x}\right) d x d y
\end{aligned}
$$

for any $F \in C_{c}{ }^{\infty}(\Omega)$. Hence if $\alpha^{\prime}(x: y)=\alpha\left(x \xi^{-1}: y\right)$, it is clear that $f_{\alpha^{\xi}}=f_{\alpha^{\prime}}$. Therefore

$$
T^{\prime}\left(\alpha^{5}\right)=T^{\prime}\left(\alpha^{\prime}\right)=\sigma_{T}\left(\beta_{\alpha^{\prime}}\right)
$$

But

$$
\beta_{\alpha^{\prime}}(y)=\int \alpha\left(x \xi^{-1}: y\right) d x=\beta_{\alpha}(y) \quad(y \in U)
$$

by the right-invariance of $d x$. Hence $T^{\prime}\left(\alpha^{5}\right)=\sigma_{T}\left(\beta_{\alpha}\right)=T^{\prime}(\alpha)$. On the other hand

$$
\beta_{\alpha^{\xi}}(y)=\int \alpha\left(x: y^{\xi^{-1}}\right) d x=\beta_{\alpha}\left(y^{\xi^{-1}}\right) \quad(y \in U) .
$$

Therefore $\beta_{\alpha^{\xi}}=\left(\beta_{\alpha}\right)^{\xi}$. Now for a given $\beta \in C_{c}{ }^{\infty}(U)$, we can choose $\alpha \in C_{c}{ }^{\infty}(G \times U)$ such that $\beta=\beta_{\alpha}$. Then

$$
\sigma_{T}(\beta)=T^{\prime}(\alpha)=T^{\prime}\left(\alpha^{\xi}\right)=\sigma_{T}\left(\beta_{\alpha^{\xi}}\right)=\sigma_{T}\left(\beta^{\xi}\right) .
$$

This shows that $\sigma_{T}$ is invariant under $\Xi$.

COROLlARY. Let $D$ be an invariant differential operator on $\Omega$. Then $\sigma_{D T}=\Delta \sigma_{T}$, where $\Delta=\delta_{a}(D)$.

It follows from Corollary 2 of Lemma 10 and the definition of $\Delta$, that we can select $q_{i} \in \mathfrak{S}_{+}\left(\mathfrak{q}_{c}\right), v_{i} \in \mathfrak{S}\left(\mathfrak{z}_{c}\right)$ and $a_{i} \in C^{\infty}(U)(1 \leqq i \leqq r)$ such that

$$
D_{a y}=\Delta_{y}+\sum_{1 \leqq i \leqq r} a_{i}(y) \Gamma_{a y}\left(q_{i} \otimes v_{i}\right) \quad(y \in U) .
$$

Moreover, $\sigma_{D T}\left(\beta_{\alpha}\right)=T\left(D^{*} f_{\alpha}\right)$ for $\alpha \in C_{c}{ }^{\infty}(G \times U)$, where the star denotes the adjoint as usual. Fix $F \in C_{c}{ }^{\infty}(\Omega)$. Then

$$
\int D^{*} f_{\alpha} \cdot F d x=\int f_{\alpha} D F d x=\int \alpha(x: y) F\left((a y)^{x} ; D\right) d x d y .
$$

Put $F(x: u)=F\left(u^{x}\right)$ for any pair $(x, u) \in G \times G$ such that $u^{x} \in \Omega$. Then it is clear from Lemma 4 that

$$
\begin{aligned}
F\left((a y)^{x} ; D\right) & =F\left((a y)^{x} ; D^{x}\right)=F(x: a y ; D) \\
& =F\left(x: a y ; \Delta_{y}\right)+\sum_{1 \leqq i \leqq r} a_{i}(y) F\left(x ; q_{i}: a y ; v_{i}\right)
\end{aligned}
$$

for $x \in G$ and $y \in U$. Put 


$$
\begin{aligned}
& \alpha_{0}(x: y)=\alpha\left(x: y ; \Delta^{*}\right) \\
& \alpha_{i}(x: y)=\alpha\left(x ; q_{i}^{*}: y ;\left(a_{i} v_{i}\right)^{*}\right) \quad(1 \leqq i \leqq r) .
\end{aligned}
$$

Then

$$
\begin{aligned}
\int D^{*} f_{\alpha} \cdot F d x & =\sum_{0 \leqq i \leqq r} \int \alpha_{i}(x: y) F\left((a y)^{x}\right) d x d y \\
& =\sum_{0 \leqq i \leqq r} \int f_{\alpha_{i}} F d x .
\end{aligned}
$$

This proves that

$$
D^{*} f_{\alpha}=\sum_{0 \leqq i \leqq r} f_{\alpha_{i}}
$$

and therefore

$$
T\left(D^{*} f_{\alpha}\right)=\sum_{0 \leqq i \leqq r} \sigma_{T}\left(\beta_{\alpha_{i}}\right) .
$$

Now $\beta_{\alpha_{0}}=\Delta^{*} \beta_{\alpha}$ and if $j$ denotes the distribution on $G$ corresponding to the constant function 1 , it is obvious that $q_{i} j=0$ since $q_{i} \in \mathfrak{G}_{+}$. Hence it follows that $\beta_{\alpha_{i}}=0(1 \leqq i \leqq r)$ and therefore

$$
T\left(D^{*} f_{\alpha}\right)=\sigma_{T}\left(\Delta^{*} \beta_{\alpha}\right) .
$$

This proves that $\sigma_{D T}=\Delta \sigma_{T}$.

For any $X \in \mathfrak{g}$, let $\tau_{G}(X)$ denote the vector-field on $G$ defined by

$$
\tau_{G}(X) f=\left(d f^{\exp t X} / d t\right)_{t=0} \quad\left(f \in C^{\infty}(G)\right) .
$$

Let $V$ be an open subset of $G$. Then the local invariance of a differential operator, a distribution or a $C^{\infty}$-function on $V$ is defined as in [4(k), §5]. Since $\left[\tau_{G}(X), \tau_{G}(Y)\right]=\tau_{G}([X, Y])(X, Y \in g), \tau_{G}$ can be extended (uniquely) to a homomorphism of $(\mathfrak{F}$ into the algebra of all differential operators on $G$.

Let $G_{0}$ and $U_{0}$ be open neighborhoods of 1 in $G$ and $\Xi^{\prime}$, respectively, and put $\Omega_{0}=\left(a U_{0}\right)^{G_{0}}$. Define the mapping $\alpha \rightarrow f_{\alpha}$ of $C_{c}{ }^{\infty}\left(G_{0} \times U_{0}\right)$ onto $C_{c}{ }^{\infty}\left(\Omega_{0}\right)$ as above. Then the following result is proved in the same way as [4(1), Lemma 17] and [4(k), Theorem 3].

LEMMA 16. Assume that $G_{0}$ is connected and $T$ is a locally invariant distribution on $\Omega_{0}$. Then there exists a unique distribution $\sigma_{T}$ on $U_{0}$ such that $T\left(f_{\alpha}\right)=\sigma_{T}\left(\beta_{\alpha}\right)\left(\alpha \in C_{c}{ }^{\infty}\left(G_{0} \times U_{0}\right)\right)$, where

$$
\beta_{\alpha}(y)=\int \alpha(x: y) d x \quad\left(y \in U_{0}\right) .
$$

Moreover, $\sigma_{T}$ is locally invariant (with respect to $\Xi$ ) and $\sigma_{T}=0$ implies that 
$T_{:}=0$. Finally, $\sigma_{D T}=\delta_{a}(D) \sigma_{T}$ for any locally invariant differential operator $D$ on $\Omega_{0}$.

8. Some preparation for Theorem 1 . Let $G_{0}, \Omega_{0}$ and $\Omega$ be three open subsets of $G$ such that $\Omega_{0}{ }^{G_{0}} \subset \Omega$. For any $f \in C^{\infty}(\Omega)$, we write $f(x: y)=f\left(y^{x}\right)\left(x \in G_{0}, y \in \Omega_{0}\right)$ as in $\S 2$.

Lemma 17. Let $f \in C^{\infty}(\Omega)$. Then

$$
f(x ; g: y)=f\left(x: y ; \tau_{G}\left(g^{*}\right)\right)
$$

for $x \in G_{0}, y \in \Omega_{0}$ and $g \in \mathfrak{G}$.

The proof is the same as that of [4(k), Lemma 11].

LEMMA 18. Let $D$ be a differential operator and $f$ a locally invariant $C^{\infty}$ function on an open subset $\Omega$ of $G$. Fix a semisimple element $a \in \Omega$ and define $\Omega_{\Xi}=a^{-1} \Omega \cap \Xi^{\prime}$ in the notation of $\S 4$. Then

$$
f(a y ; D)=f\left(a y ; \delta_{a}(D)\right) \quad\left(y \in \Omega_{\Xi}\right) .
$$

Fix $y_{0} \in \Omega_{\mathrm{g}}$ and choose open neighborhoods $G_{0}$ and $\Omega_{0}$ of 1 and $a y_{0}$, respectively, in $G$ such that $\Omega_{0}^{G_{0}} \subset \Omega$. Put $\Delta=\delta_{a}(D)$. Then it follows from the definition of $\Delta$ that

$$
D_{a y_{0}}-\Delta_{y_{0}}=\sum_{1 \leqq i \leqq r} \Gamma_{a y_{0}}\left(g_{i} \otimes v_{i}\right)
$$

where $g_{i} \in \mathfrak{G}_{+}$and $v_{i} \in \mathfrak{S}\left(3_{c}\right)$. Therefore we conclude from Lemma 4 that

$$
f\left(a y_{0} ; D_{a y_{0}}-\Delta_{y_{0}}\right)=\sum_{1 \leqq i \leqq r} f\left(1 ; g_{i}: a y_{0} ; v_{i}\right) \text {. }
$$

But

$$
f\left(1 ; g_{i}: x\right)=f\left(x ; \tau_{G}\left(g_{i}^{*}\right)\right)=0 \quad\left(x \in \Omega_{0}\right)
$$

from Lemma 17 since $f$ is locally invariant and $g_{i}^{*} \in \mathfrak{G}_{+}$. Therefore $f\left(1 ; g_{i}: x ; v_{i}\right)=0$ for $x \in \Omega_{0}$ and hence

$$
f\left(a y_{0} ; D_{a y_{0}}-\Delta_{y_{0}}\right)=0 .
$$

This proves the lemma.

LEMMA 19. Let $D$ and $\Omega$ be as above. Then the following two conditions on $D$ are equivalent.

(1) $\delta_{a}(D)=0$ for every regular element $a$ in $\Omega$.

(2) For any open subset $\Omega_{0}$ of $\Omega$ and a locally invariant $C^{\infty}$-function $f$ on $\mathbf{\Omega}_{0}, D f=0$.

Suppose (1) holds and $\Omega_{0}$ and $f$ are given as in condition (2). Fix a regular element $a \in \Omega_{0}$. Then it follows from Lemma 18 that

$$
f(a ; D)=f\left(a ; \delta_{a}(D)\right)=0 .
$$


Therefore $D f=0$ on $\Omega_{0}^{\prime}=\Omega_{0} \cap G^{\prime}$. Since $\Omega_{0}{ }^{\prime}$ is obviously dense in $\Omega_{0}$, we conclude that $D f=0$.

Conversely, assume that (2) holds and fix $a \in \Omega \cap G^{\prime}$. Let $\mathfrak{h}$ be the centralizer of $a$ in $g$ and $A$ the analytic subgroup of $G$ corresponding to $\mathfrak{h}$. Put $\Omega_{A}=a^{-1} \Omega \cap A^{\prime}$ where $A^{\prime}$ is the set of all $h \in A$ where

$$
v_{a}(h)=\operatorname{det}\left(\operatorname{Ad}(a h)^{-1}-1\right)_{\mathfrak{g} / \mathfrak{h}} \neq 0 .
$$

Then $\delta_{a}(D)$ is a differential operator on $\Omega_{A}$. Let $x \rightarrow x^{*}$ denote the natural projection of $G$ on $G^{*}=G / A$. Since $A$ is abelian, $(a h)^{x}(x \in G, h \in A)$ depends only on $x^{*}$ and so we may denote it by $(a h)^{x^{*}}$. It follows from Lemma 14 that the mapping $\psi:\left(x^{*}, h\right) \rightarrow(a h)^{x^{*}}$ of $G^{*} \times \Omega_{A}$ into $G$ is everywhere regular. Fix a point $h_{0} \in \Omega_{A}$. Then we can choose open neighborhoods $G_{0}{ }^{*}$ and $U$ of $1^{*}$ and $h_{0}$ in $G^{*}$ and $\Omega_{A}$, respectively, such that $\Omega_{0}=\psi\left(G_{0}{ }^{*} \times U\right) \subset \Omega$ and $\psi$ defines an analytic diffeomorphism of $G_{0}^{*} \times U$ onto the open neighborhood $\Omega$ of $a h_{0}$ in $\Omega$. Fix $\beta \in C_{\infty}(U)$ and define $f \in C^{\infty}\left(\Omega_{0}\right)$ by $f\left(\psi\left(x^{*}, h\right)\right)=\beta(h)\left(x^{*} \in G_{0}{ }^{*}, h \in U\right)$. Then it is obvious that $f$ is locally invariant and therefore $D f=0$ by (2). On the other hand we know from Lemma 18 that

$$
f\left(a h_{0} ; u\right)=f\left(a h_{0} ; D\right)=0,
$$

where $u$ is the local expression of $\delta_{a}(D)$ at $h_{0}$. Since $u \in \mathfrak{S}\left(\mathfrak{h}_{c}\right)$ and $f(a h)=\beta(h)$ $(h \in U)$, it is obvious that $\beta\left(h_{0} ; u\right)=0$. This being true for every $\beta \in C^{\infty}(U)$, we conclude that $u=0$. Since $h_{0}$ was an arbitrary point of $\Omega_{A}$, this proves that $\delta_{a}(D)=0$. Therefore (2) implies (1).

9. First part of the proof of Theorem 1. We shall now begin the proof of the following theorem (cf. [4(n), Theorem 5]).

THEOREM 1. Let $\Omega$ be a completely invariant open set in $G$ and $D$ an analytic differential operator on $\Omega$. Assume that:

(1) $D$ is invariant under $G$,

(2) $\delta_{a}(D)=0$ for every regular element $a \in \Omega$.

Then $D T=0$ for every invariant distribution $T$ on $\Omega$.

We use induction on $\operatorname{dim} G$. By replacing $(U, V)$ in Lemma 7 with $(\Omega, \operatorname{Supp} D T)$, it becomes obvious that it would be enough to verify that no semisimple element of $\Omega$ lies in Supp DT. Let $Z$ denote the center of $G$. Fix a semisimple element $a$ in $\Omega$ and first assume that $a \notin Z$. Put $\Omega_{\Xi}=a^{-1} \Omega \cap \Xi^{\prime}$ in the notation of $\$ 4$. Then it is obvious that $\Omega_{\Xi}$ is a completely invariant open neighborhood of 1 in $\Xi$. Corresponding to Lemma 15 , we get an invariant distribution $\sigma_{T}$ on $\Omega_{\Xi}$. Moreover, $\sigma_{D T}=\Delta \sigma_{T}$, where $\Delta=\delta_{a}(D)$ (see the corollary of Lemma 15). Fix an element $b \in \Omega_{\Xi}$ which is regular in $\Xi$. Then $a b \in \Omega^{\prime}=\Omega \cap G^{\prime}$ (see the corollary of Lemma 11) and therefore

$$
\delta_{b, \Xi / A}(\Delta)=\delta_{a b}(D)=0
$$


in the notation of Lemma 12. Moreover, as we have seen in $\$ 5, \Delta$ is analytic and invariant under $\Xi$. Now $\operatorname{dim} \Xi<\operatorname{dim} G$, since $a \notin Z$. Therefore we conclude from the induction hypothesis that $\Delta \sigma_{T}=0$. But then $a \notin \operatorname{Supp} D T$ by Lemma 15 .

So now we may assume that $a \in \Omega \cap Z$. It follows from its definition (see $\S 2$ ) that the mapping $\Gamma_{x}$ depends only on $\operatorname{Ad}(x)(x \in G)$. Therefore if we apply the translation by $a^{-1}$ to the whole problem, we are reduced to the case $a=1$. So we may assume that $1 \in \Omega$ and it remains to show that $1 \notin \operatorname{Supp} D T$.

Let $c$ be the center and $g_{1}$ the derived algebra of $g$. Choose an open and relatively compact neighborhood $c_{0}$ of zero in $c$ such that the exponential mapping is univalent on $c_{0}$. Moreover, select a number $c(0<c \leqq \pi)$ and define $g_{1}(c)$ as in Lemma 8. Put $g_{0}=c_{0}+g_{1}(c)$. Then $g_{0}$ is an open and completely invariant neighborhood of zero in $\mathfrak{g}$ and the exponential mapping is everywhere regular on $\mathrm{g}_{0}$. Now suppose $\exp \left(C_{1}+X_{1}\right)=\exp \left(C_{2}+X_{2}\right)$, where $C_{i} \in \mathfrak{c}_{0}$ and $X_{i} \in \mathfrak{g}_{1}(c)$ $(i=1,2)$. Then $\exp \left(\operatorname{ad} X_{1}\right)=\exp \left(\operatorname{ad} X_{2}\right)$ and so it follows from Lemma 8 that $X_{1}=X_{2}$. Hence $\exp C_{1}=\exp C_{2}$ and therefore $C_{1}=C_{2}$ from the definition of $\mathfrak{c}_{0}$. This proves that the exponential mapping defines an analytic diffeomorphism of $g_{0}$ onto the open set $\exp g_{0}$ in $G$. Let $\log$ denote its inverse and put $U=\log \Omega_{0}$, where $\Omega_{0}=\operatorname{expg}_{0} \cap \Omega$. Let $V$ be a compact subset of $U$. Since $\mathrm{g}_{0}$ is completely invariant, $\mathrm{Cl}\left(V^{G}\right) \subset \mathrm{g}_{0}$. Moreover,

$$
\exp \left(\mathrm{Cl}\left(V^{G}\right)\right) \subset \mathrm{Cl}\left(\exp V^{G}\right)=\mathrm{Cl}\left((\exp V)^{G}\right) \subset \Omega
$$

since $\Omega$ is completely invariant. Hence it follows that $\mathrm{Cl}\left(V^{G}\right) \subset U$ and this shows that $U$ is completely invariant.

Now, in order to complete the proof, we need some preparation which will be undertaken in the next section.

10. Reduction to g. Put

$$
\xi(X)=\left|\operatorname{det}\left\{\left(e^{\operatorname{ad} X / 2}-e^{-\operatorname{ad} X / 2}\right) / \operatorname{ad} X\right\}\right|^{1 / 2} \quad(X \in \mathfrak{g}) .
$$

Then $\xi$ is analytic around every point $X_{0} \in \mathfrak{g}$, where $\xi\left(X_{0}\right) \neq 0$. Moreover, the exponential mapping of $g$ into $G$ is regular at $X_{0}$ if and only if $\xi\left(X_{0}\right) \neq 0$ (see, for example, $[5$, p. 95]).

Let $U$ be an open subset of $g$ such that the exponential mapping is regular and univalent on $U$ and put $U_{G}=\exp U$. Then $U_{G}$ is open in $G$ and the exponential mapping defines an analytic diffeomorphism of $U$ onto $U_{G}$. For any function $\phi$ on $U$, let $f_{\phi}$ denote the function on $U_{G}$ given by

$$
f_{\phi}(\exp X)=\xi(X)^{-1} \phi(X) \quad(X \in U)
$$

Then $f_{\phi}$ is $C^{\infty}$ or analytic if and only if the same holds for $\phi$. In particular, $f \rightarrow f_{\phi}$ defines a linear topological mapping of $C_{c}{ }^{\infty}(U)$ onto $C_{c}{ }^{\infty}\left(U_{G}\right)$. Moreover, it is obvious that, for any differential operator $D$ on $U_{G}$, there exists a unique dif- 
ferential operator $\Delta(D)$ on $U$ such that $D f_{\phi}=f_{\Delta(D) \phi}$ for $\phi \in C^{\infty}(U)$. Finally, $D$ is analytic if and only if $\Delta(D)$ is analytic.

As usual let $d X$ denote the Euclidean measure on $g$ and $d x$ the Haar measure on $G$. Then if $d X$ is suitably normalized, we have the relation (see [5, p. 95])

$$
d x=\xi(X)^{2} d X \quad(x=\exp X, X \in U) .
$$

Hence it follows that

$$
\int \phi_{1} \phi_{2} d X=\int f_{\phi_{1}} f_{\phi_{2}} d x
$$

for $\phi_{1} \in C^{\infty}(U)$ and $\phi_{2} \in C_{c}^{\infty}(U)$.

LEMMA 20. $\Delta\left(D^{*}\right)=\Delta(D)^{*}$ for any differential operator $D$ on $U_{G}$.

Fix $D$ and write $\Delta$ for $\Delta(D)$. Then if $\phi_{1}, \phi_{2} \in C_{c}{ }^{\infty}(U)$, we have

$$
\begin{aligned}
\int D^{*} f_{\phi_{1}} \cdot f_{\phi_{2}} d x & =\int f_{\phi_{1}} \cdot D f_{\phi_{2}} d x=\int f_{\phi_{1}} f_{\Delta \phi_{2}} d x \\
& =\int \phi_{1} \cdot \Delta \phi_{2} d X=\int \Delta^{*} \phi_{1} \cdot \phi_{2} d X \\
& =\int f_{\Delta^{*} \phi_{1}} \cdot f_{\phi_{2}} d x .
\end{aligned}
$$

This shows that $D^{*} f_{\phi_{1}}=f_{\Delta^{*} \phi_{1}}$ and from this our assertion follows immediately.

For any distribution $T$ on $U_{G}$, let $\tau_{T}$ denote the distribution on $U$ given by $\tau_{T}(\phi)=T\left(f_{\phi}\right)\left(\phi \in C_{c}{ }^{\infty}(U)\right)$. Then it follows from Lemma 20 that $\tau_{D T}=\Delta(D) \tau_{T}$.

Now assume that $U$ is invariant under $G$. Since $\exp \left(X^{x}\right)=(\exp X)^{x}(x \in G, X \in \mathfrak{g})$, $U_{G}$ is also invariant. Moreover, since $\xi$ is obviously invariant under $G$, it is clear that $\left(f_{\phi}\right)^{x}=f_{\phi^{x}}$ and $\Delta\left(D^{x}\right)=(\Delta(D))^{x}(x \in G)$ for $\phi \in C^{\infty}(U)$ and any differential operator $D$ on $U$. Similarly $\tau_{T^{x}}=\left(\tau_{T}\right)^{x}$.

11. Completion of the proof of Theorem 1. We are now ready to finish the proof of Theorem 1. Define $U$ as in $\S$ 9. Then $U_{G}=\exp U=\Omega_{0}$ and, corresponding to $T$, we get an invariant distribution $\tau_{T}$ on $U$. Since $D$ is an invariant and analytic differential operator on $U_{G}, \Delta=\Delta(D)$ is also invariant and analytic on $U$. Let $\phi$ be any invariant $C^{\infty}$-function on $U$. Then

$$
f_{\Delta \phi}=D f_{\phi}=0
$$

from Lemma 19. Hence $\Delta \phi=0$. However, since $U$ is completely invariant (see $\S 9$ ), we conclude from [4(n), Theorem 5] that $\tau_{D T}=\Delta \tau_{T}=0$. Obviously this implies that $D T=0$ on $U_{G}=\Omega_{0}$ and therefore $1 \notin \operatorname{Supp} D T$. This completes the proof of Theorem 1 .

12. Two isomorphisms. Let $m$ be a subalgebra of $g$ such that (1) $m$ is reductive in $\mathfrak{g}$ and (2) rank $\mathfrak{m}=\operatorname{rank} \mathfrak{g}$. As before, let $\mathfrak{Z}=\mathfrak{Z}(\mathfrak{g})$ be the center of $\mathfrak{G}=\mathfrak{S}\left(\mathfrak{g}_{c}\right)$ 
and $3(\mathfrak{m})$ the center of $\mathfrak{S}\left(\mathfrak{m}_{c}\right)$. We shall now define a homomorphism $\mu=\mu_{\mathfrak{g} / \mathfrak{m}}$ of 3 into $3(\mathrm{~m})$.

Fix a Cartan subalgebra $\mathfrak{h}$ of $\mathfrak{m}$. Then $\mathfrak{h}$ is also a Cartan subalgebra of $\mathfrak{g}$. Let $W$ and $W(\mathfrak{m})$ denote the Weyl groups of $(\mathfrak{g}, \mathfrak{h})$ and $(\mathfrak{m}, \mathfrak{h})$, respectively. Then $W(\mathfrak{m})$ is a subgroup of $W$. Let $I\left(\mathfrak{h}_{c}\right)$ and $I_{\mathfrak{m}}\left(\mathfrak{h}_{c}\right)$ denote the algebras of invariants of $W$ and $W(\mathfrak{m})$, respectively, in $\mathfrak{S}\left(\mathfrak{h}_{c}\right)$. Then $I_{\mathfrak{m}}\left(\mathfrak{h}_{c}\right) \supset I\left(\mathfrak{h}_{c}\right)$. Let $\gamma: \mathfrak{z} \rightarrow I\left(\mathfrak{h}_{c}\right)$ and $\gamma_{\mathfrak{m}}$ : $3(\mathfrak{m}) \rightarrow I_{\mathfrak{m}}\left(\mathfrak{h}_{c}\right)$ denote the canonical isomorphisms (see [4(e), Lemma 19]). We define $\mu(z)=\gamma_{\mathfrak{m}}^{-1}(\gamma(z))(z \in 3)$. Since any two Cartan subalgebras of $m_{c}$ are conjugate under the connected complex adjoint group of $m_{c}$, it follows easily from $[4(e), \S 6]$ that $\mu$ is independent of the choice of $\mathfrak{h}$.

LEMMA 21. $3(\mathfrak{m})$ is a free abelian module over $\mu_{\mathfrak{g} / \mathrm{m}}(3)$ of rank $[W: W(\mathfrak{m})]$.

It is enough to show that $I_{\mathfrak{m}}\left(\mathfrak{h}_{c}\right)$ is a free abelian module over $I\left(\mathfrak{h}_{c}\right)$ of rank $[W: W(\mathrm{~m})]$. The proof of this is substantially the same as that of Lemma 8 of $[4(i)]$.

If $\mathfrak{h}$ is a Cartan subalgebra of $\mathfrak{g}$, we can take $\mathfrak{m}=\mathfrak{h}$. Then it is clear that $\mu_{\mathfrak{g} / \mathfrak{h}}=\gamma$. As usual let $I\left(\mathfrak{g}_{c}\right)$ denote the algebra of all invariants of $G$ in $S\left(g_{c}\right)$. Then we have the Chevalley isomorphism $j: p \rightarrow p_{\mathfrak{h}}$ of $I\left(\mathfrak{g}_{c}\right)$ onto $\left({ }^{10}\right) I\left(\mathfrak{h}_{c}\right)$ (see [4(1), §9]). For any $z \in 3$, let $p_{z}$ denote the element $j^{-1}(\gamma(z)) \in I\left(\mathfrak{g}_{c}\right)$. Then $z \rightarrow p_{z}$ is an isomorphism of 3 onto $I\left(g_{c}\right)$. It follows again from the results of $[4(e), \S 6]$ that this isomorphism is independent of the choice of $\mathfrak{h}$. We shall call it the canonical isomorphism of 3 onto $I\left(\mathfrak{g}_{c}\right)$.

13. A consequence of Theorem 1. We use the notation of $\S 5$.

LEMMA 22. Let $U_{G}$ be a completely invariant open set in G. Fix a semisimple element $a \in U_{G}$ and define $U_{\Xi}=\Xi^{\prime} \cap\left(a^{-1} U_{G}\right)$ as in $\S 5$. Then $U_{\Xi}$ is completely invariant under $\Xi$. Let $\sigma$ be an invariant distribution on $U_{\Xi}$. Then(11)

$$
\delta_{a}(z) \sigma=\left|v_{a}\right|^{-1 / 2} \mu_{\mathfrak{g} / \mathbf{3}}(z)\left(\left|v_{a}\right|^{1 / 2} \sigma\right)
$$

for $z \in 3$.

It is obvious that $U_{\Xi}$ is an open and completely invariant subset of $\Xi$. Therefore, in view of Theorem 1 and Lemma 19, it is enough to prove the following result.

LEMMA 23. Let $V$ be an open subset of $U_{\Xi}$ and $f$ a $C^{\infty}$-function on $V$ which is locally invariant under $\Xi$. Then

$$
\delta_{a}(z) f=\left|v_{a}\right|^{-1 / 2} \mu_{\mathfrak{g} / 3}(z)\left(\left|v_{a}\right|^{1 / 2} f\right) \quad(z \in \mathcal{Z}) .
$$

Let $V^{\prime}$ be the set of those elements of $V$ which are regular in $\Xi$. Since $V^{\prime}$ is

(10) Since $\mathfrak{h}$ is abelian, we may identify $S\left(\mathfrak{h}_{c}\right)$ with $\mathfrak{S}\left(\mathfrak{h}_{c}\right)$ under the canonical mapping $\lambda$ of $S\left(\mathfrak{g}_{c}\right)$ onto $\mathfrak{G}$.

(11) Cf. [4(1), Theorem 2]. 
dense in $V$, it is enough to verify that the above equation holds on $V^{\prime}$. Fix $b \in V^{\prime}$ and $z \in 3$. Then we have to show that

$$
f\left(b ; \delta_{a}(z)\right)=f\left(b ;\left|v_{a}\right|^{-1 / 2} \mu(z) \circ\left|v_{a}\right|^{1 / 2}\right),
$$

where $\mu=\mu_{\mathfrak{g} / \mathfrak{z}}$. Let $\mathfrak{h}$ be the centralizer of $b$ in $\mathfrak{z}$ and $A$ the analytic subgroup of $G$ corresponding to $\mathfrak{h}$. Let $A^{\prime}$ denote the set of all points $h \in A$ where

$$
\operatorname{det}\left(\operatorname{Ad}(b h)^{-1}-1\right)_{\mathfrak{g} / \mathfrak{h}} \neq 0
$$

and put $U_{A}=A^{\prime} \cap b^{-1} U_{\Xi}$ and $V_{A}=\left(b^{-1} V\right) \cap U_{A}$. Then $V_{A}$ is an open neighborhood of 1 in $A^{\prime}$. Moreover,

$$
\begin{aligned}
f\left(b h ; \delta_{a}(z)\right) & =f\left(b h ; \delta_{b, \Xi / A}\left(\delta_{a}(z)\right)\right) \\
& =f\left(b h ; \delta_{a b}(z)\right) \quad\left(h \in V_{A}\right)
\end{aligned}
$$

from Lemmas 18 and 12. But since $a b$ is regular in $G$ (see the corollary of Lemma 11), it follows from Lemma 13 that

on $V_{A}$. Therefore

$$
\delta_{a b}(z)=\left|v_{a b}\right|^{-1 / 2} \gamma(z) \circ\left|v_{a b}\right|^{1 / 2}
$$

where

$$
f\left(b h ; \delta_{a}(z)\right)=\left|v_{a b}(h)\right|^{-1 / 2} F(h ; \gamma(z)),
$$

$$
F(h)=\left|v_{a b}(h)\right|^{1 / 2} f(b h) \quad\left(h \in V_{A}\right) .
$$

Now put $f_{1}(y)=\left|v_{a}(y)\right|^{1 / 2} f(y)$ for $y \in V$. Then

$$
\begin{aligned}
f\left(b h ;\left|v_{a}\right|^{-1 / 2} \mu(z) \circ\left|v_{a}\right|^{1 / 2}\right) & =\left|v_{a}(b h)\right|^{-1 / 2} f_{1}(b h ; \mu(z)) \\
& =\left|v_{a}(b h)\right|^{-1 / 2} f_{1}\left(b h ; \delta_{b, \Xi / A}(\mu(z))\right) \quad\left(h \in V_{A}\right)
\end{aligned}
$$

from Lemma 18. On the other hand it follows from Lemma 13 (applied to $\Xi$ ) and the definition of $\mu(z)$ that

$$
\delta_{b, \Xi / A}(\mu(z))=\left|v_{b, \Xi}\right|^{-1 / 2} \gamma(z) \circ\left|v_{b, \Xi}\right|^{1 / 2}
$$

on $U_{A}$, where

Therefore

$$
v_{b, \Xi}(h)=\operatorname{det}\left(\operatorname{Ad}(b h)^{-1}-1\right)_{z / \mathfrak{h}} \quad(h \in A) .
$$

$$
f_{1}\left(b h ; \delta_{b, \Xi / A}(\mu(z))\right)=\left|v_{b . \Xi}(h)\right|^{-1 / 2} F_{1}(h ; \gamma(z)),
$$

where

$$
F_{1}(h)=\left|v_{b, \Xi}(h)\right|^{1 / 2} f_{1}(b h)=\left|v_{b, \Xi}(h) v_{a}(b h)\right|^{1 / 2} f(b h) \quad\left(h \in V_{A}\right)
$$

But since

$$
v_{b, \Xi}(h) v_{a}(b h)=v_{a b}(h),
$$

we have $F=F_{1}$. This shows that 


$$
\begin{aligned}
f\left(b h ;\left|v_{a}\right|^{-1 / 2} \mu(z) \circ\left|v_{a}\right|^{1 / 2}\right) & =\left|v_{a}(b h) v_{b, \Xi}(h)\right|^{-1 / 2} F_{1}(h ; \gamma(z)) \\
& =\left|v_{a b}(h)\right|^{-1 / 2} F(h ; \gamma(z))=f\left(b h ; \delta_{a}(z)\right)
\end{aligned}
$$

for $h \in V_{A}$. Putting $h=1$, we get the required result.

14. The relation between 3 and $\partial\left(I\left(\mathfrak{g}_{c}\right)\right)$. We now use the notation of $\$ 10$. For any open subset $V$ of $U$ and a function $\phi$ on $V$, we define, as before, the function $f_{\phi}$ on $V_{G}=\exp V$ by $f_{\phi}(\exp X)=\xi(X)^{-1} \phi(X)(X \in V)$. Let $z \rightarrow p_{z}$ denote the canonical isomorphism of 3 onto $I\left(\mathfrak{g}_{c}\right)$ (see $\S 12$ ).

LEMMA 24. Let $\phi$ be a locally invariant $C^{\infty}$-function on an open subset $V$ of $U$. Then

for $z \in \mathcal{3}$.

$$
z f_{\phi}=f_{\partial\left(p_{z}\right) \phi}
$$

Fix $z \in 3$ and let $V^{\prime}$ be the set of all regular points in $V$. Consider the differential operator $\Delta(z)$ on $U$ corresponding to $z$ (see $\$ 12)$. Then it is enough to prove that $\Delta(z) \phi=\partial\left(p_{z}\right) \phi$ on $V^{\prime}$. Fix a point $H_{0} \in V^{\prime}$ and let $\mathfrak{h}$ denote the centralizer of $H_{0}$ in $\mathfrak{g}$. Then $\mathfrak{h}$ is a Cartan subalgebra of $\mathfrak{g}$. Let $\mathfrak{h}_{0}$ be an open and connected neighborhood of $H_{0}$ in $\mathfrak{h} \cap V^{\prime}$. Then it would be sufficient to show that

$$
\phi(H ; \Delta(z))=\phi\left(H ; \partial\left(p_{z}\right)\right) \quad\left(H \in \mathfrak{h}_{0}\right) .
$$

Since $\phi$ is locally invariant, it follows from [4(1), Lemma 14] and [4(f), Theorem 1] that

$$
\phi\left(H ; \partial\left(p_{z}\right)\right)=\phi\left(H ; \delta_{\mathfrak{g} / \mathfrak{h}}{ }^{\prime}\left(\partial\left(p_{z}\right)\right)\right)=\pi(H)^{-1} \phi(H ; \partial(q) \circ \pi) \quad\left(H \in \mathfrak{h}_{0}\right) .
$$

Here $\pi$ denotes, as usual, the product of all the positive roots of $(\mathfrak{g}, \mathfrak{h})$ and $q=\left(p_{z}\right)_{\mathfrak{h}}$ in the notation of [4(1), §8]. Let $A$ be the analytic subgroup of $G$ corresponding to $\mathfrak{h}$ and put $A^{\prime}=A \cap G^{\prime}$ and

$$
v(h)=\operatorname{det}\left(\operatorname{Ad}(h)^{-1}-1\right)_{\mathfrak{g} / \mathfrak{b}} \quad(h \in A) .
$$

Since $\mathfrak{h}_{0} \subset U$, it follows that $\xi(H) \neq 0$ and therefore $\exp H \in A^{\prime}$ for $H \in \mathfrak{h}_{0}$. Moreover, it is clear that $f_{\phi}$ is locally invariant with respect to $G$. Therefore we conclude from Lemma 17 and [4(e), Theorem 2] that

$$
f_{\phi}(\exp H ; z)=|v(\exp H)|^{-1 / 2} f_{\phi}\left(\exp H ; \gamma(z) \circ|v|^{1 / 2}\right)
$$

for $H \in \mathfrak{h}_{0}$. But it is obvious that

and therefore

$$
|v(\exp H)|^{1 / 2}=\xi(H)|\pi(H)|
$$

$$
|v(\exp H)|^{1 / 2} f_{\phi}(\exp H)=|\pi(H)| \phi(H) \quad\left(H \in \mathfrak{h}_{0}\right) .
$$

If $r$ is the number of positive roots of $(\mathfrak{g}, \mathfrak{h})$, we know that $\operatorname{det}(\operatorname{ad} H)=(-1)^{r} \pi(H)^{2}$ $(H \in \mathfrak{h})$. This shows that $\pi(H)^{2}$ is real. Therefore since $\mathfrak{h}_{0}$ is connected and $\pi$ is 
nowhere zero on $\mathfrak{h}_{0}$, it follows that $|\pi(H)|=\varepsilon \pi(H)\left(H \in \mathfrak{h}_{0}\right)$, where $\varepsilon=\left|\pi\left(H_{0}\right)\right| / \pi\left(H_{0}\right)$ Moreover, $j\left(p_{z}\right)=\gamma(z)$ in the notation of $\$ 12$. Hence it is clear that

$$
\begin{aligned}
f_{\phi}(\exp H ; z) & =\pi(H)^{-1} \xi(H)^{-1} \phi(H ; \partial(q) \circ \pi) \\
& =\xi(H)^{-1} \phi\left(H ; \partial\left(p_{z}\right)\right) \quad\left(H \in \phi_{0}\right) .
\end{aligned}
$$

On the other hand

$$
\phi(X ; \Delta(z))=\xi(X) f_{\phi}(\exp X ; z) \quad(X \in V)
$$

from the definition of $\Delta(z)$. Therefore

$$
\phi(H ; \Delta(z))=\phi\left(H ; \partial\left(p_{z}\right)\right) \quad\left(H \in \mathfrak{h}_{0}\right)
$$

and this proves the lemma.

Corollary. Assume that $U$ is completely invariant. Then if $T$ is an invariant distribution on $U_{G}$,

$$
\tau_{z T}=\partial\left(p_{z}\right) \tau_{T} \quad(z \in 3) .
$$

We know (see $\S 10$ ) that $\tau_{z T}=\Delta(z) \tau_{T}$ and it follows from Lemma 24 and [4(n), Theorem 5] that $\Delta(z) \tau_{T}=\partial\left(p_{z}\right) \tau_{T}$. Hence the corollary.

15. Proof of Theorem 2. We now come to one of the main results of this paper (cf. [4(j), Theorem 1]).

THEOREM 2. Let $\Omega$ be a completely invariant open set in $G$ and $T$ a distribution on $\Omega$. We assume that:

(1) $T$ is invariant;

(2) there exists an ideal $\mathfrak{U}$ in 3 such that $\operatorname{dim} \mathfrak{Z} / \mathfrak{U}<\infty$ and $u T=0$ for $u \in \mathfrak{U}$. Then $T$ is a locally summable function which is analytic on $\Omega^{\prime}=\Omega \cap G^{\prime}$.

We shall use induction on $\operatorname{dim} G$. Let $\Omega_{0}$ be the set of all points $a \in \Omega$ with the following property. There exists an open neighborhood $U$ of $a$ in $\Omega$ and a locally summable function $F$ on $U$ such that $F$ is analytic on $U \cap G^{\prime}$ and $T=F$ on $U$. Clearly $\Omega_{0}$ is an open and invariant subset of $\Omega$. It would be enough to prove that $\Omega_{0}=\Omega$. But then, in view of Lemma 7 , we have only to verify that $\Omega_{0}$ contains all semisimple points of $\Omega$.

LEMma 25. $\Omega^{\prime} \subset \Omega_{0}$.

Fix $a \in \Omega^{\prime}$ and let $\mathfrak{h}$ denote the centralizer of $a$ in $\mathfrak{g}$. Then $\mathfrak{h}$ is a Cartan subalgebra of $\mathfrak{g}$. Consider the analytic subgroup $A$ of $G$ corresponding to $\mathfrak{h}$ and put $\Omega_{A}=a^{-1} \Omega \cap A^{\prime}$, where $A^{\prime}$ is the set of all $h \in A$ such that

$$
v_{a}(h)=\operatorname{det}\left(\operatorname{Ad}(a h)^{-1}-1\right)_{\mathfrak{g} / \mathfrak{h}} \neq 0 .
$$

Let $\sigma_{T}$ denote the distribution on $\Omega_{A}$ corresponding to $T$ under Lemma 15. Put $\sigma=\left|v_{a}\right|^{1 / 2} \sigma_{T}$. Then we conclude from Lemma 13 and the corollary of Lemma 15 
that $\gamma(u) \sigma=0$ for $u \in \mathfrak{U}$. Since $\mathfrak{S}\left(\mathfrak{h}_{c}\right)$ is a finite module over $\gamma(\mathfrak{Z})=I\left(\mathfrak{h}_{c}\right)$ (Lemma 21), it follows that $\mathfrak{B}=\mathfrak{S}\left(\mathfrak{h}_{c}\right) \gamma(\mathfrak{U})$ has finite codimension in $\mathfrak{S}\left(\mathfrak{h}_{c}\right)$. Fix a base $H_{1}, \cdots, H_{l}$ for $\mathfrak{h}$ over $\boldsymbol{R}$ and put

$$
\square=H_{1}^{2}+\cdots+H_{l}^{2} .
$$

Then if $N=\operatorname{dim} \subseteq\left(\mathfrak{h}_{c}\right) / \mathfrak{B}$, it is obvious that we can choose $c_{i} \in C(1 \leqq i \leqq N)$ such that

$$
v=\square^{N}+\sum_{1 \leqq k \leqq N} c_{k} \square^{N-k} \in \mathfrak{B} .
$$

Now $v$ is an analytic differential operator on $A$ which is obviously elliptic. Therefore since $v \sigma=0$, we conclude that $\sigma$ coincides with an analytic function $g$ on $\Omega_{A}$. Put $G^{*}=G / A$ and define the mapping $\psi: G^{*} \times \Omega_{A} \rightarrow \Omega$ as in the proof of Lemma 19 . Then we can choose open neighborhoods $G_{0}$ and $V$ of 1 in $G$ and $\Omega_{A}$, respectively, such that $\psi$ defines an analytic diffeomorphism of $G_{0}{ }^{*} \times V$ onto the open subset $U=\psi\left(G_{0}^{*} \times V\right)$ of $\Omega$. Define the analytic function $F$ on $U$ by

$$
F\left((a h)^{x^{*}}\right)=\left|v_{a}(h)\right|^{-1 / 2} g(h) \quad\left(x^{*} \in G_{0}^{*}, h \in V\right) .
$$

Then by Lemma 15, we get

$$
T\left(f_{\alpha}\right)=\sigma_{T}\left(\beta_{\alpha}\right)=\int \beta_{\alpha}\left|v_{a}\right|^{-1 / 2} g d h \quad\left(\alpha \in C_{c}{ }^{\infty}\left(G_{0} \times V\right)\right),
$$

where $d h$ is the Haar measure on $A$. On the other hand, it follows from the definition of $f_{\alpha}$ that

$$
\begin{aligned}
\int f_{\alpha} F d x & =\int \alpha(x: h) F\left((a h)^{x}\right) d x d h \\
& =\int \beta_{\alpha}\left|v_{a}\right|^{-1 / 2} g d h .
\end{aligned}
$$

This shows that $T=F$ on $U$ and therefore $a \in \Omega_{0}$.

It is clear from the above lemma that there exists an analytic function $F$ on $\Omega^{\prime}$ such that $T=F$ on $\Omega^{\prime}$. Now fix a semisimple element $a \in \Omega$ and let us use the notation of $\S 4$. $Z$ being the center of $G$, first assume that $a \notin Z$ so that $\operatorname{dim} z<\operatorname{dim} g$. Put $\Omega_{\Xi}=a^{-1} \Omega \cap \Xi^{\prime}$. Then $\Omega_{\Xi}$ is an open and completely invariant neighborhood of 1 in $\Xi$. Let $\sigma_{T}$ denote the distribution on $\Omega_{\Xi}$ which corresponds to $T$ under Lemma 15. Then $\sigma_{T}$ is invariant under $\Xi$ and it follows from the corollary of Lemma 15 that $\delta_{a}(u) \sigma_{T}=0$ for $u \in \mathfrak{U}$. But then we conclude from Lemma 22 that

$$
\mu(u) \sigma=0 \quad(u \in \mathfrak{U}) .
$$

Here $\sigma=\left|v_{a}\right|^{1 / 2} \sigma_{T}$ and $\mu=\mu_{\mathfrak{g} / \mathfrak{3}}$. Since $3(\mathfrak{Z})$ is a finite module over $\mu(3)$ (Lemma 21), it is clear that $\mathfrak{B}=\mathfrak{Z}(\mathfrak{z}) \mu(\mathfrak{U})$ has finite codimension in $3(\mathfrak{z})$. Let $\Omega_{\Xi}{ }^{\prime}$ be the set of those elements in $\Omega_{\Xi}$ which are regular in $\Xi$. Then it follows by the induction hypothesis that $\sigma=g$, where $g$ is a locally summable function on $\Omega_{\Xi}$ which is analytic on $\Omega_{\Xi}{ }^{\prime}$. 
Let $\phi$ denote the mapping $(x, y) \rightarrow(a y)^{x}$ of $G \times \Omega_{\Xi}$ into $\Omega$. Then $U=\phi\left(G \times \Omega_{\Xi}\right)$ is an open neighborhood of $a$ in $\Omega$ (Lemma 14). Moreover, it is easy to verify that $\phi\left(G \times \Omega_{\Xi}{ }^{\prime}\right)=U^{\prime}$, where $U^{\prime}=U \cap G^{\prime}=U \cap \Omega^{\prime}$. Since $T=F$ on $\Omega^{\prime}$, we have

$$
T\left(f_{\alpha}\right)=\int f_{\alpha} F d x=\int \alpha(x: y) F\left((a y)^{x}\right) d x d y
$$

for $\alpha \in C_{c}{ }^{\infty}\left(G \times \Omega_{\Xi}{ }^{\prime}\right)$ in the notation of Lemma 15 . However,

$$
T\left(f_{\alpha}\right)=\sigma_{T}\left(\beta_{\alpha}\right)=\int \alpha(x: y)\left|v_{\alpha}(y)\right|^{-1 / 2} g(y) d x d y .
$$

This shows that the analytic function

$$
(x, y) \rightarrow F\left((a y)^{x}\right)-\left|v_{a}(y)\right|^{-1 / 2} g(y)
$$

is zero on $G \times \Omega_{\Xi}{ }^{\prime}$ and therefore $F \circ \phi$ is locally summable on $G \times \Omega_{\Xi}$. Hence $F$ is locally summable on $U$ (see [4(k), Corollary 2 of Theorem 1]) and

$$
\begin{aligned}
\int f_{\alpha} F d x & =\int \alpha(x: y) F\left((a y)^{x}\right) d x d y \\
& =\int \alpha(x: y)\left|v_{a}(y)\right|^{-1 / 2} g(y) d x d y \\
& =\sigma_{T}\left(\beta_{\alpha}\right)=T\left(f_{\alpha}\right)
\end{aligned}
$$

for $\alpha \in C_{c}{ }^{\infty}\left(G \times \Omega_{\Xi}\right)$. This proves that $T=F$ on $U$ and therefore $a \in \Omega_{0}$.

It remains to consider the case when $a \in Z$. Then by a translation by $a^{-1}$, we are reduced to the case $a=1$. Then, as we have seen in $\$ 9$, there exists an open and completely invariant neighborhood $U$ of zero in $\mathfrak{g}$ such that the exponential mapping of $g$ into $G$ is univalent and regular on $U$ and $U_{G}=\exp U \subset \Omega$. Let $\tau_{T}$ be the distribution on $U$ corresponding to $T$ (see $\S 10$ ). Then we know from the corollary of Lemma 24 that $\partial\left(p_{u}\right) \tau_{T}=0$ for $u \in \mathfrak{U}$. Let $\mathfrak{B}$ denote the image of $\mathfrak{U}$ in $I\left(\mathfrak{g}_{c}\right)$ under the canonical isomorphism $z \rightarrow p_{z}$ of $\mathcal{Z}$ onto $I\left(\mathfrak{g}_{c}\right)$. Then

$$
\operatorname{dim} I\left(\mathfrak{g}_{c}\right) / \mathfrak{B}=\operatorname{dim} \mathfrak{3} / \mathfrak{U}<\infty
$$

and so we conclude from [4(n), Theorem 1] that $\tau_{T}=\Phi$, where $\Phi$ is a locally summable function on $U$. Define the function $f_{\Phi}$ on $U_{G}$ as in $\S 10$. Then it is obvious that $f_{\Phi}$ is locally summable on $U_{G}$ and $T=f_{\Phi}$ on $U_{G}$. But since $T=F$ on $U_{G} \cap \Omega^{\prime}$, it follows that $f_{\Phi}=F$ almost everywhere on $U_{G}$. Hence $F$ is locally summable on $U_{G}$ and $T=F$ on $U_{G}$. This shows that $1 \in \Omega_{0}$ and so the proof of Theorem 2 is now complete.

The above theorem shows that $F$ is locally summable on $\Omega$ and $T=F$ on $\Omega$. Fix $z \in 3$. Then the distribution $z T$ also satisfies all the conditions of Theorem 2 and it is obvious that $z T=z F$ on $\Omega^{\prime}$. Hence $z F$ is also locally summable on $\Omega$ and $z T=z F$ on $\Omega$. Thus we obtain the following corollary (cf. [4(n), Lemma 16]). 
CoRollary. For any $z \in 3$, the function $z F$ on $\Omega^{\prime}$ is locally summable on $\Omega$ and $z T=z F$. Hence

$$
\int f \cdot z F d x=\int z^{*} f \cdot F d x
$$

for $f \in C_{c}^{\infty}(\Omega)$.

16. Some elementary facts about reductive groups. As before let $\mathrm{c}$ be the center and $\mathfrak{g}_{1}$ the derived algebra of $\mathfrak{g}$. Fix a Cartan subalgebra $\mathfrak{h}$ of $\mathfrak{g}$. Then $\mathfrak{h}_{1}=\mathfrak{h} \cap \mathfrak{g}_{1}$ is a Cartan subalgebra of the semisimple Lie algebra $\mathfrak{g}_{1}$. We can choose a Cartan involution $\theta$ of $\mathfrak{g}_{1}$ such that $\theta\left(\mathfrak{h}_{1}\right)=\mathfrak{h}_{1}$ [4(e), p. 100]. We extend $\theta$ to an automorphism of $\mathfrak{g}$ by defining $\theta(C)=C$ for $C \in \mathcal{C}$. Let $\mathfrak{l}$ and $\mathfrak{p}$ be the subspaces of $\mathfrak{g}$ corresponding to the eigenvalues 1 and -1 of $\theta$. Then $c \subset \mathfrak{f}$ and $p \subset g_{1}$. Moreover, since $\theta(\mathfrak{h})=\mathfrak{h}$, it is clear that $\mathfrak{h}=\mathfrak{h} \cap \mathfrak{t}+\mathfrak{h} \cap \mathfrak{p}$.

Let $K$ be the analytic subgroup of $G$ corresponding to $\mathfrak{f}$ and $Z$ the center of $G$.

LemMa 26. The mapping $\phi:(k, X) \rightarrow k \exp X(k \in K, X \in \mathfrak{p})$ is an analytic diffeomorphism of $K \times \mathfrak{p}$ onto $G$. Moreover, $Z \subset K$ and $K / Z$ is compact.

It is easy to verify (see [4(d), p. 614]) that $\phi$ is everywhere regular. Let $C, G_{1}$ and $K_{1}$ be the analytic subgroups of $G$ corresponding to $\mathfrak{c}, \mathfrak{g}_{1}$ and $\mathfrak{f}_{1}=\mathfrak{f} \cap \mathfrak{g}_{1}$, respectively. Then $G=C G_{1}$ and $G_{1}=K_{1} \operatorname{expp~(see~e.g.~[5,~pp.~214-215]).~There-~}$ fore since $C K_{1}=K$, it follows that $\phi$ is surjective. Now suppose

$$
k_{1} \exp X_{1}=k_{2} \exp X_{2} \quad\left(k_{i} \in K, X_{i} \in \mathfrak{p}, i=1,2\right) .
$$

Put $k=k_{2}{ }^{-1} k_{1}$. Then $k \exp X_{1}=\exp X_{2}$ and therefore

$$
\operatorname{Ad}(k) \exp \left(\operatorname{ad} X_{1}\right)=\exp \left(\operatorname{ad} X_{2}\right) .
$$

Since $\operatorname{Ad}(G)$ is semisimple, we conclude [5, pp. 214-215] that $X_{1}=X_{2}$. Hence $k_{1}=k_{2}$. This proves that $\phi$ is univalent and so it is an analytic diffeomorphism.

Let $Z_{1}$ be the center of $G_{1}$. Then we know that $Z_{1} \subset K_{1}$ and $K_{1} / Z_{1}$ is compact [5,p.214]. Since $K=C K_{1}$ and $Z=C Z_{1}$, it follows that $Z \subset K$ and $K / Z$ is compact.

COROLlary. 1. $\theta$ can be extended to an automorphism of $G$ such that

$$
\theta(k \exp X)=k \exp (-X) \quad(k \in K, X \in \mathfrak{p}) .
$$

First assume that $G$ is simply connected. Then our statement is obvious. Moreover, $\theta$ leaves $Z$ pointwise fixed since $Z \subset K$. Therefore if $Z_{0}$ is any closed subgroup of $Z$, it defines an automorphism of $G / Z_{0}$. From this our assertion follows immediately in the general case.

Corollary 2(12). Let $Y^{\prime}=\operatorname{Ad}(k \exp X) Y$, where $Y, Y^{\prime} \in \mathfrak{g}, k \in K$ and $X \in \mathfrak{p}$. Then if $Y$ and $Y^{\prime}$ are both eigenvectors of $\theta,[X, Y]=0$.

(12) This result was pointed out to me by A. Borel. 
Since

$$
\theta\left(Y^{\prime}\right)=\operatorname{Ad}(k \exp (-X)) \theta(Y)
$$

it is clear that

$$
e^{2 \mathrm{adX}} Y=\varepsilon Y,
$$

where $\varepsilon= \pm 1$. Moreover, it follows from [4(k), Lemma 27] that ad $X$ is semisimple and all its eigenvalues are real. Therefore it is obvious that $\varepsilon=1$ and $[X, Y]=0$.

COROLlary 3. Let $\mathfrak{a}$ be a subset of $\mathfrak{h}$ such that $\mathfrak{a}=\theta(\mathfrak{a})$ and let $\Xi$ and $\mathfrak{z}$ be the centralizers of $\mathfrak{a}$ in $G$ and $\mathfrak{g}$, respectively. Then they are both invariant under $\theta$, 3 is reductive in $\mathrm{g}$ and

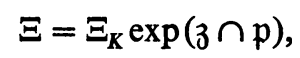

where $\Xi_{K}=\Xi \cap K$.

For the proof we can obviously replace $\mathfrak{a}$ by the linear subspace of $\mathfrak{g}$ spanned by it. Then $\mathfrak{a}=\mathfrak{a} \cap \mathfrak{f}+\mathfrak{a} \cap \mathfrak{p}$. The invariance of $\Xi$ and $\mathfrak{z}$ under $\theta$ is obvious and therefore (see $[4(\mathrm{~g})$, Lemma 10$]) z$ is reductive in $\mathfrak{g}$. The last statement follows from Corollary 1.

Let $A$ be the Cartan subgroup of $G$ corresponding to $\mathfrak{h}$.

Corollary 4. $A=A_{K} A_{\mathfrak{p}}$, where $A_{K}=A \cap K$ and $A_{\mathfrak{p}}=\exp (\mathfrak{h} \cap \mathfrak{p})$. Moreover, $Z \subset A_{K}$ and $A_{K} / Z$ is compact.

The first statement follows from Corollary 3 if we take $\mathfrak{a}=\mathfrak{h}$. It is obvious from Lemma 26 that $Z \subset A_{K}$. Moreover, since $K / Z$ is compact and $A_{K}$ is closed in $K$, it follows that $A_{K} / Z$ is compact.

COROllary 5. Suppose every root of $(\mathfrak{g}, \mathfrak{h})$ is imaginary $($ see $[4(\mathrm{~m}), \S 4])$. Then $A$ is connected and contained in $K$.

For then it is obvious that $\mathfrak{h} \cap \mathfrak{p}=\{0\}$ and therefore $A=A_{K}$. Since $\mathfrak{f}$ is reductive and its derived algebra is compact, the connected component of 1 in $A$ is maximal abelian in $K$. This shows that $A$ is connected.

17. Complex semisimple groups. Let $\mathfrak{g}_{c}$ be a complex semisimple Lie algebra and $G_{c}$ a complex analytic group corresponding to it. Fix a Cartan subalgebra $\mathfrak{h}_{c}$ of $\mathfrak{g}_{c}$. Then we can choose a compact real form $\mathfrak{u}$ of $\mathfrak{g}_{c}$ such that $\mathfrak{h}=\mathfrak{h}_{c} \cap \mathfrak{u}$ is a Cartan subalgebra of $\mathfrak{u}$ (see [5, p. 155]). Let $\eta$ denote the conjugation of $\mathfrak{g}_{c}$ with respect to $\mathfrak{u}$. Then if we regard $\mathfrak{g}_{c}$ as a Lie algebra over $\boldsymbol{R}, \boldsymbol{\eta}$ is a Cartan involution of $\mathfrak{g}_{c}$ and $\mathfrak{g}_{c}=\mathfrak{u}+(-1)^{1 / 2} \mathfrak{u}$ is the corresponding Cartan decomposition. Let $U$ be the real analytic subgroup of $G_{c}$ corresponding to $\mathfrak{u}$. Then $U$ is compact and by Lemma 26, the mapping

$$
(u, X) \rightarrow u \exp (-1)^{1 / 2} X \quad(u \in U, X \in \mathfrak{u})
$$

is an analytic diffeomorphism of $U \times \mathfrak{u}$ onto $G_{c}$. 
LEMMA 27. Let $\mathfrak{a}$ be a subset of $\mathfrak{h}_{c}$ such that $\eta(\mathfrak{a})=\mathfrak{a}$ and let $z_{c}$ and $\Xi_{c}$ denote the centralizers of $\mathfrak{a}$ in $\mathfrak{g}_{c}$ and $G_{c}$, respectively. Then $\mathfrak{z}_{c}$ is reductive in $\mathfrak{g}_{c}$ and $\Xi_{c}$ is connected.

We may obviously replace $\mathfrak{a}$ by the subspace $\mathfrak{a}_{c}$ spanned by it over $\boldsymbol{C}$. It follows from Corollary 3 of Lemma 26 that $z_{c}$ is reductive in $g_{c}$ and

$$
\left.\Xi_{c}=\Xi \exp \left((-1)^{1 / 2}\right\}\right)
$$

where $\Xi=\Xi_{c} \cap U$ and $\mathfrak{z}=\mathfrak{z}_{c} \cap \mathfrak{u}$. It is clear that $\Xi$ is the centralizer of $\mathfrak{a}_{c} \cap \mathfrak{u}$ in $U$ and therefore it is connected (see [5, p. 247]). This proves that $\Xi_{c}$ is connected.

Corollary. Let $A_{c}$ be the Cartan subgroup of $G_{c}$ corresponding to $\mathfrak{h}_{c}$. Then $A_{c}$ is connected.

By definition $A_{c}$ is the centralizer of $\mathfrak{h}_{c}$ in $G_{c}$. Hence the corollary follows by taking $\mathfrak{a}=\mathfrak{h}_{c}$.

The following lemma, together with its proof, was pointed out to me by Borel.

LEMMA 28. $\jmath_{c}$ being as above, put ${z_{1 c}}_{1 c}\left[3_{c}, 3_{c}\right]$ and let $\Xi_{1 c}$ be the complex analytic subgroup of $G_{c}$ corresponding to $3_{1 c}$. Then if $G_{c}$ is simply connected, the same holds for $\Xi_{1 \mathrm{c}}$.

Put $\mathfrak{h}_{R}=(-1)^{1 / 2}\left(\mathfrak{h}_{c} \cap \mathfrak{u}\right)$ and $\mathfrak{a}_{R}=(-1)^{1 / 2}\left(\mathfrak{a}_{c} \cap \mathfrak{u}\right)$. Introduce compatible orders (see $\left[4(\mathrm{~g})\right.$, p. 195]) in the spaces of linear functions on $\mathfrak{h}_{R}$ and $\mathfrak{a}_{R}$. Let $P$ be the set of all positive roots of $\left(\mathfrak{g}_{c}, \mathfrak{h}_{c}\right)$ under this order. Let $\bar{\alpha}$ denote the restriction of $\alpha$ on $\mathfrak{a}_{c}$ for any root $\alpha$ and let $P_{0}$ denote the set of those $\alpha \in P$ for which $\bar{\alpha}=0$. Consider the set $\left(\alpha_{1}, \alpha_{2}, \cdots, \alpha_{l}\right)$ of simple roots in $P$ and assume that $\alpha_{i} \in P_{0}$ $(1 \leqq i \leqq m)$ and $\alpha_{i} \notin P_{0}(m<i \leqq l)$. We claim that $\left(\alpha_{1}, \cdots, \alpha_{m}\right)$ is a set of fundamental roots for $\left(\mathfrak{z}_{c}, \mathfrak{h}_{c}\right)$. Fix $\alpha \in P_{0}$. Then $\alpha=\Sigma_{1 \leqq i \leqq l} r_{i} \alpha_{i}$, where $r_{i}$ are integers $\geqq 0$. Hence

$$
\sum_{1 \leqq i \leqq l} r_{i} \bar{\alpha}_{i}=\bar{\alpha}=0 .
$$

Now $\bar{\alpha}_{i}=0(1 \leqq i \leqq m)$ and $\bar{\alpha}_{i}>0(m<i \leqq l)$ by the compatibility of our orders. So it is obvious that $r_{i}=0$ for $m<i \leqq l$. Since $\left(\alpha_{1}, \cdots, \alpha_{m}\right)$ are linearly independent, this proves our assertion.

For any root $\alpha$, let $H_{\alpha}$ denote, as usual, the element in $\mathfrak{h}_{R}$ such that $\operatorname{tr}\left(\operatorname{ad} H \operatorname{ad} H_{\alpha}\right)=\alpha(H)$ for $H \in \mathfrak{h}_{c}$. Put

$$
H_{i}=2 \alpha_{i}\left(H_{\alpha_{i}}\right)^{-1} H_{\alpha_{i}} \quad(1 \leqq i \leqq l) .
$$

Then it is clear that $H_{i}(1 \leqq i \leqq m)$ form a base for $\mathfrak{h}_{c} \cap \mathfrak{z}_{1 c}$ over $C$. Now suppose $t_{i}(1 \leqq i \leqq l)$ are complex numbers such that

$$
\exp \left(2 \pi(-1)^{1 / 2} \sum_{1 \leqq i \leqq m} t_{i} H_{i}\right)=1
$$


in $\Xi_{1 c}$. Then since $G_{c}$ is simply connected, we can conclude (see Weyl [8]) that $t_{i}$ are rational integers. This implies that $\Xi_{1 c}$ is simply connected.

LEMma 29. Assume that $G_{c}$ is simply connected and let $\lambda$ be a linear function on $\mathfrak{h}_{c}$. Then there exists a character $\xi_{\lambda}$ of $A_{c}$ such that

$$
\xi_{\lambda}(\exp H)=e^{\lambda(H)} \quad\left(H \in \mathfrak{h}_{c}\right)
$$

if and only if $2 \lambda\left(H_{\alpha}\right) / \alpha\left(H_{\alpha}\right)$ is a rational integer for every root $\alpha$. Put

$$
\rho=\frac{1}{2} \sum_{\alpha \in P} \alpha
$$

where $P$ is the set of positive roots under some order. Then the above condition is fulfilled for $\lambda=\rho$.

This is well known (see Weyl [8]).

18. Acceptable groups. Let $G$ be a connected Lie group with the Lie algebra $\mathrm{g}$ over $\boldsymbol{R}$ which we assume, as before, to be reductive. Let $j$ be the inclusion mapping of $\mathfrak{g}$ into $\mathfrak{g}_{c}$ and $G_{c}$ a complex analytic group corresponding to $\mathfrak{g}_{c}$. We say that $G_{c}$ is a complexification of $G$ if $j$ can be extended to a homomorphism of $G$ into $G_{c}$.

Define $\mathfrak{c}$ and $\mathfrak{g}_{1}$ as in $\$ 9$ and let $C$ and $G_{1}$, respectively, be the corresponding analytic subgroups of $G$. We call $G_{1}$ the semisimple part of $G$. Similarly let $C_{c}$ and $G_{1 c}$ denote the complex analytic subgroups of $G_{c}$ corresponding to $\mathcal{C}_{c}$ and $\mathrm{g}_{1 c}$, respectively. We say that $G_{c}$ is quasisimply connected (q. s. c.) if $C_{c} \cap G_{1 c}=\{1\}$ and $G_{1 c}$ is simply connected. Moreover, $G$ itself is called q. s. c. if it has a q. s. c. complexification. Assume that $G_{1} \cap C$ is finite. Then $G$ always has a complexification. Moreover, since the center of a complex semisimple group is finite, it is clear that there exists a q. s. c. covering group $\tilde{G}$ which covers $G$ only finitely many times.

Let $A$ be the Cartan subgroup of $G$ corresponding to $\mathfrak{h}$. Consider a complexification $G_{c}$ of $G$ and let $A_{c}$ denote its Cartan subgroup corresponding to $\mathfrak{h}_{c}$. Then $A_{c}$ is connected (corollary of Lemma 27) and it is obvious that $j(A) \subset A_{c}$. Let $\lambda$ be a linear function on $\mathfrak{h}_{c}$. Then there exsts at most one complex-analytic homomorphism $\xi_{\lambda}$ of $A_{c}$ into $C$ such that

$$
\xi_{\lambda}(\exp H)=e^{\lambda(\boldsymbol{H})} \quad\left(H \in \mathfrak{h}_{c}\right) .
$$

Then $\xi_{\lambda} \circ j$ is a homomorphism of $A$ into $C$, which is easily seen to be independent of the particular choice of $G_{c}$ (so long as it can be defined by means of $G_{c}$ at all). We shall write $\xi_{\lambda}$ instead of $\xi_{\lambda} \circ j$. If $\alpha$ is a root of $(\mathfrak{g}, \mathfrak{h})$, it is obvious that $\xi_{\alpha}$ always exists.

Let $P$ be the set of all positive roots of $(\mathfrak{g}, \mathfrak{h})$ in some order and put

$$
\rho=\frac{1}{2} \sum_{\alpha \in P} \alpha
$$

If $G$ is q. s. c., we can take $G_{c}$ to be a q. s. c. complexification of $G$. Then it follows from Lemma 29 that $\xi_{\rho}$ exists. 
Let $W$ denote the Weyl group of $(\mathfrak{g}, \mathfrak{h})$. Then it is well known (see [8]) that $s \rho-\rho(s \in W)$ is an integral linear combination of the roots. Therefore the condition that $\xi_{\rho}$ should be defined is independent of the order of roots. Moreover, since any two Cartan subalgebras of $g_{c}$ are conjugate under the (connected) adjoint group of $\mathfrak{g}_{c}$, it follows that the above condition also does not depend on the choice of $\mathfrak{h}$. We shall say that $G$ is acceptable if this condition is satisfied. Similarly a complexification $G_{c}$ of $G$ is called acceptable if $\xi_{\rho}$ can be defined on $A_{c}$.

Let $m$ be the centralizer of $\mathfrak{h} \cap \mathfrak{p}$ in $\mathfrak{g}$ and $M$ the analytic subgroup of $G$ corresponding to $\mathrm{m}$. Introduce compatible orders (see $[4(\mathrm{~g}), \mathrm{p} .195])$ on the spaces of real linear functions on $\mathfrak{h} \cap \mathfrak{p}$ and $\mathfrak{h} \cap \mathfrak{p}+(-1)^{1 / 2} \mathfrak{h} \cap \mathfrak{f}$ respectively. We assume that $P$ is the set of positive roots under this order. Let $P_{M}$ denote the set of those $\alpha \in P$ which vanish identically on $\mathfrak{h} \cap \mathfrak{p}$. Put

$$
\rho_{M}=\frac{1}{2} \sum_{\alpha \in P_{M}} \alpha_{0}
$$

Lemma 30. Suppose $G$ is acceptable. Then the same holds for $M$ and in fact

$$
\xi_{\rho_{M}}(h)=\xi_{\rho}\left(h_{1}\right) \quad(h \in A \cap M),
$$

where $h=h_{1} h_{2}\left(h_{1} \in A_{K}, h_{2} \in A_{\mathfrak{p}}\right)$ in the notation of $\S 16$.

Let $P_{+}$be the complement of $P_{M}$ in $P$. Then it is easy to verify that if $\alpha \in P_{+}$, the same holds for $-\theta \alpha$. This shows that $\rho-\rho_{M}=0$ on $\mathfrak{h} \cap \mathfrak{f}$. Let $\mathfrak{m}_{1}$ be the set of all $X \in \mathfrak{m}$ such that $\operatorname{tr}(\operatorname{ad} H \operatorname{ad} X)=0$ for $H \in \mathfrak{h} \cap \mathfrak{p}$. Then $\theta\left(\mathfrak{m}_{1}\right)=\mathfrak{m}_{1}$ and $(\mathfrak{h} \cap \mathfrak{p}) \cap \mathfrak{m}_{1}=\{0\}$. Hence if $M_{1}$ is the analytic subgroup of $G$ corresponding to $\mathrm{m}_{1}$, it is clear that $M=A_{\mathfrak{p}} M_{1}$ and $A_{\mathfrak{p}} \cap M_{1}=\{1\}$. Now $\mathrm{m}$ is reductive (Corollary 3 of Lemma 26) and $\mathfrak{h} \cap \mathfrak{p}$ lies in the center of $\mathfrak{m}$. Therefore since $\rho=\rho_{M}$ on $\mathfrak{h} \cap \mathfrak{f}$ and $A_{\mathfrak{p}}$ is simply connected, the statement of the lemma follows immediately by considering an acceptable complexification of $G$.

19. Behavior of $F$ around singular points. From now on we assume that $G$ is acceptable. Put

$$
\Delta_{A}(h)=\xi_{\rho}(h) \prod_{\alpha \in P}\left(1-\xi_{\alpha}(h)^{-1}\right) \quad(h \in A) .
$$

(We shall often drop the subscript $A$ if there is no risk of confusion.) Then $A^{\prime}=A \cap G^{\prime}$ is the set of all points $h \in A$, where $\Delta(h) \neq 0$. Put

$$
\Delta_{R}^{\prime}(h)=\prod_{\alpha \in P_{R}}\left(1-\xi_{\alpha}(h)^{-1}\right) \quad(h \in A),
$$

where $P_{R}$ is the set of all real roots (see [4(m), §4]) in $P$. Let $A^{\prime}(R)$ be the set of those $h \in A$ where $\Delta_{R}{ }^{\prime}(h) \neq 0$. We now use the notation of $\S 15$.

Lemma 31(13). Put $\Phi_{A}(h)=\Delta_{A}(h) F(h)\left(h \in A^{\prime} \cap \Omega\right)$. Then $\Phi_{A}$ can be extended to an analytic function on $A^{\prime}(R) \cap \Omega$.

(13) Cf. [4(n), Theorem 2] and [4(e), Theorem 8]. 
Fix a point $a \in A \cap \Omega$. Then $a$ is semisimple. We now use the notation of $\S 4$ and define $\Omega_{\Xi}=a^{-1} \Omega \cap \Xi^{\prime}$. Put $\sigma=\left|v_{a}\right|^{1 / 2} \sigma_{T}$ as in $\S 15$, and let $\Omega_{\Xi}{ }^{\prime}$ be the set of all elements in $\Omega_{\Xi}$ which are regular in $\Xi$. We denote by $g$, as before, the analytic function on $\Omega_{\Xi}{ }^{\prime}$ such that $g$ is locally summable on $\Omega_{\Xi}$ and $\sigma=g$ on $\Omega_{\Xi}$. Since $T$ is invariant, the same holds for $F$ and, as we have seen during the proof of Theorem 2,

$$
F(a y)=\left|v_{a}(y)\right|^{-1 / 2} g(y) \quad\left(y \in \Omega_{\Xi}{ }^{\prime}\right) .
$$

Now $\Omega_{\Xi}$ is an open and completely invariant neighborhood of 1 in $\Xi$. Hence (see $\$ 9$ ) we can choose an open and completely invariant neighborhood $U$ of zero in $z$ such that the exponential mapping defines an analytic diffeomorphism of $U$ onto an open subset $U_{\Xi}$ of $\Omega_{\Xi}$. Consider the function $\xi_{z}$ on $z$ (see $\S 10$ ) and, for any $\phi \in C_{c}{ }^{\infty}(U)$, define $f_{\phi} \in C_{c}^{\infty}\left(U_{\Xi}\right)$ by

$$
f_{\phi}(\exp Z)=\xi_{3}(Z)^{-1} \phi(Z) \quad(Z \in U) .
$$

Let $\tau$ be the distribution on $U$ given by $\tau(\phi)=\sigma\left(f_{\phi}\right)\left(\phi \in C_{c}^{\infty}(U)\right)$. Define $\mathfrak{B}=3(\mathfrak{3}) \cdot \mu(\mathfrak{U})$, where $\mu=\mu_{\mathfrak{g} / \mathfrak{3}}$ (in the notation of $\S 12$ ). Then we know from Lemmas 21 and 22 that $3(\mathfrak{3})$ is a finite module over $\mu(3)$ and $v \sigma=0(v \in \mathfrak{B})$. Let $z \rightarrow p_{z}$ denote the canonical isomorphism of $3(3)$ onto $I\left(3_{c}\right)$ (see $\left.\$ 12\right)$. Then $\partial\left(p_{v}\right) \tau=0(v \in \mathfrak{B})$ from the corollary of Lemma 24. Hence Theorem 1 of [4(n)] is applicable to $(z, U, \tau)$. Let $z^{\prime}$ denote the set of all elements of $z$ which are regular in $z$ and let $\psi$ be the analytic function on $U^{\prime}=U \cap z^{\prime}$ such that $\tau=\psi$.

LEMMA 32. $\psi(Z)=\xi_{3}(Z)\left|v_{a}(\exp Z)\right|^{1 / 2} F(a \exp Z)\left(Z \in U^{\prime}\right)$.

Fix $\phi \in C_{c}^{\infty}(U)$. Then

$$
\begin{aligned}
\int \phi \psi d Z & =\tau(\phi)=\sigma\left(f_{\phi}\right)=\int f_{\phi} g d y \\
& =\int \phi(Z) \xi_{3}(Z) g(\exp Z) d Z .
\end{aligned}
$$

Here $d y$ is the Haar measure of $\Xi$ and $d Z$ the Euclidean measure on $z$ and they are related (see $\$ 10)$ by the equation

$$
d y=\xi_{3}(Z)^{2} d Z \quad(y=\exp Z, Z \in U) .
$$

Since $\exp \left(U^{\prime}\right) \subset \Omega_{\Xi}{ }^{\prime}$, we have

$$
g(\exp Z)=\left|v_{a}(\exp Z)\right|^{1 / 2} F(a \exp Z) \quad\left(Z \in U^{\prime}\right)
$$

and so our assertion is now obvious.

Let $P_{3}$ be the set of all roots $\alpha \in P$ such that $\xi_{\alpha}(a)=1$. Put $P_{3, R}=P_{3} \cap P_{R}$ and let $\mathfrak{h}^{\prime}(R)$ be the set of all points $H \in \mathfrak{h}$, where $\prod_{\alpha \in P_{3, R}} \alpha(H) \neq 0$. Then we know from [4(n), Theorem 2] that there exists an analytic function $u$ on $\mathfrak{h}^{\prime}(R) \cap U$ such that 


$$
u(H)=\pi_{\mathfrak{z}}(H) \psi(H) \quad\left(H \in \mathfrak{h} \cap U^{\prime}\right),
$$

where $\pi_{3}=\prod_{\alpha \in P_{3}} \alpha$.

LEMMA 33. Let $\mathfrak{h}_{0}$ be an open and connected neighborhood of zero in $U \cap \mathfrak{h}$. Then

$$
\pi_{\mathfrak{3}}(H) \xi_{\mathfrak{z}}(H)\left|v_{a}(\exp H)\right|^{1 / 2}=c \Delta(a \exp H) \quad\left(H \in \mathfrak{h}_{0}\right),
$$

where $c$ is a constant. Let $P^{\prime}$ be the complement of $P_{3}$ in $P$ and $p$ the number of roots in $P^{\prime}$. Then $p=(\operatorname{dim} g-\operatorname{dim} \jmath) / 2$ and

$$
c^{2}=(-1)^{p} \operatorname{sign} v_{a}(1) .
$$

Finally

$$
c=\left|v_{a}(1)\right|^{1 / 2}\left\{\xi_{\rho}(a) \prod_{\alpha \in P^{\prime}}\left(1-\xi_{\alpha}(a)^{-1}\right)\right\}^{-1} .
$$

Put $\rho^{\prime}=(1 / 2) \sum_{\alpha \in P^{\prime}} \alpha$. Then it is clear that

$$
\begin{aligned}
v_{a}(\exp H) & =\prod_{\alpha \in P^{\prime}}\left\{\left(\xi_{\alpha}(a \exp H)^{-1}-1\right)\left(\xi_{\alpha}(a \exp H)-1\right)\right\} \\
& =(-1)^{p} \xi_{2 \rho^{\prime}}(a)\left\{e^{\rho^{\prime}(H)} \prod_{\alpha \in P^{\prime}}\left(1-\xi_{\alpha}(a \exp H)^{-1}\right)\right\}^{2}
\end{aligned}
$$

for $H \in \mathfrak{h}$. Since $v_{a}(\exp H)$ is real and $\neq 0$ for $H \in \mathfrak{h}_{0}$, it is clear that

$$
\left|v_{a}(\exp H)\right|^{-1 / 2} e^{\rho^{\prime}(H)} \prod_{\alpha \in P^{\prime}}\left(1-\xi_{\alpha}(a \exp H)^{-1}\right)
$$

is an analytic function on $\mathfrak{h}_{0}$ whose fourth power is a constant. Therefore since $\mathfrak{h}_{0}$ is connected, we conclude that

$$
\left|v_{a}(\exp H)\right|^{1 / 2}=c_{1} e^{\rho^{\prime}(H)} \prod_{\alpha \in P^{\prime}}\left(1-\xi_{\alpha}(a \exp H)^{-1}\right) \quad\left(H \in \mathfrak{h}_{0}\right),
$$

where

$$
c_{1}=\left|v_{a}(1)\right|^{1 / 2} \prod_{\alpha \in P^{\prime}}\left(1-\xi_{\alpha}(a)^{-1}\right)^{-1}
$$

A similar argument shows that

Hence

$$
\pi_{\mathfrak{z}}(H) \xi_{3}(H)=\prod_{\alpha \in P_{3}}\left(e^{\alpha(H) / 2}-e^{-\alpha(H) / 2}\right) \quad\left(H \in \mathfrak{h}_{0}\right)
$$

$$
\pi_{\mathfrak{3}}(H) \xi_{3}(H)\left|v_{a}(\exp H)\right|^{1 / 2}=c \Delta(a \exp H) \quad\left(H \in \mathfrak{h}_{0}\right),
$$

where $c=c_{1} \xi_{\rho}(a)^{-1}$.

It is obvious that $\operatorname{dim} g-\operatorname{dim} \mathfrak{z}=2 p$. Since $\xi_{\alpha}(a)=1$ for $\alpha \in P_{3}$, it is clear that $\xi_{2 \rho},(a)=\xi_{2 \rho}(a)$. Now

$$
\begin{aligned}
c_{1}^{2} \prod_{\alpha \in P^{\prime}}\left(1-\xi_{\alpha}\left(a^{-1}\right)\right)^{2} & =\left|v_{a}(1)\right|=v_{a}(1) \operatorname{sign} v_{a}(1) \\
& =(-1)^{p}\left(\operatorname{sign} v_{a}(1)\right) \xi_{2 \rho^{\prime}}(a) \prod_{\alpha \in P^{\prime}}\left(1-\xi_{\alpha}\left(a^{-1}\right)\right)^{2} .
\end{aligned}
$$


This shows that $c^{2}=(-1)^{p} \operatorname{sign} v_{a}(1)$.

It follows from Lemmas 32 and 33 that

$$
u(H)=c \Phi_{A}(a \exp H) \quad\left(H \in \mathfrak{h}_{0} \cap U^{\prime}\right) .
$$

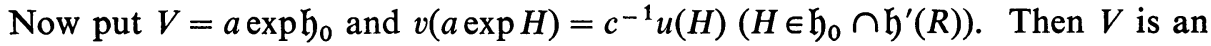
open neighborhood of $a$ in $A \cap \Omega, v$ is an analytic function on $V \cap A^{\prime}(R)$ and $v=\Phi_{A}$ on $V$. This proves Lemma 31 .

For any root $\alpha$, let $s_{\alpha}$ denote the Weyl reflexion corresponding to $\alpha$. The Weyl group $W$ of $(\mathfrak{g}, \mathfrak{h})$ operates on $\mathfrak{h}_{c}$ and therefore also on $\mathfrak{S}\left(\mathfrak{h}_{c}\right)$.

LEMMA 34. Fix a point $a \in A \cap \Omega$ and suppose $v$ is an element in $\mathfrak{S}\left(\mathfrak{h}_{c}\right)$ such that $v^{s_{\alpha}}=-v$ for every real root $\alpha$ for which $\xi_{\alpha}(a)=1$. Then $v \Phi_{A}$ can be extended to a continuous function around $a$.

We keep to the above notation. Then by Lemma 19 of $[4(\mathrm{n})] \partial(v) u \operatorname{can}\left({ }^{10}\right)$ be extended to a continuous function around zero. Since $\Phi_{A}(a \exp H)=c^{-1} u(H)$ $\left(H \in \mathfrak{h}_{0} \cap U^{\prime}\right)$, our assertion is now obvious.

For any root $\alpha$, define $H_{\alpha}$ as in [4(m), §4] and put $\varpi=\prod_{\alpha \in P} H_{\alpha} \in \subseteq\left(\mathfrak{h}_{c}\right)$. Then, by Lemma $34, \varpi \Phi_{A}$ can be extended to a continuous function $\Psi_{A}$ on $A$.

Lemma 35. Let $A$ and $B$ be two Cartan subgroups of $G$. Then $\Psi_{A}=\Psi_{B}$ on $A \cap B \cap \Omega$.

Fix $a \in A \cap B \cap \Omega$ and let $\mathfrak{a}$ and $\mathfrak{b}$ be the Cartan subalgebras corresponding to $\mathfrak{a}$ and $\mathfrak{b}$, respectively. Define $\mathfrak{z}, U$ and $\psi$ as in Lemma 32 . Then $\mathfrak{a}, \mathfrak{b}$ are Cartan subalgebras of $\mathfrak{z}$. Put $\mathfrak{h}=\mathfrak{a}$ or $\mathfrak{b}$ and define (14)

$$
\varpi_{\mathfrak{z}}^{\mathfrak{h}}=\varpi_{\mathfrak{z}}=\prod_{\alpha \in P_{\mathfrak{z}}} H_{\alpha}, \quad \varpi_{\mathfrak{g} / \mathfrak{3}}^{\mathfrak{h}}=\varpi_{\mathfrak{g} / \mathfrak{3}}=\prod_{\alpha \in P^{\prime}} H_{\alpha}
$$

in the notation introduced above. Then $\varpi=\varpi_{\mathfrak{z}} \cdot \varpi_{\mathfrak{g} / \mathbf{z}}$. Since $\varpi^{s_{\alpha}}=-\varpi, \varpi_{\mathbf{z}}^{s_{\alpha}}=-\varpi_{\mathbf{z}}$ for any $\alpha \in P_{\mathfrak{z}}$, it is clear that $\varpi_{\mathfrak{g} / \mathfrak{3}}$ is invariant under the Weyl group of $(\mathfrak{z}, \mathfrak{h})$ ). Therefore, by Chevalley's theorem [4(f), Lemma 9], there exists an element $\eta \in I\left(\mathfrak{z}_{c}\right)$ such that the projection $\eta_{\mathfrak{a}}$ of $\eta$ in $\mathfrak{S}\left(\mathfrak{a}_{c}\right)=S\left(\mathfrak{a}_{c}\right)$ (see $\left.[4(1), \S 8]\right)$ is $\varpi_{\mathfrak{g} / \mathfrak{a}}{ }^{a}$.

Let $G_{c}$ be an acceptable complexification of $G$ and $\Xi_{c}$ the analytic subgroup of $G_{c}$ corresponding to $\mathfrak{z}_{c}$. Then we can choose $y \in \Xi_{c}$ such that $\left(\mathfrak{a}_{c}\right)^{y}=\mathfrak{b}_{c}$. Thus we have an isomorphism $D \rightarrow D^{y}$ of $\mathfrak{D}\left(\mathfrak{a}_{c}\right)$ onto $\mathfrak{D}\left(\mathfrak{b}_{c}\right)$ (see [4(1), §3]). Since the definition of $\Psi_{B}$ is obviously independent of the order of roots, we may assume that the positive roots of $\mathfrak{a}$ are mapped into positive roots of $\mathfrak{b}$ under this isomorphism. Define $j$ as in $\$ 18$. Then it is obvious that $y j(a) y^{-1}=j(a)$. Therefore it follows from Lemma 33 that $c_{A}=c_{B}$ and $\eta_{\mathfrak{b}}=\varpi_{\mathfrak{g} / \mathbf{b}}{ }^{\mathfrak{b}}$. (Here $c_{A}$ and $c_{B}$ are the constants which correspond to $c$ of Lemma 33 for the cases $\mathfrak{h}=\mathfrak{a}$ and $\mathfrak{h}=\mathfrak{b}$, respectively.)

(14) We use a similar notation in other cases. For example $\pi_{\mathfrak{z}}^{\mathfrak{h}}=\pi_{\mathfrak{z}}$ and $\varpi^{\mathfrak{h}}=\varpi$. 
Now put

$$
u^{\mathfrak{h}}(H)=\pi_{\mathfrak{z}}^{\mathfrak{h}}(H) \psi(H) \quad\left(H \in U^{\prime} \cap \mathfrak{h}\right) .
$$

Then it follows from the corollary of Theorem 3 of $[4(n)]$ and $[4(f)$, Theorem 1$]$ (both applied to $z$ ) that

$$
\partial\left(\varpi_{\mathfrak{3}}^{\mathfrak{a}} \cdot \eta_{\mathfrak{a}}\right) u^{\mathfrak{a}}=\partial\left(\varpi_{\mathfrak{z}}^{\mathfrak{b}} \cdot \eta_{\mathfrak{b}}\right) u^{\mathfrak{b}}
$$

on $\mathfrak{a} \cap \mathfrak{b} \cap U$. This proves that $\partial\left(\varpi^{\mathfrak{a}}\right) u^{\mathfrak{a}}=\partial\left(\varpi^{\mathfrak{b}}\right) u^{\mathfrak{b}}$ on $\mathfrak{a} \cap \mathfrak{b} \cap \Omega$. But if $U_{0}$ is an open convex neighborhood of zero in $U$, we know that

$$
\begin{array}{ll}
u^{\mathfrak{a}}(H)=c_{A} \Phi_{A}(a \exp H) & \left(H \in U_{0}^{\prime} \cap \mathfrak{a}\right), \\
u^{\mathfrak{b}}(H)=c_{B} \Phi_{B}(a \exp H) & \left(H \in U_{0}^{\prime} \cap \mathfrak{b}\right),
\end{array}
$$

where $U_{0}^{\prime}=U_{0} \cap U^{\prime}$. Therefore since $c_{A}=c_{B} \neq 0$, we conclude that $\Psi_{A}(a)=\Psi_{B}(a)$.

20. The function $\nabla_{G} F$. We write $\varpi=\varpi_{A}$ for a given Cartan subgroup $A$.

LEMMA 36. There exists a unique differential operator $\nabla_{G}$ on $G^{\prime}$ with the following properties.

(1) $\nabla_{G}$ is invariant under $G$.

(2) Let $A$ be a Cartan subgroup of G. Then

$$
f\left(h ; \nabla_{G}\right)=f\left(h ; \varpi_{A} \circ \Delta_{A}\right)
$$

for $f \in C^{\infty}(G)$ and $h \in A \cap G^{\prime}$.

Moreover, $\nabla_{G}$ is analytic.

The proof is similar to that of [4(n), Lemma 24]. Since two distinct Cartan subgroups cannot have a regular element in common, the uniqueness is obvious. The existence is proved as follows. Fix a Cartan subgroup $A$ of $G$ and define $G_{A}=\bigcup_{x \in G} x A^{\prime} x^{-1}$, where $A^{\prime}=A \cap G^{\prime}$. Let $\mathfrak{h}$ be Cartan subalgebra of $A, \tilde{A}$ the normalizer of $\mathfrak{h}$ in $G$ and $\tilde{A}_{K}=\tilde{A} \cap K$ in the notation of $\$ 16$. Then by Corollary 2 of Lemma $26, \tilde{A}=\tilde{A}_{K} A_{\mathfrak{p}}$ and if $A_{0}$ is the center of $A$, it follows (see $\$ 16$ ) that

$$
W_{A}=\tilde{A} / A_{0} \simeq \tilde{A}_{K} / A_{0} \cap K
$$

is both compact and discrete and therefore it is finite. Let $x \rightarrow x^{*}$ denote the natural projection of $G$ on $G^{*}=G / A_{0}$. Define $h^{x^{*}}=h^{x}(h \in A, x \in G)$. Then the mapping $\phi:\left(x^{*}, h\right) \rightarrow h^{x^{*}}$ of $G^{*} \times A$ into $G$ is everywhere regular on $G^{*} \times A^{\prime}$. Hence $G_{A}=\phi\left(G^{*} \times A^{\prime}\right)$ is open in $G$. Now $W_{A}$ operates on $G^{*}$ and $A$ as follows. Let $y$ be an element in $A$ whose image in $W_{A}$ is $s$. Then

Define

$$
x^{*} s=(x y)^{*}(x \in G), h^{s}=y h y^{-1} .
$$

$$
\left(x^{*}, h\right) s=\left(x^{*} s, h^{s^{-1}}\right) \quad\left(x^{*} \in G^{*}, h \in A^{\prime}\right) .
$$


In this way $W_{A}$ operates on the right on $G^{*} \times A^{\prime}$ without fixed points and the quotient space $\left(G^{*} \times A^{\prime}\right) / W_{A}$ may be identified with $G_{A}$ by means of $\phi$. By making use of the homomorphism $j: G \rightarrow G_{c}$ (see $\S 18$ ) one proves without difficulty that the differential operator $\varpi_{A} \circ \Delta_{A}$ on $A$ is invariant under $W_{A}$. The rest of the proof now goes through exactly as in [4(n), §9].

LEMMA 37. For any $z \in 3,\left(\nabla_{G} \circ z\right) F$ can be extended to a continuous function on $\Omega$.

Since the distribution $z T$ also satisfies all the conditions of Theorem 2, it is enough to consider the case $z=1$. Let $\Omega_{0}$ be the set of all points $x_{0} \in \Omega_{0}$ for which there exists an open neighborhood $V$ of $x_{0}$ in $\Omega$ and a continuous function $v$ on $V$ such that $v=\nabla_{G} F$ on $V \cap G^{\prime}$. Obviously $\Omega_{0}$ is an open and invariant subset of $\Omega$. Hence, in view of Lemma 7 , it would be enough to prove that every semisimple element of $\Omega$ is contained in $\Omega_{0}$.

Fix a semisimple element $a \in \Omega$ and let us use the notation of Lemma 32 . Let $z^{\prime}$ be the set of those elements of $z$ which are regular in $\mathfrak{z}$. Define the differential operator $\nabla_{3}$ on $\mathfrak{z}^{\prime}$ as in $[4(\mathrm{n}), \S 9]$ and fix an open and convex neighborhood $U_{0}$ of zero in $U$ and put $U_{0}^{\prime}=U_{0} \cap U^{\prime}$. Let $a$ be a Cartan subalgebra of $z$. Then, as we have seen in $\S 19$, there exists a unique element $\eta \in I\left(z_{c}\right)$ such that $\eta_{\mathfrak{a}}=\varpi_{\mathfrak{g} / \mathfrak{z}}^{\mathfrak{a}}$. Let $c$ denote the constant of Lemma 33 corresponding to $\mathfrak{h}=\mathfrak{a}$.

LEMMA 38. $F\left(a \exp Z ; \nabla_{G}\right)=c \psi\left(Z ; \nabla_{3} \circ \partial(\eta)\right)\left(Z \in U_{0}^{\prime}\right)$.

Fix $H_{0} \in U_{0}^{\prime}$ and let $\mathfrak{h}$ be the centralizer of $H_{0}$ in $\mathfrak{z}$. Then $\mathfrak{h}$ is a Cartan subalgebra of $z$ and therefore also of $g$. Moreover, $a \exp H_{0} \in \Omega \cap G^{\prime}$. Let $A$ be the Cartan subgroup of $G$ corresponding to $\mathfrak{h}$. Then

$$
F\left(a \exp H_{0} ; \nabla_{G}\right)=F\left(a \exp H_{0} ; \varpi_{A} \circ \Delta_{A}\right),
$$

from the definition of $\nabla_{\mathfrak{G}}$. Put $\mathfrak{h}_{0}=\mathfrak{h} \cap U_{0}$. Then we have seen in $\S 19$ that

$$
\Delta_{A}(a \exp H) F(a \exp H)=c_{A} \pi_{\mathfrak{z}}(H) \psi(H) \quad\left(H \in \mathfrak{h}_{0} \cap U^{\prime}\right),
$$

where $c_{A}$ is a constant. Moreover, by a suitable choice of positive roots of $(\mathfrak{g}, \mathfrak{h})$ we can arrange (see the proof of Lemma 35) that $c_{A}=c$ and $\eta_{\mathfrak{h}}=\varpi_{\mathfrak{g} / \mathbf{3}}$. Then it follows from [4(f), Theorem 1] and the definition of $\nabla_{3}$ that

$$
\psi\left(H ; \nabla_{\mathfrak{3}} \circ \partial(\eta)\right)=\psi\left(H ; \partial(\varpi) \circ \pi_{\mathfrak{3}}\right) \quad\left(H \in \mathfrak{h}_{0} \cap U^{\prime}\right),
$$

where $\boldsymbol{m}=\boldsymbol{\varpi}_{\boldsymbol{A}}$. Therefore it is clear that

$$
F\left(a \exp H_{0} ; \nabla_{G}\right)=c \psi\left(H_{0} ; \nabla_{\mathbf{3}} \circ \partial(\eta)\right)
$$

and this proves our assertion.

It follows from Lemma 38 and [4(n), Lemma 25] that there exists an open neighborhood $V_{\Xi}$ of 1 in $\Omega_{\Xi}$ and a continuous function $g_{0}$ on $V_{\Xi}$ such that

$$
F\left(a y ; \nabla_{G}\right)=g_{0}(y) \quad\left(y \in V_{\Xi}^{\prime}=V_{\Xi} \cap \Omega_{\Xi}{ }^{\prime}\right)
$$


in the notation of $\S 19$. Let $x \rightarrow \bar{x}$ denote the natural mapping of $G$ on $\bar{G}=G / \Xi$. Select open neighborhoods $\bar{G}_{0}$ and $V_{0}$ of $\overline{1}$ and 1 in $\bar{G}$ and $V_{\Xi}$, respectively. If they are sufficiently small the following conditions hold. There exists an analytic mapping $\phi$ of $\bar{G}_{0}$ into $G$ such that: (1) $\overline{\phi(\bar{x})}=\bar{x}$ for $\bar{x} \in \bar{G}_{0}$ and (2) the mapping $\alpha:(\bar{x}, y) \rightarrow(a y)^{\phi(\bar{x})}$ of $\bar{G}_{0} \times V_{0}$ into $G$ is univalent and regular and $V=\alpha\left(\bar{G}_{0} \times V_{0}\right) \subset V_{\Xi}$. Then $V$ is an open neighborhood of $a$ in $G$ and $\alpha$ defines an analytic diffeomorphism of $\bar{G}_{0} \times V_{0}$ on $V$. Define a function $F_{0}$ on $V$ by

$$
F_{0}(\alpha(\bar{x}, y))=g_{0}(y) \quad\left(\bar{x} \in \bar{G}_{0}, y \in V_{0}\right) .
$$

Then $F_{0}$ is continuous and since $\nabla_{G} F$ is invariant under $G$, it is obvious that $F_{0}=\nabla_{G} F$ on $V \cap G^{\prime}$. This shows that $a \in \Omega_{0}$ and therefore Lemma 37 is proved.

21. An elementary result. Let $\mathfrak{h}$ be a Cartan subalgebra of $\mathfrak{g}$ and $W$ the Weyl group of $(\mathfrak{g}, \mathfrak{h})$.

Lemma 39. Let $\lambda$ be a linear function on $\mathfrak{h}_{c}$. Then there exists an invariant analytic function $f_{\lambda}$ on $\mathrm{g}$ such that

Moreover, $f_{\lambda}$ is unique.

$$
\pi(H) f_{\lambda}(H)=\sum_{s \in W} \varepsilon(s) e^{\lambda(s H)} \quad(H \in \mathfrak{h}) .
$$

Let $\mathfrak{h}^{\prime}$ be the set of all elements $H \in \mathfrak{h}$, where $\pi(H) \neq 0$. Since $\left(\mathfrak{h}^{\prime}\right)^{G}$ is an open subset of $\mathrm{g}$, the uniqueness of $f_{\lambda}$ is obvious. Therefore it remains to prove its existence. For this we may obviously assume that $g$ is semisimple and $G$ is the connected adjoint group of $\mathrm{g}$. Now we use the notation of $\S 16$. Let $G_{c}$ be the (connected) complex adjoint group of $\mathfrak{g}_{c}$ and $U$ the real analytic subgroup of $G_{c}$ corresponding to the compact real form $\mathfrak{u}=\mathfrak{f}+(-1)^{1 / 2} \mathfrak{p}$ of $\mathfrak{g}_{c}$. Then $U$ is compact. Put $B(X, Y)=\operatorname{tr}(\operatorname{ad} X$ ad $Y)\left(X, Y \in \mathfrak{g}_{c}\right)$ as usual and consider

$$
f(X: Y)=\int_{U} \exp B(u X, Y) d u \quad\left(X, Y \in \mathfrak{g}_{c}\right),
$$

where $d u$ is the normalized Haar measure on $U$. Then $f$ is obviously a holomorphic function on $\mathfrak{g}_{c} \times \mathfrak{g}_{c}$ and it is clear that

$$
f(X ; \tau(Z): Y)=0 \quad(Z \in \mathfrak{u})
$$

in the notation of [4(1), \&4]. Since $f$ is holomorphic in $X$, this implies that $f(x X: Y)=f(X: Y)$ for $x \in G_{c}$.

Let $H_{\lambda}$ denote the element in $\mathfrak{h}_{c}$ such that $B\left(H, H_{\lambda}\right)=\lambda(H)$ for all $H \in \mathfrak{h}_{c}$. Then we know from [4(f), Theorem 2] that

$$
\pi\left(H_{\lambda}\right) \pi(H) f\left(H: H_{\lambda}\right)=c \sum_{s \in W} \varepsilon(s) e^{\lambda(s H)} \quad\left(H \in \mathfrak{h}_{c}\right),
$$

where $c$ is a number $\neq 0$ independent of $H$ and $\lambda$. Therefore we can take

$$
f_{\lambda}(X)=c^{-1} \pi\left(H_{\lambda}\right) f\left(X: H_{\lambda}\right) \quad(X \in \mathfrak{g}) .
$$


22. The invariant integral on $G$. We now return to the notation of $\S 19$. Let $A_{0}$ denote the center of $A$ and $x \rightarrow x^{*}$ the natural projection of $G$ on $G^{*}=G / A_{0}$. Put $h^{x^{*}}=h^{x}(h \in A, x \in G)$ and let $d x^{*}$ denote the invariant measure on $G^{*}$. For any $f \in C_{c}{ }^{\infty}(G)$, put

$$
F_{f}(h)=\varepsilon_{R}(h) \Delta(h) \int_{G^{*}} f\left(h^{x^{*}}\right) d x^{*} \quad\left(h \in A^{\prime}\right),
$$

where $A^{\prime}=A \cap G^{\prime}$ and $\varepsilon_{R}(h)=\operatorname{sign} \Delta_{R}{ }^{\prime}(h)$. Then $F_{f}$ is a $C^{\infty}$-function on $A^{\prime}$ and if $\gamma$ is the canonical isomorphism of 3 onto $I\left(\mathfrak{h}_{c}\right)$ (see $\left.\S 6\right)$, we have [4(h), Theorem 3]

$$
F_{z f}=\gamma(z) F_{f} \quad\left(z \in \mathcal{B}, f \in C_{c}^{\infty}(G)\right) .
$$

Let $S_{I}$ denote the set of all positive singular imaginary roots of $(\mathfrak{g}, \mathfrak{h})$ (see $[4(\mathrm{~m}), \S 4])$. Define

$$
\Delta_{I}^{\prime}(h)=\prod_{\alpha \in S_{I}}\left(1-\xi_{\alpha}(h)^{-1}\right) \quad(h \in A)
$$

and let $A^{\prime}(I)$ be the set of those points $h \in A$ where $\Delta_{I}{ }^{\prime}(h) \neq 0$.

Lemma 40. Fix $f \in C_{c}{ }^{\infty}(G)$. Then $F_{f}$ can be extended to a $C^{\infty}$-function on $A^{\prime}(I)$. Let $a$ be a point in $A$ and $v$ an element in $\mathfrak{S}\left(\mathfrak{h}_{c}\right)$ such that $v^{s_{\alpha}}=-v$ for every singular imaginary root $\alpha$ for which $\xi_{\alpha}(a)=1$. Then $v F_{f}$ can be extended to a continuous function around $a$.

Let $z$ and $\Xi_{1}$ denote the centralizers of $a$ in $\mathfrak{g}$ and $G$, respectively, and $\Xi$ the connected component of 1 in $\Xi_{1}$. Then if $Z$ is the center of $G, \Xi_{1} / Z \Xi$ is finite (see [4( $\left.g_{2}\right)$, Lemma 15]). Choose an open neighborhood $B$ of 1 in $A$ with the following property (see [4(h), Theorem 1]). If $h \in B$ and $x \in G$ vary in such a way that $(a h)^{x}$ stays inside some compact subset of $G$, then the coset $\bar{x}=x \Xi_{1}$ remains within a compact subset of $\bar{G}=G / \Xi_{1}$. Let $x \rightarrow \bar{x}$ denote the natural projection of $G$ on $\bar{G}$. Since $z$ is reductive and $\Xi_{1} / Z \Xi$ is finite, it follows that the group $\Xi_{1}$ is unimodular. Hence we have an invariant measure $d \bar{x}$ on $\bar{G}$. Let $d y^{*}$ denote the invariant measure on $\Xi_{1}{ }^{*}=\Xi_{1} / A_{0}$. Then if $d \bar{x}$ and $d y^{*}$ are suitably normalized, we have

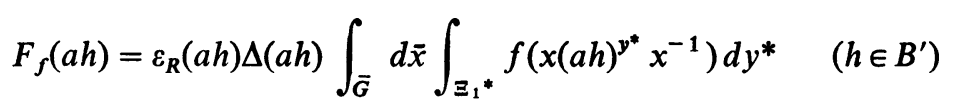

for $f \in C_{c}{ }^{\infty}(G)$. Here $B^{\prime}=B \cap a^{-1} A^{\prime}$. Now fix an open and relatively compact subset $G_{0}$ of $G$ and choose a compact set $\bar{\Omega}$ in $\bar{G}$ such that $(a B)^{x} \cap G_{0}=\varnothing(x \in G)$ unless $\bar{x} \in \bar{\Omega}$. Let $d y$ denote the Haar measure of $\Xi_{1}$ and choose $\gamma \in C_{c}{ }^{\infty}(G)$ such that

$$
\int_{\Xi Z} \gamma(x y) d y=1
$$

if $\bar{x} \in \bar{\Omega}(x \in G)$. Then if $d y$ is suitably normalized, we have

$$
F_{f}(a h)=\varepsilon_{R}(a h) \Delta(a h) \int_{G} \gamma(x) d x \int_{\Xi^{*}} f\left(x\left(a h^{y^{*}}\right) x^{-1}\right) d y^{*} \quad\left(h \in B^{\prime}\right)
$$


for $f \in C_{c}^{\infty}\left(G_{0}\right)$. Here $\Xi^{*}=\Xi / \Xi \cap A_{0}$. Now fix $f \in C_{c}{ }^{\infty}\left(G_{0}\right)$ and put

$$
g_{0}(y)=\int_{G} \gamma(x) f\left(x(a y) x^{-1}\right) d x \quad(y \in \Xi) .
$$

Then $g_{0} \in C_{c}{ }^{\infty}(\Xi)$.

We now use the notation of $\S 19$. Select an open and connected neighborhood $\mathfrak{h}_{0}$ of zero in $\mathfrak{h}$ such that $\operatorname{exph} \mathfrak{h}_{0} \subset B, \xi_{\alpha}(a \exp H) \neq 1\left(\alpha \in P^{\prime}\right)$ and

$$
\left(1-e^{-\alpha(H)}\right) / \alpha(H) \neq 0 \quad\left(\alpha \in P_{3}\right)
$$

for $H \in \mathfrak{h}_{0}$. Then if $\mathfrak{h}_{0}{ }^{\prime}$ is the set of all points $H \in \mathfrak{h}_{0}$, where $\pi_{\mathfrak{z}}(H) \neq 0$, it is clear

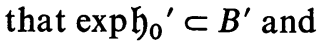

$$
F_{f}(a \exp H)=\varepsilon_{R}(a \exp H) \Delta(a \exp H) \int_{\Xi^{*}} g_{0}\left((\exp H)^{y^{*}}\right) d y^{*} \quad\left(H \in \mathfrak{h}_{0}{ }^{\prime}\right)
$$

A simple argument shows (see $\$ 21)$ that there exists an analytic function $D_{a}$ on 3 such that: (1) $D_{a}$ is invariant under $\Xi$ and (2) $\Delta(a \exp H)=\pi_{\mathfrak{z}}(H) D_{a}(H)$ for $H \in \mathfrak{h}$. Fix an open and completely invariant neighborhood $z_{0}$ of zero in $z$ such that the exponential mapping (from $z$ to $\Xi$ ) is regular and univalent on $z_{0}$ and select a $C^{\infty}$ function $u$ on $z$ such that: (1) $u$ is invariant under $\Xi$, (2) $\operatorname{Supp} u \subset z_{0}$, and (3) $u=1$ around zero. This is possible (see $\S 9$ and [4(n), Corollary 1 of Lemma 45]). Now put

$$
g(Z)=u(Z) D_{a}(Z) g_{0}(\exp Z) \quad(Z \in z) .
$$

Then $g \in C_{c}{ }^{\infty}\left(\mathfrak{z}_{0}\right)$. Since $\mathfrak{h}_{0}$ is connected and $\xi_{\alpha}(a \exp H) \neq 1$ for $\alpha \in P^{\prime}$ and $H \in \mathfrak{h}_{0}$, it is clear that

where

$$
\varepsilon_{R}(a \exp H)=\varepsilon_{3, R}(H) \varepsilon_{a} \quad\left(H \in \mathfrak{h}_{0}\right),
$$

$$
\varepsilon_{3, R}(H)=\operatorname{sign} \prod_{\alpha \in P_{z} \cap P_{R}} \alpha(H)
$$

and $\varepsilon_{a}$ is a constant. Therefore

$$
F_{f}(a \exp H)=\varepsilon_{a} \varepsilon_{3, R}(H) \pi_{3}(H) \int_{\Xi^{*}} g\left(y^{*} H\right) d y^{*} \quad\left(H \in U \cap \mathfrak{h}_{0}^{\prime}\right)
$$

where $U$ is an open neighborhood of zero in $z$ such that $u=1$ on $U$. The second assertion of the lemma now follows by applying Theorem 1 of $[4(m)]$ to $(\mathfrak{z}, \mathfrak{h})$ and $g$. Moreover, this obviously implies the first assertion.

Corollary. $\varpi F_{f}$ can be extended to a continuous function on $A$.

Since $\varpi^{s_{\alpha}}=-\varpi$ for every $\operatorname{root} \alpha$, this is an immediate consequence of Lemma 40 .

23. Statement of Theorem 3. Define $G_{A}$ as in $\$ 20$. Since $A_{0}$ is abelian and $A / A_{0}$ is finite (see the proof of Lemma 36), the Haar measure $d h$ of $A$ is biinvariant. We keep to the notation of $\$ 22$.

LEMMA 41. There exists a number $c>0$ such that 
for $f \in C_{c}\left(G_{A}\right)$.

$$
\int_{G} f(x) d x=c \int_{A}|\Delta(h)|^{2} d h \int_{G^{*}} f\left(h^{x^{*}}\right) d x^{*}
$$

We observe that

$$
\operatorname{det}\left(\operatorname{Ad}(h)^{-1}-1\right)_{\mathfrak{g} / \mathfrak{h}}=(-1)^{r} \Delta(h)^{2} \quad(h \in A),
$$

where $r$ is the number of positive roots of $(\mathfrak{g}, \mathfrak{h})$. From this our assertion follows in the usual way (see the proof of Lemma 36 and [4(c), p. 508]).

Corollary. Let $f \in C_{c}{ }^{\infty}(G)$. Then

$$
\int_{A}\left|\Delta(h) F_{f}(h)\right| d h \leqq c^{-1} \int_{G_{A}}|f(x)| d x .
$$

This is obvious from the above lemma.

We now use the notation of $\$ 16$. Let $\mathfrak{m}$ be the centralizer of $\mathfrak{h} \cap \mathfrak{p}$ in $\mathfrak{g}$ and $M$ the analytic subgroup of $G$ corresponding to $m$. Let $P_{M}$ be the set of all positive roots of $(\mathfrak{g}, \mathfrak{h})$ which vanish identically on $\mathfrak{h} \cap \mathfrak{p}$. Then $P_{M}$ is also the set of all positive imaginary roots of $(\mathfrak{g}, \mathfrak{h})$ or, equivalently, the set of positive roots of $(\mathfrak{m}, \mathfrak{h})$. Put

$$
\Delta_{M}(h)=\xi_{\rho}\left(h_{1}\right) \prod_{\alpha \in P_{M}}\left(1-\xi_{\alpha}\left(h^{-1}\right)\right) \quad(h \in A),
$$

where $h=h_{1} h_{2}\left(h_{1} \in A_{K}, h_{2} \in A_{\mathfrak{p}}\right)$. It follows from [4(h), Theorem 2] that

$$
\int_{A}\left|\Delta_{M}(h) F_{f}(h)\right| d h<\infty \quad\left(f \in C_{c}{ }^{\infty}(G)\right) .
$$

THEOREM 3. Let $v$ be a seminorm(15) on the complex vector space $C_{c}{ }^{\infty}(G)$ and $3_{0}$ a subalgebra of 3 containing 1 . Assume that 3 is a finite module over 30 and

$$
\int_{A}\left|\Delta_{M}(h) F_{f}(h)\right| d h \leqq v(f) \quad\left(f \in C_{c}{ }^{\infty}(G)\right) .
$$

Then for any $u \in \mathfrak{S}\left(\mathfrak{h}_{c}\right)$, we can choose a finite set of elements $z_{1}, \cdots, z_{N} \in \mathcal{Z}_{0}$ such that

$$
\sup _{h \in A^{\prime}}\left|F_{f}(h ; u)\right| \leqq \sum_{1 \leqq i \leqq N} v\left(z_{i} f\right) \quad\left(f \in C_{c}{ }^{\infty}(G)\right) .
$$

REMARK. The above form of this theorem suggested itself to me after a conversation with R.P. Langlands. My original version was less comprehensive.

Let $Z$ be the center of $G$ and $V$ a subset of $A$ such that $V Z=A$. Put $V^{\prime}=V \cap A^{\prime}$. We claim that it would be sufficient to prove the following lemma for a conveniently chosen $V$.

LEMMA 42. For any $u \in \mathfrak{S}\left(\mathfrak{h}_{c}\right)$, we can choose $z_{1}, \cdots, z_{N}$ in $\mathfrak{3}_{0}$ such that

(15) Here we ignore completely the topology of $C_{c}^{\infty}(G)$. 


$$
\sup _{h^{\prime} \in=V}\left|F_{f}(h ; u)\right| \leqq \sum_{1 \leqq i \leqq N} v\left(z_{i} f\right) \quad\left(f \in C_{c}^{\infty}(G)\right) .
$$

Put:

$$
v_{0}(f)=\int_{A}\left|\Delta_{M}(h) F_{f}(h)\right| d h \quad\left(f \in C_{c}{ }^{\infty}(G)\right) .
$$

Then $v_{0}$ is a seminorm on $C_{c}{ }^{\infty}(G)$ which satisfies the condition of Theorem 3. For any $y \in Z$, let $f_{y}$ denote the function $x \rightarrow f(x y)$ on $G$. Then it is clear that $\xi_{\rho}(y) F_{f_{y}}(h)=F_{f}(h y)\left(h \in A^{\prime}\right)$ and $\Delta_{M}(h y)=\xi_{\rho}(y) \Delta_{M}(h)$. Since $\left|\xi_{\rho}(y)\right|=1$, it follows that $v_{0}\left(f_{y}\right)=v_{0}(f)$. Therefore if Lemma 42 holds for $v_{0}$, we can conclude that

$$
\begin{aligned}
\sup _{h \in V^{\prime}}\left|F_{f}(h y ; u)\right| & =\sup _{h \in V^{\prime}}\left|F_{f_{y}}(h ; u)\right| \\
& \leqq \sum_{1 \leqq i \leqq N} v_{0}\left(z_{i} f_{y}\right)=\sum_{i} v_{0}\left(z_{i} f\right) \quad(y \in Z)
\end{aligned}
$$

since $z_{i} f_{y}=\left(z_{i} f\right)_{y}$. Now fix a seminorm $v$ as in Theorem 3. Then $v_{0}(g) \leqq v(g)$ for $g \in C_{c}{ }^{\infty}(G)$. Hence

$$
\sup _{h \in V}\left|F_{f}(h y ; u)\right| \leqq \sum_{i} v\left(z_{i} f\right) \quad\left(y \in Z, f \in C_{c}{ }^{\infty}(G)\right) .
$$

But since $V^{\prime} Z=A^{\prime}$, the assertion of Theorem 3 now follows immediately.

24. Reduction to $\mathfrak{h}$ in a special case. So now it remains to prove Lemma 42. First assume that every root of $(\mathfrak{g}, \mathfrak{h})$ is imaginary. Then $M=G$ and it follows from Corollary 5 of Lemma 26 that $A / Z$ is compact. So we can take $V$ to be compact. Let $\mathscr{S}$ be the set of all seminorms $\sigma$ on $\left({ }^{15}\right) C_{c}{ }^{\infty}(G)$ with the following property. We can choose a finite number of elements $z_{1}, \cdots, z_{N}$ in $3_{0}$ such that

$$
\sigma(f) \leqq \sum_{1 \leqq i \leqq N} v\left(z_{i} f\right) \quad\left(f \in C_{c}^{\infty}(G)\right) .
$$

Then since $V$ is compact, it would obviously be enough to prove the following result.

LEMMA 43. Given $h_{0} \in A$, we can choose an open neighborhood $U$ of $h_{0}$ in $A$ with the following property. For any $u \in \mathfrak{S}\left(\mathfrak{h}_{c}\right)$, there exists an element $\sigma_{u} \in \mathscr{S}$ such that

$$
\sup _{h \in A^{\prime} \cap U}\left|F_{f}(h ; u)\right| \leqq \sigma_{u}(f) \quad\left(f \in C_{c}^{\infty}(G)\right) .
$$

Let $c$ be the center and $g_{1}$ the derived algebra of $\mathfrak{g}$. Then $\mathfrak{h}=\mathfrak{c}+\mathfrak{h}_{1}$, where $\mathfrak{h}_{1}=\mathfrak{h} \cap \mathfrak{g}_{1}$. Since every root of $(\mathfrak{g}, \mathfrak{h})$ is imaginary, $-\operatorname{tr}(\operatorname{ad} H)^{2}\left(H \in \mathfrak{h}_{1}\right)$ is a positive-definite quadratic form on $\mathfrak{h}_{1}$. We extend it to a positive-definite quadratic form $Q$ on $\mathfrak{h}$ in such a way that $\mathfrak{c}$ and $\mathfrak{h}_{1}$ are orthogonal under $Q$ and, moreover, regard $\mathfrak{h}$ as a real Hilbert space under the norm $\|H\|^{2}=Q(H)(H \in \mathfrak{h})$.

Let us now introduce the notation of $\$ 19$ corresponding to $a=h_{0}$. Fix a number 
$c(0<c \leqq 1)$ and let $V$ be the set of all $H \in \mathfrak{h}$ with $\|H\|<c$. We assume that $c$ is so small that:

(1) $\left|\left(e^{\alpha(\boldsymbol{H}) / 2}-e^{-\alpha(H) / 2} \xi_{\alpha}\left(h_{0}^{-1}\right)\right)\right| \geqq(1 / 2)\left|1-\xi_{\alpha}\left(h_{0}^{-1}\right)\right|$ for every root $\alpha$ of $(\mathfrak{g}, \mathfrak{h})$ and $H \in V$.

(2) The exponential mapping of $V$ into $A$ is univalent.

(3) $\left|\left\{\left(e^{\alpha(H) / 2}-e^{-\alpha(H) / 2}\right) / \alpha(H)\right\}\right| \geqq 1 / 2$ for $\alpha \in P_{3}$ and $H \in V$.

Let $\mathfrak{h}^{\prime}$ be the set of all $H \in \mathfrak{h}$ where $\pi_{\mathfrak{z}}(H) \neq 0$. Then $V^{\prime}=V \cap \mathfrak{h}^{\prime}$ consists of a finite number of connected components, say $V_{1}, \cdots, V_{q}$. Put

$$
U=h_{0} \exp (a V),
$$

where $a$ is a positive number $(0<a \leqq 1)$. Then it is clear that

$$
U \cap A^{\prime}=\bigcup_{1 \leqq i \leqq q} U_{i}
$$

where $U_{i}=h_{0} \exp \left(a V_{i}\right)$. Then it would be sufficient to prove the following lemma.

LEMMA 44. Fix $i(1 \leqq i \leqq q)$. Then we can select a number $a(0<a \leqq 1)$ with the following property. For any $u \in \mathfrak{S}\left(\mathfrak{h}_{c}\right)$ we can choose $\sigma \in \mathscr{S}$ such that

$$
\sup _{\boldsymbol{B \in a V _ { l }}}\left|F_{f}\left(h_{0} \exp H ; u\right)\right| \leqq \sigma(f) \quad\left(f \in C_{c}{ }^{\infty}(G)\right)
$$

Put

$$
\phi_{f}(H)=F_{f}\left(h_{0} \exp H\right) \quad\left(f \in C_{c}{ }^{\infty}(G), H \in V^{\prime}\right) .
$$

Then it follows from [4(h), Theorem 3] that $\left({ }^{10}\right) \phi_{z f}=\partial(\gamma(z)) \phi_{f}$ for $z \in 3$. Moreover, it is obvious that Lemma 44 is equivalent to the following.

LEMMA 45. Fix $i(1 \leqq i \leqq q)$. Then we can select $a(0<a \leqq 1)$ with the following property. For any $u \in S\left(\mathfrak{h}_{c}\right)$, we can choose $\sigma \in \mathscr{S}$ such that

$$
\sup _{H \in a V_{i}}\left|\phi_{f}(H ; \partial(u))\right| \leqq \sigma(f)
$$

for all $f \in C_{c}^{\infty}(G)$.

We may assume that $i=1$. Let $L$ be the rank of $z_{1}=[3,3]$. Then we can choose $L$ roots $\alpha_{1}, \cdots, \alpha_{L}$ of $(\mathfrak{z}, \mathfrak{h})$ with the following property. If $\alpha$ is a root of $(\mathfrak{z}, \mathfrak{h})$ such that $(-1)^{1 / 2} \alpha(H)>0$ for $H \in V_{1}$, then $\alpha=\sum_{1 \leqq i \leqq L} m_{i} \alpha_{i}$, where $m_{i}$ are rational integers $\geqq 0$. Put $t_{i}(H)=(-1)^{1 / 2} \alpha_{i}(H)(1 \leqq i \leqq L, H \in \mathfrak{h})$ and choose a base $H_{j}\left(1 \leqq j \leqq l_{1}\right)$ for $\mathfrak{h}_{1}=\mathfrak{h} \cap \mathfrak{g}_{1}$ such that $t_{i}\left(H_{j}\right)=\delta_{i j}\left(1 \leqq i \leqq L, 1 \leqq j \leqq l_{1}\right)$. Let $H_{j}\left(l_{1}<j \leqq l\right)$ be an orthonormal base for $c$. Extend $\left(t_{1}, \cdots, t_{L}\right)$ to a Cartesian coordinate system $\left(t_{1}, \cdots, t_{l}\right)$ on $\mathfrak{h}$ by defining $t_{i}\left(H_{j}\right)=\delta_{i j}(1 \leqq i, j \leqq l)$. Then a point $H \in V$ lies in $V_{1}$ if and only if $t_{i}(H)>0(1 \leqq i \leqq L)$. Define

$$
\tau(H)= \begin{cases}\min \left|t_{i}(H)\right| & \text { if } L>0, \\ c & \text { if } L=0 \quad(H \in \mathfrak{h}) .\end{cases}
$$


Clearly $\|H\|^{2} \geqq 2|\alpha(H)|^{2}$ for any root $\alpha$ of $(\mathfrak{g}, \mathfrak{h})$. Hence if $H_{0} \in V_{1} / 2$ and $\left\|H-H_{0}\right\| \leqq \tau\left(H_{0}\right) / 2 \quad(H \in \mathfrak{h})$, it is clear that $\|H\| \leqq\left\|H_{0}\right\|+\tau\left(H_{0}\right) / 2<c$. Therefore $H \in V$. Moreover, $\left|t_{i}\left(H-H_{0}\right)\right|=\left|\alpha_{i}\left(H-H_{0}\right)\right| \leqq\left\|H-H_{0}\right\| \leqq \frac{1}{2} \tau\left(H_{0}\right) \leqq \frac{1}{2} t_{i}\left(H_{0}\right) \quad(1 \leqq i \leqq L)$. Therefore $t_{i}(H) \geqq \tau\left(H_{0}\right) / 2 \quad(1 \leqq i \leqq L)$ and so $H \in V_{1}$. This also shows that $\tau(H) \geqq \tau\left(H_{0}\right) / 2$. Thus we have obtained the following result.

LeMma 46. Fix $H_{0} \in V_{1} / 2$ and let $H$ be an element in $\mathfrak{h}$ such that

$$
\left\|H-H_{0}\right\| \leqq \tau\left(H_{0}\right) / 2 .
$$

Then $H \in V_{1}$ and $\tau(H) \geqq \tau\left(H_{0}\right) / 2$.

Fix a function $\psi$ on $\boldsymbol{R}$ of class $C^{\infty}$ such that $\psi=1$ on the interval $(-\infty, 0]$, $\psi=0$ on the interval $[1,+\infty)$ and $0 \leqq \psi \leqq 1$ everywhere.

LeMMA 47. For any real number $\varepsilon(0<\varepsilon \leqq 1 / 2)$ define

$$
\Psi_{\varepsilon}(H)=\psi\left(\varepsilon^{-1}\|H\|-2\right) \quad(H \in \mathfrak{h}) .
$$

Then for any element $u \in S\left(\mathfrak{h}_{c}\right)$ of degree $\leqq d$, we can choose a number $b>0$ such that

$$
\left|\Psi_{\varepsilon}(H ; \partial(u))\right| \leqq b \varepsilon^{-d}
$$

for all $H \in \mathfrak{h}$ and $0<\varepsilon \leqq 1 / 2$.

This is an immediate consequence of Lemma 55 of the Appendix (see also $\left[6\right.$, p. 281]). Observe that $\Psi_{\varepsilon}(H)=0$ unless $\|H\| \leqq 3 \varepsilon$.

25. Proof of a weaker result. Now first we prove the following weaker form (16) of Lemma 45.

Lemma 48. Given $u \in S\left(\mathfrak{h}_{c}\right)$, we can choose an integer $q \geqq 0$ and $\sigma \in \mathscr{S}$ such that

$$
\sup _{H \in V_{1} / 2}\left\{\prod_{1 \leqq i \leqq L} t_{i}(H)\right\}^{q}\left|\phi_{f}(H ; \partial(u))\right| \leqq \sigma(f)
$$

for all $f \in C_{c}^{\infty}(G)$.

Let $\omega_{1} \in 3$ be the Casimir operator (see [4(e), p. 140]) corresponding to $\mathrm{g}_{1}$. Put

$$
\omega=\omega_{1}-\sum_{l_{1}<j \leqq l} H_{j}^{2} \in 3 .
$$

Then it is easy to verify (see [4(e), p. 144]) that $\left({ }^{17}\right) \gamma(\omega)+\langle\rho, \rho\rangle$ is homo-

(16) Cf. [4(g), p. 206].

(17) $\langle\rho, \rho\rangle=\rho\left(H_{\rho}\right)$, where $H_{\rho}$ is the unique element in $\mathfrak{h}_{1 c}$ such that $\operatorname{tr}\left(\operatorname{ad} H \operatorname{ad} H_{\rho}\right)=\rho(H)$ for all $H \in \mathfrak{h}$. 
geneous of degree 2 and $D=\partial(\gamma(\omega))+\langle\rho, \rho>$ is an elliptic differential operator on $\mathfrak{h}$.

Now 3 being a finite module over $3_{0}$, we can choose $v_{1}=1, v_{2}, \cdots, v_{r}$ in 3 such that

$$
3=\sum_{1 \leqq i \leqq r} 3_{0} v_{i}
$$

Fix an integer $m \geqq 1$. Then we have an equation of the form

$$
\omega_{0}^{m r}+\sum_{1 \leqq j \leqq r} z_{j} \omega_{0}^{m(r-j)}=0,
$$

where $\omega_{0}=\omega+<\rho, \rho>$ and $z_{j} \in 3_{0}$.

For the proof of Lemma 48, we may obviously assume that $u \neq 0$. Let $d$ be the degree of $u$. Fix a Euclidean measure $d H$ on $\mathfrak{h}$ such that $d H$ corresponds, locally, to the Haar measure $d h$ on $A$, under the exponential mapping. Then if $m$ is sufficiently large, there exists a function $E_{0}$ on $\mathfrak{h}$ of class $C^{2 m(r-1)+d}$ such that

$$
D^{m r} E_{0}=\delta
$$

in the sense of the theory of distributions on the Euclidean space $\mathfrak{h}$ (with respect to the measure $d H$ ). Here $\delta$ is the Dirac measure on $\mathfrak{h}$ concentrated at zero and $E_{0}$ is of class $C^{\infty}$ everywhere except at the origin (see Lemma 57, §29). Put $E=\partial(u)^{*} E_{0}$, where the star denotes adjoint. It follows by applying the homomorphism $\gamma$ to the relation above that

$$
D^{m r}+\sum_{1 \leqq j \leqq r} \partial\left(\gamma\left(z_{j}\right)\right) D^{m(r-j)}=0 .
$$

Since $D^{*}=D$, we find, by taking adjoints, that

$$
D^{m r}+\sum_{1 \leqq j \leqq r} \partial\left(\gamma\left(z_{j}\right)\right)^{*} D^{m(r-j)}=0 .
$$

Put $E_{j}=-D^{m(r-j)} E(1 \leqq j \leqq r)$. Then $E_{j}$ is a function of class $C^{2 m(j-1)}$ and

$$
\partial(u)^{*} \delta=\sum_{1 \leqq j \leqq r} \partial\left(\gamma\left(z_{j}\right)\right)^{*} E_{j}
$$

Clearly $E_{j}$ is of class $C^{\infty}$ everywhere except at zero. Put $E_{j, \varepsilon}=\Psi_{\varepsilon} E_{j}$ for any $\varepsilon$ $(0<\varepsilon \leqq 1 / 3)$ in the notation of Lemma 47 . Then it is clear that

$$
\sum_{1 \leqq j \leqq r} \partial\left(\gamma\left(z_{j}\right)\right)^{*} E_{j, \varepsilon}=\partial(u)^{*} \delta+\beta_{\varepsilon},
$$

where $\beta_{\varepsilon} \in C_{c}^{\infty}(\mathfrak{h})$ and $\operatorname{Supp} \beta_{\varepsilon} \subset \operatorname{Supp} \Psi_{\varepsilon}$. Now $\Psi_{\varepsilon}(H)=1$ if $\|H\| \leqq 2 \varepsilon$. Hence $\beta_{\varepsilon}(H)=0$ unless $2 \varepsilon \leqq\|H\| \leqq 3 \varepsilon$.

Therefore

$$
\sup _{H}\left|\beta_{\varepsilon}(H)\right|=\sup _{2 \varepsilon \leqq\|B\| \leqq 3 \varepsilon}\left|\beta_{\varepsilon}(H)\right|
$$


Making use of Lemma 47 and the explicit formula for $E_{0}$ (see §29), we find that

$$
\sup _{H}\left|\beta_{\varepsilon}(H)\right| \leqq b_{1} \varepsilon^{-p+1}|\log \varepsilon| \leqq b_{2} \varepsilon^{-p}
$$

where $b_{1}, b_{2}$ are positive numbers and $p$ an integer $\geqq 0$, all independent of $\varepsilon(0<\varepsilon \leqq 1 / 3)$.

Now fix $H_{0} \in V_{1} / 2$ and put $\varepsilon_{0}=\tau\left(H_{0}\right) / 6, E_{j, H_{0}}=E_{j, \varepsilon_{0}}$ and $\beta_{H_{0}}=\beta_{\varepsilon_{0}}(1 \leqq j \leqq r)$. Then

$$
\begin{gathered}
\sum_{1 \leqq i \leqq r} \partial\left(\gamma\left(z_{i}\right)\right)^{*} E_{i, H_{0}}=\partial(u)^{*} \delta+\beta_{H_{0}} \\
\sup \left|\beta_{H_{0}}\right| \leqq b_{3} \tau\left(H_{0}\right)^{-p}
\end{gathered}
$$

where $b_{3}=6^{p} b_{2}$. Now Supp $E_{i, H_{0}}$ and $\operatorname{Supp} \beta_{H_{0}}$ are both contained in $\operatorname{Supp} \Psi_{\varepsilon_{0}}$. Moreover, $\|H\| \leqq \tau\left(H_{0}\right) / 2$ if $H \in \operatorname{Supp} \Psi_{\varepsilon_{0}}$. Hence if $H-H_{0} \in \operatorname{Supp} \Psi_{\varepsilon_{0}}$, it follows from Lemma 46 that $H \in V_{1}$ and $\tau(H) \geqq \tau\left(H_{0}\right) / 2$. Let $V\left(H_{0}\right)$ be the set of all $H \in V$ such that $\tau(H) \geqq \tau\left(H_{0}\right) / 2$. Then it is clear that $V\left(H_{0}\right) \subset V_{1}$ and

$$
\begin{gathered}
\phi_{f}\left(H_{0} ; \partial(u)\right)=\sum_{1 \leqq i \leqq r} \int_{V\left(H_{0}\right)} \phi_{f}\left(H ; \partial\left(\gamma\left(z_{i}\right)\right)\right) E_{i, H_{0}}\left(H-H_{0}\right) d H \\
-\int_{V\left(H_{0}\right)} \phi_{f}(H) \beta_{H_{0}}\left(H-H_{0}\right) d H .
\end{gathered}
$$

On the other hand it follows from the definition of $V$ (see $\$ 24)$ that we can choose a number $c_{1}>0$ such that

$$
\left|\Delta\left(H_{0} \exp H\right)\right| \geqq c_{1}\left|\pi_{3}(H)\right| \quad(H \in V) .
$$

Let $q_{1}$ be the number of roots in $P_{3}$. Then it follows from our definition of $t_{1}, \cdots, t_{L}$ that

$$
\left|\pi_{3}(H)\right| \geqq \tau(H)^{q_{1}} \quad\left(H \in V_{1}\right) .
$$

Therefore

$$
\left|\Delta\left(h_{0} \exp H\right)\right| \geqq c_{1} \tau(H)^{q_{1}} \quad\left(H \in V_{1}\right)
$$

Hence

$$
\begin{aligned}
\tau\left(H_{0}\right)^{q_{1}} & \left|\int_{V\left(H_{0}\right)} \phi_{f}\left(H ; \partial\left(\gamma\left(z_{i}\right)\right)\right) E_{i, H_{0}}\left(H-H_{0}\right) d H\right| \\
& \leqq c_{2} \int_{V\left(H_{0}\right)}\left|\phi_{z_{i} f}(H) \Delta\left(h_{0} \exp H\right)\right|\left|E_{i, H_{0}}\left(H-H_{0}\right)\right| d H,
\end{aligned}
$$

where $c_{2}=2^{q_{1}} c_{1}^{-1}$. Moreover, since $\left|\Psi_{\varepsilon}\right| \leqq 1$ and $E_{i}$ are continuous functions on $\mathfrak{h}$, it is clear that

$$
\sup _{H \in V\left(H_{0}\right)}\left|E_{i, H_{0}}\left(H-H_{0}\right)\right| \leqq \sup _{\|H\| \leqq 2}\left|E_{i}(H)\right| \leqq c_{3},
$$


where $c_{3}$ is a positive number independent of $H_{0}$ or $i$. Hence

$$
\tau\left(H_{0}\right)^{q_{1}}\left|\int_{V\left(H_{0}\right)} \phi_{f}\left(H ; \partial\left(\gamma\left(z_{i}\right)\right)\right) E_{i, H_{0}}\left(H-H_{0}\right) d H\right| \leqq c_{2} c_{3} v\left(z_{i} f\right) \quad(1 \leqq i \leqq r) .
$$

Similarly since sup $\left|\beta_{H_{0}}\right| \leqq b_{3} \tau\left(H_{0}\right)^{-p}$, we get

$$
\left|\tau\left(H_{0}\right)\right|^{p+q_{1}}\left|\int_{V\left(H_{0}\right)} \phi_{f}(H) \beta_{H_{0}}\left(H-H_{0}\right) d H\right| \leqq c_{2} b_{3} v(f) .
$$

Moreover, $\tau\left(H_{0}\right) \leqq c \leqq 1$. Hence

$$
\tau\left(H_{0}\right)^{p+q_{1}}\left|\phi_{f}\left(H_{0} ; \partial(u)\right)\right| \leqq c_{2} b_{3} v(f)+c_{2} c_{3} \underset{1 \leqq i \leqq r}{\Sigma} v\left(z_{i} f\right)
$$

for $H_{0} \in V_{1} / 2$ and $f \in C_{c}{ }^{\infty}(G)$. Now

$$
\prod_{1 \leqq i \leqq L} t_{i}(H) \leqq c^{L-1} \tau(H) \quad\left(H \in V_{1}\right) .
$$

This is obvious if $L \geqq 1$ and is also true if $L=0$. Therefore the statement of Lemma 48 follows immediately if we take $q=p+q_{1}$.

26. Proof of Lemma 45(18). Now we come to the proof of Lemma 45. If $L=0$, it is an immediate consequence of Lemma 48 . So we may assume that $L \geqq 1$.

By a monomial $T$ we mean a function on $\mathfrak{h}$ of the form $t_{1}{ }^{q_{1}} t_{2}{ }^{q_{2}} \cdots t_{L}{ }^{q_{L}}$, where $q_{1}, \cdots, q_{L}$ are integers $\geqq 0$. The degree of $T$ is the integer $q_{1}+q_{2}+\cdots+q_{L}$ and we denote it by $d^{0} T$. Since $S\left(\mathfrak{h}_{c}\right)$ is a finite module over $I\left(\mathfrak{h}_{c}\right)=\gamma(3)$ (see [4(f), Lemma 11]), it is also a finite module over $\gamma\left(3_{0}\right)$. Hence we can choose $u_{j}$ $(1 \leqq j \leqq r)$ in $S\left(\mathfrak{h}_{c}\right)$ such that $u_{1}=1$ and

$$
S\left(\mathfrak{h}_{c}\right)=\sum_{1 \leqq j \leqq r} \gamma\left(\mathfrak{Z}_{0}\right) u_{j}
$$

We say that a monomial $T$ has property (P) if there exists a number $a=a(T)$ $(0<a \leqq 1)$ and $\sigma \in \mathscr{S}$ such that

$$
\sup _{H \in a V_{1}} T(H)\left|\phi_{f}\left(H ; \partial\left(u_{j}\right)\right)\right| \leqq \sigma(f) \quad(1 \leqq j \leqq r)
$$

for all $f \in C_{c}{ }^{\infty}(G)$. Now suppose $T$ has property (P) and put

$$
\sigma_{T}(f)=\max _{1 \leqq j \leqq r} \sup _{H \in a V_{1}} T(H)\left|\phi_{f}\left(H ; \partial\left(u_{j}\right)\right)\right| \quad\left(f \in C_{c}^{\infty}(G)\right),
$$

where $a=a(T)$. Then it is obvious that $\sigma_{T} \in \mathscr{S}$ and, for a given $u \in S\left(\mathfrak{h}_{c}\right)$, we can select $z_{i} \in \mathcal{Z}_{0}(1 \leqq i \leqq r)$ such that $u=\Sigma_{1 \leqq i \leqq r} \gamma\left(z_{i}\right) u_{i}$. Hence

$$
\phi_{f}(H ; \partial(u))=\sum_{i} \phi_{z_{i} f}\left(H ; \partial\left(u_{i}\right)\right) \quad\left(H \in V^{\prime}, f \in C_{c}^{\infty}(G)\right) .
$$

(18) Cf. [4(g), pp. 208-211]. 
So it is clear that

$$
\sigma_{T, u}(f)=\sup _{H \in a V_{1}} T(H)\left|\phi_{f}(H ; \partial(u))\right| \leqq \sum_{1 \leqq j \leqq r} \sigma_{T}\left(z_{j} f\right)
$$

for all $f \in C_{c}^{\infty}(G)$ and therefore $\sigma_{T, u} \in \mathscr{S}$.

Hence, in order to prove Lemma 45 , it is obviously enough to obtain the following result.

LEMMA 49. The monomial 1 has property (P).

It is clear from Lemma 48 that monomials with property $(\mathrm{P})$ actually do exist. Let $T$ be a monomial with property (P) of the lowest possible degree. We claim that $T=1$. For otherwise suppose $d^{0} T>0$. Then, without loss of generality, we may assume that $T=t_{1}^{q_{1}} t_{2}^{q_{2}} \cdots t_{L}{ }^{q_{L}}$ and $q_{1} \geqq 1$. Put $T_{2}=t_{2}{ }^{q_{2}} \cdots t_{L}{ }^{q_{L}}$ so that $T=t_{1}{ }^{q_{1}} T_{2}$ and $d^{0} T_{2}<d^{0} T$. Let $a=a(T)$ and, for any $f \in C_{c}^{\infty}(G)$, put

$$
\psi_{f, i}(H)=T_{2}(H) \phi_{f}\left(H ; \partial\left(u_{i}\right)\right) \quad\left(H \in \in^{\prime} a V_{1}, 1 \leqq i \leqq r\right) .
$$

We recall that $H_{1}, \cdots, H_{l}$ is a base for $\mathfrak{h}$ over $R$ such that $t_{i}\left(H_{j}\right)=\delta_{i j}(1 \leqq i, j \leqq l)$. Now choose $z_{i j} \in \mathcal{Z}_{0}(1 \leqq i, j \leqq r)$ such that

$$
H_{1} u_{i}=\sum_{1 \leqq j \leqq r} \gamma\left(z_{i j}\right) u_{j} \quad(1 \leqq i \leqq r) .
$$

Then

$$
\partial \psi_{f, i} / \partial t_{1}=\sum_{j} \psi_{z_{i f f}, j}
$$

on $a V_{1}$ and therefore

on $a V_{1}$. Here

$$
\left|t_{1}^{q_{1}}\left(\partial \psi_{f, i} / \partial t_{1}\right)\right| \leqq \sum_{j} \sigma_{T}\left(z_{i j} f\right) \quad\left(f \in C_{c}^{\infty}(G)\right)
$$

$$
\sigma_{T}(f)=\max _{J} \sup _{\boldsymbol{B} \in a V_{1}} T(H)\left|\phi_{f}\left(H ; \partial\left(u_{j}\right)\right)\right| \quad\left(f \in C_{c}{ }^{\infty}(G)\right)
$$

and it is clear that $\sigma_{T} \in \mathscr{S}$ since $T$ has property (P). Put

$$
\sigma(g)=\sum_{1 \leqq i, j \leqq r} \sigma_{T}\left(z_{i j} g\right) \quad\left(g \in C_{c}^{\infty}(G)\right) .
$$

Then $\sigma$ also lies in $\mathscr{S}$.

For any $b>0$, let $W_{b}$ denote the set of all $H \in \mathfrak{h}$ such that $\left|t_{i}(H)\right| \leqq b(1 \leqq i \leqq l)$. Choose $a_{1}\left(0<a_{1} \leqq 1\right)$ so small that $W_{a_{1}} \subset a V$ and $a_{2}\left(0<a_{2} \leqq a_{1}\right)$ such that $a_{2} V \subset W_{a_{1}}$. Suppose $H \in a_{2} V_{1}$. Then $H^{\prime}=H+\left(a_{1}-t_{1}(H)\right) H_{1} \in W_{a_{1}} \subset a V$. Since $t_{i}(H)>0 \quad(1 \leqq i \leqq L)$ and $t_{1}(H) \leqq\|H\|<a_{2} c \leqq a_{2} \leqq a_{1}$, it follows that $t_{i}\left(H^{\prime}\right)>0(1 \leqq i \leqq L)$ and therefore $H^{\prime} \in a V_{1}$. But $a V_{1}$ being convex, the whole line segment joining $H$ to $H^{\prime}$ liesin $a V_{1}$. On the other hand, we have seen above that

$$
\left|\left(\partial \psi_{f, i} / \partial t_{1}\right)\right| \leqq t_{1}{ }^{-q_{1}} \sigma(f) \quad\left(f \in C_{c}{ }^{\infty}(G)\right)
$$



on $a V_{1}$. Hence, by integrating on this line segment, we get

$$
\left|\psi_{f, i}\left(H^{\prime}\right)-\psi_{f, i}(H)\right| \leqq \sigma(f) \int_{t_{1}(H)}^{a_{1}} s^{-q_{1}} d s
$$

since $t_{1}\left(H^{\prime}\right)=a_{1}$. Moreover,

$$
\left|t_{1}\left(H^{\prime}\right)^{q_{1}} \psi_{f, i}\left(H^{\prime}\right)\right|=T\left(H^{\prime}\right)\left|\phi_{f}\left(H^{\prime} ; \partial\left(u_{i}\right)\right)\right| \leqq \sigma_{T}(f) .
$$

Therefore

$$
\left|\psi_{f, i}\left(H^{\prime}\right)\right| \leqq a_{1}^{-q_{1}} \sigma_{T}(f)
$$

This shows that

$$
\left|\psi_{f, i}(H)\right| \leqq a_{1}^{-q_{1}} \sigma_{T}(f)+\sigma(f) \int_{t_{1}(H)}^{a_{1}} s^{-q_{1}} d s
$$

for $H \in a_{2} V_{1}$ and $f \in C_{c}{ }^{\infty}(G)$.

Now first suppose that $q_{1} \geqq 2$. Then

$$
\int_{t_{1}(H)}^{a_{1}} s^{-q_{1}} d s=\left(q_{1}-1\right)^{-1}\left(t_{1}(H)^{1-q_{1}}-a_{1}^{1-q_{1}}\right) .
$$

Hence if $T_{1}=t_{1}{ }^{q_{1}-1} T_{2}$, it is clear that

$$
\left|T_{1}(H) \phi_{f}\left(H ; \partial\left(u_{i}\right)\right)\right| \leqq a_{1}^{-q_{1}} \sigma_{T}(f)+\sigma(f)
$$

for $H \in a_{2} V_{1}, f \in C_{c}{ }^{\infty}(G)$ and $1 \leqq i \leqq r$. This shows that $T_{1}$ has property (P). But since $d^{0} T_{1}=d^{0} T-1<d^{0} T$, this gives a contradiction. So the case $q_{1} \geqq 2$ is impossible.

Hence $q_{1}=1$. Then

$$
\int_{t_{1}(H)}^{a_{1}} s^{-1} d s=\log \left(a_{1} / t_{1}(H)\right)
$$

and therefore

$$
\left|\psi_{f, i}(H)\right| \leqq a_{1}^{-1} \sigma_{T}(f)+\sigma(f) \log \left(a_{1} / t_{1}(H)\right) \quad(1 \leqq i \leqq r)
$$

for $H \in a_{2} V_{1}$ and $f \in C_{c}{ }^{\infty}(G)$. Put

$$
\sigma^{\prime}(g)=\sigma(g)+a_{1}^{-1} \sigma_{T}(g) \quad\left(g \in C_{c}^{\infty}(G)\right) .
$$

Then $\sigma^{\prime} \in \mathscr{S}$ and

$$
\left|\psi_{f, i}\right| \leqq \sigma^{\prime}(f)\left\{1+\log \left(a_{1} / t_{1}\right)\right\}
$$

on $a_{2} V_{1}$. Put

$$
\sigma_{2}(g)=\sum_{1 \leqq i, \leqq r} \sigma^{\prime}\left(z_{i j} g\right) \quad\left(g \in C_{c}^{\infty}(G)\right)
$$


Then $\sigma_{2} \in \mathscr{S}$ and since

$$
\partial \psi_{f, i} / \partial t_{1}=\sum_{j} \psi_{z_{i j} f, j}
$$

we conclude that

$$
\left|\left(\partial \psi_{f, i} / \partial t_{1}\right)\right| \leqq \sigma_{2}(f)\left\{1+\log \left(a_{1} t_{1}^{-1}\right)\right\}
$$

on $a_{2} V_{1}$. Now choose numbers $a_{3}, a_{4}\left(0<a_{4} \leqq a_{3} \leqq a_{2}\right)$ such that $W_{a_{3}} \subset a_{2} V$ and $a_{4} V \subset W_{a_{3}}$. For any $H \in a_{4} V_{1}$, define

$$
H^{n}=H+\left(a_{3}-t_{1}\left(H_{1}\right)\right) H_{1} .
$$

Then $H^{\prime \prime} \in a_{2} V_{1}$ and so again by integrating along the line segment joining $H$ and $H^{\prime \prime}$, we conclude that

$$
\begin{aligned}
\left|\psi_{f, i}\left(H^{\prime \prime}\right)-\psi_{f, i}(H)\right| & \leqq \sigma_{2}(f) \int_{t_{1}(H)}^{a_{3}}\left(1+\log \left(a_{1} s^{-1}\right)\right) d s \\
& \leqq b \sigma_{2}(f)
\end{aligned}
$$

for $H \in a_{4} V_{1}, f \in C_{c}{ }^{\infty}(G)$ and $1 \leqq i \leqq r$. Here

$$
b=\int_{0}^{a_{3}}\left(1+\log \left(a_{1} s^{-1}\right)\right) d s<\infty .
$$

Now $t_{1}\left(H^{\prime \prime}\right)=a_{3}$ and

$$
t_{1}\left(H^{\prime \prime}\right)\left|\psi_{f, i}\left(H^{\prime \prime}\right)\right| \leqq \sigma_{T}(f)
$$

since $T=t_{1} T_{2}$. Therefore

$$
\left|\psi_{f, i}(H)\right| \leqq a_{3}{ }^{-1} \sigma_{T}(f)+b \sigma_{2}(f) \quad(1 \leqq i \leqq r)
$$

for $H \in a_{4} V_{1}$ and $f \in C_{c}{ }^{\infty}(G)$. This shows that $T_{2}$ has property (P) and therefore again, since $d^{0} T_{2}=d^{0} T-1<d^{0} T$, we get a contradiction. This proves Lemma 49 and hence also Lemma 45.

27. Proof of Theorem 3 in the general case. Now we come to the general case and use of the notation of $\S 16$. Let ${ }_{0} M$ be the centralizer of $\mathfrak{h} \cap \mathfrak{p}$ in $G$ and $M$ the connected component of 1 in ${ }_{0} M$. Then $A \subset{ }_{0} M$ and, by Lemma 30, $M$ is acceptable.

Let $G_{c}$ be a complexification of $G$ and define $j$ as in $\S 18$. Put

$$
\Phi_{0}=j(K) \cap \exp \left((-1)^{1 / 2}(\mathfrak{h} \cap \mathfrak{p})\right) .
$$

LEMMA 50. $\Phi_{0}$ is a finite group. Let $\Phi$ be a finite subset of $G$ such that $j(\Phi)=\Phi_{0}$. Then $A=\Phi A^{0} Z$, where $A^{0}$ is the connected component of 1 in $A$.

Since $\mathfrak{h} \cap \mathfrak{p} \subset \mathfrak{g}_{1}$, we may obviously assume, for the proof of this lemma, that $g$ is semisimple and $G_{c}$ is simply connected. Then $j(K)$ is compact and 
$\Phi_{0} \subset j(A) \cap j(K)=j\left(A_{K}\right)$. Extend $\theta$ to a complex-analytic automorphism of $G_{c}$. Then since $\theta=-1$ on $\mathfrak{p}$, it is clear that $a^{2}=1$ for every $a \in \Phi_{0}$. Therefore since $j\left(A_{K}\right)$ is a compact abelian group, it follows that $\Phi_{0}$ is finite.

Now $A=A_{K} A_{\mathfrak{p}}$ and $A_{\mathfrak{p}} \subset A^{0}$ (see Corollary 4 of Lemma 26). Hence, in order to prove the second statement, it would be enough to verify that $j\left(A_{K}\right)=\Phi_{0} j\left(A_{K}{ }^{0}\right)$, where $A_{K}{ }^{0}$ is the analytic subgroup of $G$ corresponding to $\mathfrak{h} \cap \mathfrak{f}$.

Let $A_{c}$ be the Cartan subgroup of $G_{c}$ corresponding to $\mathfrak{h}_{c}$. Put $\mathfrak{u}=\mathfrak{f}+(-1)^{1 / 2} \mathfrak{p}$ and and define $U$ and $\eta$ as in $\S 17$. Then if $a \in j\left(A_{K}\right)$, it is clear that $a \in U \cap A_{c}=\exp \left(\mathfrak{h}_{c} \cap \mathfrak{u}\right)$. But $\mathfrak{h}_{c} \cap \mathfrak{u}=\mathfrak{h} \cap \mathfrak{F}+(-1)^{1 / 2}(\mathfrak{h} \cap \mathfrak{p})$ and therefore $a=a_{1} a_{2}$, where $a_{1} \in j\left(A_{K}{ }^{0}\right)$ and $a_{2} \in \Phi_{0}$. This proves that $j\left(A_{K}\right)=\Phi_{0} j\left(A_{K}{ }^{0}\right)$.

Lemma 51. Let $a \in \Phi$ and $m \in M$. Then $a$ and $m$ commute.

Since $M$ is connected, this follows from the fact that its Lie algebra m commutes with $\mathfrak{h} \cap \mathfrak{p}$.

Fix an order in the space of (real-valued) linear functions $\lambda$ on $\mathfrak{h} \cap \mathfrak{p}$ and, for any such $\lambda$, let $\mathfrak{g}_{\lambda}$ denote the space of all $X \in \mathfrak{g}$ such that $[H, X]=\lambda(H) X$ for all $H \in \mathfrak{h} \cap \mathfrak{p}$. Put $\mathfrak{n}=\Sigma_{\lambda>0} \mathfrak{g}_{\lambda}$. Then $\mathfrak{n}$ is a nilpotent subalgebra of $\mathfrak{g}$. Let $N$ be the analytic subgroup of $G$ corresponding to $n$. It is clear that ${ }_{0} M$ normalizes $n$. Put

$$
d(m)=\left|\operatorname{det}(\operatorname{Ad}(m))_{\mathfrak{n}}\right|^{1 / 2} \quad\left(m \in_{0} M\right),
$$

where the subscript $\mathfrak{n}$ denotes restriction on $\mathfrak{n}$. Put $G_{0}=\operatorname{Ad}(G)$ and let $K_{0}$ denote the image of $K$ in $G_{0}$ under the homomorphism $x \rightarrow \operatorname{Ad}(x)$. Then $K_{0}$ is compact. For any $x \in G$ and $y_{0} \in G_{0}$, define $x^{y_{0}}=y x y^{-1}$ where $y$ is any element of $G$ such that $y_{0}=\operatorname{Ad}(y)$. Put

$$
\bar{f}(x)=\int_{K_{0}} f\left(x^{k_{0}}\right) d k_{0}, \quad g_{f}(m)=d(m) \int_{N} \bar{f}(m n) d n
$$

for $f \in C_{c}{ }^{\infty}(G), x \in G$ and $m \in_{0} M$. Here $d k_{0}$ and $d n$ are the Haar measures on $K_{0}$ and $N$, respectively, and $\int_{K_{0}} d k_{0}=1$.

Introduce an order on the space of real-valued linear functions on $(-1)^{1 / 2}(\mathfrak{h} \cap \mathfrak{t})+\mathfrak{h} \cap \mathfrak{p}$ which is compatible (see [4(g), p. 195]) with the one already chosen above. We may assume, without loss of generality, that the set $P$ of positive roots of $(\mathfrak{g}, \mathfrak{h})$ is defined with respect to this order. Since every root of $(\mathfrak{m}, \mathfrak{h})$ is imaginary, it follows from Corollary 5 of Lemma 26 that $A \cap M=A^{0}$. Let $m \rightarrow m^{*}$ denote the natural projection of $M$ on $M^{*}=M / A^{0}$ and define

$$
\begin{array}{cc}
F_{g}{ }^{M}(h)=\Delta_{M}(h) \int_{M^{*}} g\left(h^{m^{*}}\right) d m^{*} & \left(h \in A^{0} \cap M^{\prime}\right), \\
v_{M}(g)=\int_{A^{0}}\left|\Delta_{M}(h) F_{g}{ }^{M}(h)\right| d h & \left(g \in C_{c}^{\infty}(M)\right),
\end{array}
$$


where $d m^{*}$ is the invariant measure on $M^{*}$ and $M^{\prime}$ is the set of those elements of $M$ which are regular in $M$.

Let $\mathfrak{M}$ be the subalgebra of $\left(\mathfrak{G}\right.$ generated by $\left(1, m_{c}\right)$ and $\mathfrak{Z}_{M}$ the center of $\mathfrak{M}$. Then we have the isomorphism $\mu=\mu_{\mathfrak{g} / \mathbf{m}}$ of 3 into $\mathcal{Z}_{M}$ (see $\$ 12$ ). Moreover, $G=K M N$ from $[4(\mathrm{~g})$, Lemma 11$]$.

LEMMA 52. For any $a \in \Phi, p u t$

$$
g_{f, a}(m)=g_{f}(a m) \quad\left(m \in M, f \in C_{c}{ }^{\infty}(G)\right) .
$$

Then $g_{f, a} \in C_{c}{ }^{\infty}(M)$ and

$$
g_{z f, a}=\mu(z) g_{f, a} \quad(z \in \mathcal{3}) .
$$

Moreover, if $d m^{*}$ and $d n$ are suitably normalized, we have the relation

$$
F_{f}(a h)=\xi_{\rho}(a) F_{g_{f, .}}{ }^{M}(h)
$$

for $f \in C_{c}^{\infty}(G), h \in A^{0} \cap\left(a^{-1} G^{\prime}\right)$ and $a \in \Phi$.

Although the proof of this lemma is not difficult, it is rather long. Hence we postpone it to another paper.

We can now complete the proof of Theorem 3. It is clear that

$$
\Delta_{M}(a h)=\xi_{\rho}(a) \Delta_{M}(h) \quad(a \in \Phi, h \in A) .
$$

Hence we conclude from Lemma 52 that

$$
v_{M}\left(g_{f, a}\right)=\int_{A^{0}}\left|\Delta_{M}(h) F_{f}(a h)\right| d h \leqq v(f) .
$$

We have seen (Lemma 30$)$ that $M$ is acceptable and every root of $(\mathfrak{m}, \mathfrak{h})$ is imaginary. Moreover, $Z_{M}$ is a finite module over $\mu\left(3_{0}\right)$ by Lemma 21 . Hence Theorem 3 holds for $\left(M, A^{0}, \mu\left(Z_{0}\right), v_{M}\right)$ in place of $\left(G, A, 3_{0}, v\right)$. Therefore for any $u \in \mathfrak{S}\left(\mathfrak{h}_{c}\right)$, we can, in view of Lemma 52 , choose a finite set of elements $z_{1}, \cdots, z_{r} \in 3_{0}$ such that

$$
\sup _{h \in A^{\prime}}\left|F_{f}(h ; u)\right| \leqq \max _{a \in \Phi} \sum_{1 \leqq i \leqq r} v_{M}\left(\mu\left(z_{i}\right) g_{f, a}\right) \leqq \sum_{1 \leqq i \leqq r} v\left(z_{i} f\right)
$$

for $f \in C_{c}{ }^{\infty}(G)$. This proves Theorem 3 .

28. The local summability of $|D|^{-1 / 2}$. Let $l=\operatorname{rank} G$ and put $D=D_{l}$ in the notation of $\S 3$. Then $D$ is an analytic function on $G$ and

$$
D(h)=\operatorname{det}(1-\operatorname{Ad}(h))_{g / h}=(-1)^{p} \Delta(h)^{2} \quad(h \in A),
$$

where $p$ is the number of positive roots of $(\mathfrak{g}, \mathfrak{h})$.

LEMMA 53. $|D|^{-1 / 2}$ is locally summable on $G$. 
Let $C$ be a compact subset of $G$. Then we can choose $f \in C_{c}^{\infty}(G)$ such that $f \geqq 0$ everywhere and $f \geqq 1$ on $C$. Then it is clear that

$$
\int_{C}|D|^{-1 / 2} d x \leqq \int_{G}|D|^{-1 / 2} f d x
$$

Let $A_{i}(1 \leqq i \leqq r)$ be a maximal set of Cartan subgroups of $G$ no two of which are conjugate under $G$. Put

$$
G_{i}=\bigcup_{x \in G} x A_{i}^{\prime} x^{-1},
$$

where $A_{i}{ }^{\prime}=A_{i} \cap G^{\prime}$. Then $G^{\prime}$ is the disjoint union of $G_{1}, \cdots, G_{r}$ (see [4(e), Lemma 5]). Hence it would be enough to verify that

$$
\int_{G_{i}}|D|^{-1 / 2} f d x<\infty \quad(1 \leqq i \leqq r) .
$$

So fix $i$ and put $A=A_{i}$. Then $G_{i}=G_{A}$ in the notation of $\S 23$ and it follows from Lemma 41 and $[4(\mathrm{~h})$, Theorem 2$]$ that

$$
\int_{G_{A}}|D|^{-1 / 2} f d x=c \int_{A}\left|F_{f}(h)\right| d h<\infty
$$

This proves the lemma.

29. Appendix. Put $\rho(x)=\left(x_{1}{ }^{2}+x_{2}{ }^{2}+\cdots+x_{n}{ }^{2}\right)^{1 / 2} \geqq 0$ for $x \in R^{n}$.

LEMMA 54. Let $\alpha$ be a real number and $D=\partial^{k} / \partial x_{i_{1}} \partial x_{i_{2}} \cdots \partial x_{i_{k}}$. Then

$$
\begin{aligned}
D \rho^{\alpha} & =\sum_{0 \leqq j \leqq k} p_{j} \rho^{\alpha-j-k}, \\
D\left(\rho^{\alpha} \log \rho\right) & =\sum_{0 \leqq j \leqq k} P_{j} \rho^{\alpha-j-k}+(\log \rho) \sum_{0 \leqq j \leqq k} Q_{j} \rho^{\alpha-j-k},
\end{aligned}
$$

where $p_{j}, P_{j}$ and $Q_{j}$ are homogeneous polynomials in $\left(x_{1}, \cdots, x_{n}\right)$ of degree $j$.

This follows by an easy induction on $k$.

Corollary 1. If $\alpha>k$, then $\rho^{\alpha}$ and $\rho^{\alpha} \log \rho$ are functions of class $C^{k}$ on $\boldsymbol{R}^{n}$.

This is obvious from the lemma.

COROLlaRY 2. $\rho^{k-1} D \rho$ remains bounded on $R^{n}$.

We know that

$$
\rho^{k-1} D \rho=\sum_{0 \leqq j \leqq k} p_{j} \rho^{-j},
$$

where $p_{j}$ is a homogeneous polynomial of degree $j$ in $\left(x_{1}, \cdots, x_{n}\right)$. Our assertion therefore follows from the obvious fact that $\left|p_{j}\right| \rho^{-j}$ is bounded on $\boldsymbol{R}^{n}$. 
The following lemma is implicit in the paper of Morrey and Nirenberg [6, p. 281].

LEMMA 55. Let $h$ be a function in $C^{\infty}(R)$ which is constant on the intervals $(-\infty, 0]$ and $[1,+\infty)$. Choose two numbers $r, \delta$ such that $0<\delta \leqq r \leqq 1$ and put

$$
H_{r, \delta}(x)=h\left(\delta^{-1}(\rho(x)-r)\right) \quad\left(x \in R^{n}\right) .
$$

Then for each integer $k \geqq 0$, there exists a number $c_{k} \geqq 0$, independent of $r$ and $\delta$, such that

$$
\left|D H_{r, \delta}\right| \leqq c_{k} \delta^{-k}
$$

for $D=\partial^{k} / \partial x_{i_{1}} \partial x_{i_{2}} \cdots \partial x_{i_{k}}\left(1 \leqq i_{1}, \cdots, i_{k} \leqq n\right)$.

We use induction on $k$. If $k=0$, we can take $c_{0}=\sup |h|$. So let us assume that $k \geqq 1$ and put $h^{\prime}(t)=d h / d t(t \in R)$. Then $h^{\prime}$ also satisfies the conditions of the lemma and

$$
\partial H_{r, \delta} / \partial x_{i}=\delta^{-1} H_{r, \delta^{\prime}} \cdot \partial \rho / \partial x_{i} \quad(1 \leqq i \leqq n),
$$

where

$$
H_{r, \delta^{\prime}}(x)=h^{\prime}\left(\delta^{-1}(\rho(x)-r)\right) \quad\left(x \in R^{n}\right) .
$$

Now $H_{r, \delta}(x)=0$ unless $r \leqq \rho(x) \leqq r+\delta$ and therefore

$$
\sup _{x}\left|D H_{r, \delta}\right| \leqq \delta^{-1} \sup _{\rho \geqq \delta}\left|D^{\prime}\left(H_{r, \delta^{\prime}} \cdot \partial \rho / \partial x_{i_{k}}\right)\right|,
$$

where $D^{\prime}=\partial^{k-1} / \partial x_{i_{1}} \cdots \partial x_{i_{k-1}}$. Hence if we expand

$$
D^{\prime}\left(H_{r, \delta} \cdot \partial \rho / \partial x_{i_{k}}\right)
$$

by means of the Leibniz formula, make use of Corollary 2 of Lemma 54 and apply the induction hypothesis to $H_{r, \delta}$, we get the required assertion.

Put $\Delta=\Sigma_{1 \leqq i \leqq n}\left(\partial / \partial x_{i}\right)^{2}$ and let $\delta$ denote the Dirac measure concentrated at the origin.

LeMMA 56. If $n$ is odd

and if $n$ is even

$$
\Delta^{l+(n-1) / 2} \rho^{2 l-1}=c_{l} \delta \quad(l \geqq 1)
$$

$$
\Delta^{l+n / 2}\left(\rho^{2 l} \log \rho\right)=c_{l}^{\prime} \delta \quad(l \geqq 0) .
$$

Here $c_{l}$ and $c_{l}^{\prime}$ are nonzero numbers and the above relations are meant in the sense of the theory of distributions.

This is well known (see [7, p. 47]).

LEMmA 57. Fix integers $d \geqq 0$ and $r \geqq 1$. Then we can choose an integer $m \geqq 1$ and a function e on $R^{n}$ of class $C^{2 m(r-1)+d}$ such that 


$$
\Delta^{m r} e=\delta .
$$

Choose $m$ so large that $2 m>d+n$. First suppose $n$ is odd. Then $l=m r-(n-1) / 2$ is an integer and

$$
2 l=2 m r-n+1>d+1 .
$$

Hence $l \geqq 1$. Put $e=c_{l}^{-1} \rho^{2 l-1}$. Then

$$
\Delta^{m r} e=\Delta^{l+(n-1) / 2} e=\delta
$$

and $2 l-1=2 m r-n=2 m(r-1)+2 m-n>2 m(r-1)+d$. Hence $e$ is of class $C^{2 m(r-1)+d}$ by Corollary 1 of Lemma 54 .

On the other hand if $n$ is even put $l=m r-n / 2$. Then $2 l=2 m r-n>d$ and therefore $l$ is positive. Now put

$$
e=\left(c_{l}{ }^{\prime}\right)^{-1} \rho^{2 l} \log \rho .
$$

Then $2 l=2 m r-n>2 m(r-1)+d$ and therefore again $e$ is of class $C^{2 m(r-1)+d}$.

\section{REFERENCES}

1. A. Borel, Groupes linéaires algébriques, Ann. of Math. (2) 64 (1956), 20-82.

2. A. Borel and G. D. Mostow, On semi-simple automorphisms of Lie algebras, Ann. of Math. (2) 61 (1955), 389-405.

3. N. Bourbaki, Groupes et algèbres de Lie, Chapitre I, Algèbres de Lie, Hermann, Paris, 1960.

4. (a) Harish-Chandra, On representation of Lie algebras, Ann. of Math. (2) 50 (1949), 900-915.

(b) - Representations of a semisimple Lie group on a Banach space. I, Trans. Amer. Math. Soc. 75 (1953), 185-243.

(c) - The Plancherel formula for complex semisimple Lie groups, Trans. Amer. Math. Soc. 76 (1954), 485-528. 564-628.

(d) - Representations of semisimple Lie groups. VI, Amer. J. Math. 78 (1956), 98-163.

(e) - The characters of semisimple Lie groups, Trans. Amer. Math. Soc. 83 (1956), $87-120$.

(f) - Differential operators on a semisimple Lie algebra, Amer. J. Math. 79(1957), 193-257.

(g) - Fourier transforms on a semisimple Lie algebra. I, Amer. J. Math. 79 (1957), 653-686.

$\left(\mathrm{g}_{2}\right)$ _ Fourier transforms on a semisimple Lie algebra. II, Amer. J. Math. 79 (1957),

(h) —-, A formula for semisimple Lie groups, Amer. J. Math. 79 (1957), 733-760.

(i) _- Spherical functions on a semisimple Lie group. I, Amer. J. Math. 80 (1958) 241-310.

(j) - Invariant eigendistributions on semisimple Lie groups, Bull. Amer. Math. Soc. 69 (1963), 117-123. 
(k) —, Invariant distributions on Lie algebras, Amer. J. Math. 86 (1964), 271-309.

(1) - Invariant differential operators and distributions on a semisimple Lie algebra, Amer. J. Math. 86 (1964), 534-564.

(m) - Some results on an invariant integral on a semi-simple Lie algebra, Ann. of Math. (2) 80 (1964), 551-593.

(n) —_, Invariant eigendistributions on a semisimple Lie algebra, Inst. Hautes Études Sci. Publ. Math. No. 27.

5. S. Helgason, Differential geometry and symmetric spaces, Academic Press, New York, 1962.

6. C. B. Morrey and L. Nirenberg, On the analyticity of solutions of linear elliptic systems of partial differential equations, Comm. Pure Appl. Math. 10 (1957), 271-290.

7. L. Schwartz, Théorie des distributions. I, Hermann, Paris, 1950.

8. H. Weyl, The structure and representation of continuous groups, The Institute for Advanced Study, Princeton, N. J., 1935.

The Institute for Advanced Study,

Princeton, NeW Jersey 